\title{
Psychological and Physiological Effects of Light and Colour on Space Ulsers
}

$\mathcal{A}$ Dissertation Submitted in $\mathcal{F}$ ulfillment of the Requirements for the Degree of Master of Engineering

by

Nadeen Ab6as

School of Electrical and Computer Engineering

Science, Engineering and Technology Portfolio

RMIT University

March 2006 
(C) 2006 Nadeen Abbas

All Rights Reserved 


\section{Declaration}

I certify that except where due acknowledgement has been made, the work is that of the author alone; the work has not been submitted previously, in whole or in part, to qualify for any other academic award; the content of the thesis is the result of work which has been carried out since the official commencement date of the approved research program; and, any editorial work, paid or unpaid, carried out by a third party is acknowledged.

Signed:

Date:

$\mathcal{N a d e e n ~ A 6 6 a s ~}$ 
This dissertation is dedicated to my Parents, Family 8 Friends 


\section{Acknowledgements}

I would like to first thank Almighty God for all his blessings, one of them is being able to complete this work which I hope will contribute to the welfare of people.

I would like at this juncture to express my deepest appreciation and gratitude to my supervisor A/Prof. Dinesh Kank Kumar for his support, assistance and advice throughout my candidature at RMIT University. My deepest thanks are extended to my co-supervisor Dr. Neil Mclachlan.

I gratefully acknowledge the financial support from the Australian Postgraduate Awards (APA), which grated me a scholarship for supporting my research and living expenses.

I also wish to express my appreciation to A/Prof. Panlop Zeephongsekul, Faculty of Applied Science Mathematics and Statistics at RMIT University, for his help and valuable comments on statistical analysis. I also extend my deepest thanks and appreciation to Dr. SeedAhmed Mahmoud and Dr. Esref Turker, RMIT University for their great support, help and encouragement throughout my candidature.

I would like to thank my colleagues Prashant Suryanarayanan, Zuraini Dahari, Djuwari Djuwari and Alexandru Fechete, with whom I shared the research office, for their support and the wonderful time I spent with them. I would like to particularly thank my colleague Prashant Suryanarayanan for his continuous help, assistance and support.

I would like to express my deepest appreciation and gratitude to my cousins Amin Abbas and Nada Abuhamdeh, my friends Wigdan Mahamed, Yasmeen Hiyabo and Ruqaya Basheer for their unlimited support. They are always there for me and are the ones I fall back upon when I am weak. My deepest gratitude is extended to Mr Abdelkarim Hilli for his great support and 
advice. His doors were always open to me whenever I seek his help.

Finally I would like to dedicate this work to my parents, sister and brothers. I always tried my best to make them proud of me. They have always been there to support me. No words can ever express what they mean to me and how grateful I am to have them. 


\section{Publications}

\section{Conference Publications}

1. Nadeen Abbas, Dinesh Kumar and Neil Mclachlan, The Psychological and Physiological Effects of Light and Colour on Space Users, $27^{\text {th }}$ Annual International Conference of the IEEE Engineering in Medicine and Biology Society, Shanghai, China, September 2005.

2. Nadeen Abbas, Dinesh Kumar and Neil Mclachlan, Measuring the emotional and physiological effects of light and colour on space users, $2^{\text {nd }}$ International Conference on Informatics in Control, Automation \& Robotics, Barcelona, Spain, September 2005.

3. Nadeen Abbas, Prashant Suryanarayanan and Dinesh K. Kumar, The Psychophysiological Impact of Colours and Lighting on Space Users, $5^{\text {th }}$ Australian Space Science Conference, September 2005.

\section{Submitted Journal Publications}

1. Nadeen Abbas, Dinesh K. Kumar and Neil Mclachlan, The Psychological and Physiological Effects of Light and Colour on Space Users, Journal of Color Research and Application .

2. Nadeen Abbas, Prashant Suryanarayanan and Dinesh K. Kumar, Correlation of Psychological and Physiological Measures of Emotion, Journal of Bioengineering 
and Technology. 


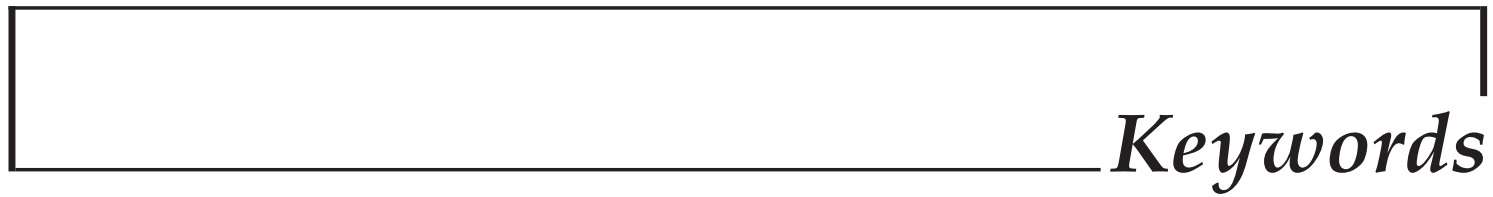

Colour, Electrocardiogram, Emotion, Heart Rate, Heart Rate Variability, Light, Lighting, Self Assessment Manikin, Skin Conductance. 


\section{Preface}

he main topic of this reseach is to study the emotional effects of colour and lighting on

ـ space users using physiological and psychological measures. It is clear from literature that the choices of colour and lighting of constructed spaces are not based on scientific facts, thus there is a need for a research that studies the impact of colour and light on people using subjective and objective measures. I hope that this work will help Architects and Interior-Designers in their choices of colour and light and inspires further research into this field.

Melbourne

March 2006

$\mathcal{N a d e e n ~ A 6 6 a s ~}$ 
Declaration $\quad$ i

Acknowledgements

Publications $\quad$ v

Keywords vii

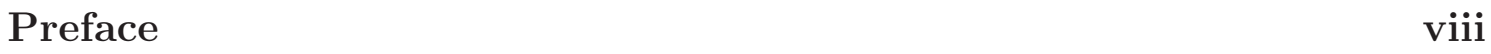

$\begin{array}{ll}\text { Acronyms } & \mathrm{xx}\end{array}$

Abstract $\quad$ xxi

1 Introduction 1

2 Literature Review 3

2.1 Light, colour and emotion . . . . . . . . . . . . . . . . 3

2.2 Measuring emotion . . . . . . . . . . . . . . . 5

2.3 Physiological measures $\ldots \ldots \ldots \ldots$

2.3.1 Cardiac Activity . . . . . . . . . . . . . . . . . 7

2.3.2 Skin conductance . . . . . . . . . . . . . . . . 8

2.4 Psychological measure: Self Assessment Manikin . . . . . . . . . . . . . . . 8

2.5 Aim of the research $\ldots \ldots \ldots \ldots \ldots$ 
3 Methodology 10

3.1 Participants selection $\ldots \ldots \ldots \ldots$

3.2 Experimental set up . . . . . . . . . . . . . . . . . . . . . . . . . 11

3.3 Equipments and signal processing . . . . . . . . . . . . . . . . . 13

3.4 Statistical analysis . . . . . . . . . . . . . . . . . . . . . . . . . . . . . 13

4 Results and Observation $\quad 15$

4.1 ECG results and analysis . . . . . . . . . . . . . . . . . . . . . . . 15

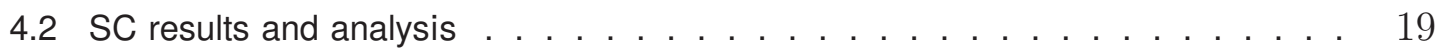

4.3 SAM . . . . . . . . . . . . . . . . . . . . . . 22

4.3 .1 Arousal . . . . . . . . . . . . . . . . . . . . . . . 23

4.3 .2 Valence . . . . . . . . . . . . . . . . . . 25

4.4 One-way Analysis of Variance (ANOVA) . . . . . . . . . . . . . . . 27

4.5 Two-way Analysis of Variance (ANOVA) . . . . . . . . . . . . . . . . . 29

5 Discussion $\quad 31$

5.1 Summary of Discussion . . . . . . . . . . . . . . . . 36

6 Conclusion $\quad 39$

A ECG Recordings $\quad 41$

$\begin{array}{ll}\text { B SC Recordings } & 72\end{array}$

$\begin{array}{ll}\text { C HR Charts } & 78\end{array}$

$\begin{array}{lr}\text { D SC Charts } & 84\end{array}$

$\begin{array}{lr}\text { E Arousal Charts } & 90\end{array}$

$\begin{array}{lr}\text { F Valence Charts } & 96\end{array}$

$\begin{array}{ll}\text { G Plain Language Summary } & 102\end{array}$

$\begin{array}{ll}\text { H Participant Consent Form } & 104\end{array}$

$\begin{array}{ll}\text { Bibliography } & 105\end{array}$ 
3.1 Electrode Placement for ECG Recordings . . . . . . . . . . . . . . . . . . . . 13

3.2 Electrode Placement for SC Recordings . . . . . . . . . . . . . . . . . . 13

4.1 ECG signal for participant 1 under White light . . . . . . . . . . . . . . . . 16

4.2 Difference in mean HR for subject $5 \ldots \ldots \ldots$

4.3 SC signal for participant $10 \ldots \ldots \ldots$

4.4 Change in mean SC for participant $10 \ldots \ldots . \ldots . \ldots 22$

4.5 Change in arousal participant $3 \ldots \ldots \ldots$

4.6 Change in valence participant $3 \ldots \ldots \ldots \ldots$

A.1 Participant 1 - ECG Recordings for participant 1 for all colour lights . . . . . . . 42

(a) White light . . . . . . . . . . . . . . . . . . 42

(b) Blue-low light . . . . . . . . . . . . . . . . . . . . . . . . . . . 42

(c) Blue-high light . . . . . . . . . . . . . . . . . . . . . . . 42

(d) Green-low light . . . . . . . . . . . . . . . . . . . . . . . . . . . . . 42

(e) Green-high light . . . . . . . . . . . . . . . . . . . . 43

(f) Red-low light . . . . . . . . . . . . . . . . . . . . . . . . . . 43

(g) Red-hig light . . . . . . . . . . . . . . . . . . . . . . 43

(h) Natural light . . . . . . . . . . . . . . . . . . . . . . 43

A.2 Participant 2 - ECG Recordings for participant 2 for all colour lights . . . . . . . 44

(a) White light . . . . . . . . . . . . . . . . . . 44 
(b) Blue-low light . . . . . . . . . . . . . . . . . . . . . . . . . 44

(c) Blue-high light . . . . . . . . . . . . . . . . . . . . 44

(d) Green-low light . . . . . . . . . . . . . . . . . . . . . . . . . . . . 44

(e) Green-high light . . . . . . . . . . . . . . . . . . . 45

(f) Red-low light . . . . . . . . . . . . . . . . . . . . . . . . . . . . . . . . 45

(g) Red-hig light . . . . . . . . . . . . . . . . . . . . . . . 45

(h) Natural light . . . . . . . . . . . . . . . . . . . . 45

A.3 Participant 3 - ECG Recordings for participant 3 for all colour lights . . . . . . . 46

(a) White light . . . . . . . . . . . . . . . . . . 46

(b) Blue-low light . . . . . . . . . . . . . . . . . . . . 46

(c) Blue-high light . . . . . . . . . . . . . . . . . . 46

(d) Green-low light . . . . . . . . . . . . . . . . . . . . . . . . . 46

(e) Green-high light . . . . . . . . . . . . . . . . . 47

(f) Red-low light . . . . . . . . . . . . . . . . . . . . . . . . . 47

(g) Red-hig light . . . . . . . . . . . . . . . . . . . . . 47

(h) Natural light . . . . . . . . . . . . . . . . . . . 47

A.4 Participant 4 - ECG Recordings for participant 4 for all colour lights . . . . . . . 48

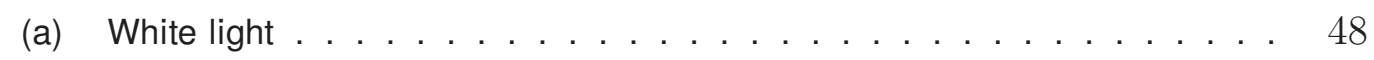
(b) Blue-low light . . . . . . . . . . . . . . . . . . . . . . . . . . . . 48
(c) Blue-high light . . . . . . . . . . . . . . . . . . . . . 48
(d) Green-low light . . . . . . . . . . . . . . . . . . . . . . . . . . . 48
(e) Green-high light . . . . . . . . . . . . . . . . . . . . . . . 49
(f) Red-low light . . . . . . . . . . . . . . . . . . . . . . . . . . . . . . . . . 49
(g) Red-hig light . . . . . . . . . . . . . . . . . . . . . . . . . . . 49
(h) Natural light . . . . . . . . . . . . . . . . . . . . . . . . . . . . . . . 49

A.5 Participant 5 - ECG Recordings for participant 5 for all colour lights . . . . . . 50
(a) White light . . . . . . . . . . . . . . . . 50
(b) Blue-low light . . . . . . . . . . . . . . . . . . . . 50
(c) Blue-high light . . . . . . . . . . . . . . . . . . . 50
(d) Green-low light . . . . . . . . . . . . . . . . . . . 50
(e) Green-high light . . . . . . . . . . . . . . . . . 51 
(f) Red-low light . . . . . . . . . . . . . . . . . . . . . . . 51

(g) Red-hig light . . . . . . . . . . . . . . . . . . . . . . . 51

(h) Natural light . . . . . . . . . . . . . . . . . . 51

A.6 Participant 6 - ECG Recordings for participant 6 for all colour lights . . . . . . . 52

(a) White light . . . . . . . . . . . . . . . . . 52

(b) Blue-low light . . . . . . . . . . . . . . . . . . . . . . 52

(c) Blue-high light . . . . . . . . . . . . . . . . . . . . . . 52

(d) Green-low light . . . . . . . . . . . . . . . . . . . . . . . . . . . . . 52

(e) Green-high light . . . . . . . . . . . . . . . . . . . 53

(f) Red-low light . . . . . . . . . . . . . . . . . . . . . . . . . . . 53

(g) Red-hig light . . . . . . . . . . . . . . . . . . . . . 53

(h) Natural light . . . . . . . . . . . . . . . . . . 53

A.7 Participant 7 - ECG Recordings for participant 7 for all colour lights . . . . . . . 54

(a) White light . . . . . . . . . . . . . . . . . 54

(b) Blue-low light . . . . . . . . . . . . . . . . . . . . . . . . 54

(c) Blue-high light . . . . . . . . . . . . . . . . . . . . 54

(d) Green-low light . . . . . . . . . . . . . . . . . . . . . . . . . 54

(e) Green-high light . . . . . . . . . . . . . . . . . 55

(f) Red-low light . . . . . . . . . . . . . . . . . . . . . . . . 55

(g) Red-hig light . . . . . . . . . . . . . . . . . . . . 55

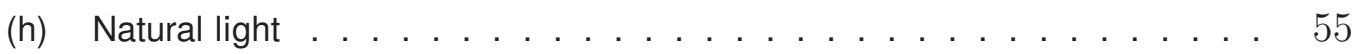

A.8 Participant 8 - ECG Recordings for participant 8 for all colour lights . . . . . . . 56

(a) White light . . . . . . . . . . . . . . . . 56

(b) Blue-low light $\ldots \ldots \ldots \ldots \ldots$

(c) Blue-high light . . . . . . . . . . . . . . . . . 56

(d) Green-low light . . . . . . . . . . . . . . . . . . . . . . . 56

(e) Green-high light . . . . . . . . . . . . . . . . . . . 57

(f) Red-low light . . . . . . . . . . . . . . . . . . . 57

(g) Red-hig light . . . . . . . . . . . . . . . . . . . . . 57

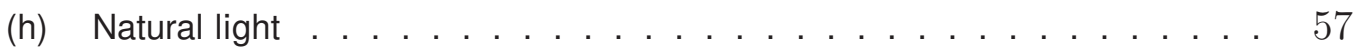

A.9 Participant 9 - ECG Recordings for participant 9 for all colour lights . . . . . . 58 


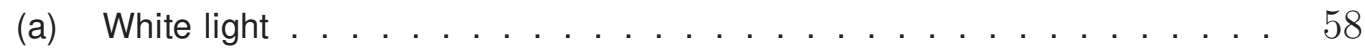

(b) Blue-low light $\ldots \ldots \ldots \ldots$. . . . . . . . . . . . . . . . . 58

(c) Blue-high light . . . . . . . . . . . . . . . . . . . . . . . 58

(d) Green-low light . . . . . . . . . . . . . . . . . . . . . 58

(e) Green-high light . . . . . . . . . . . . . . . . . . . . . . . 59

(f) $\quad$ Red-low light . . . . . . . . . . . . . . . . . . . . . . . . . . . . 59

(g) Red-hig light . . . . . . . . . . . . . . . . . . . . . . . . . 59

(h) Natural light . . . . . . . . . . . . . . . . . . . . . . . . 59

A.10 Participant 10 - ECG Recordings for participant 10 for all colour lights . . . . . 60

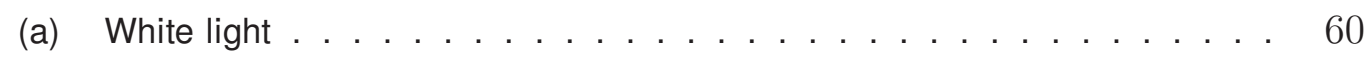

(b) Blue-low light . . . . . . . . . . . . . . . . . 60 60

(c) Blue-high light . . . . . . . . . . . . . . . . . . . 6 60

(d) Green-low light . . . . . . . . . . . . . . . . . . . . . . . 60

(e) Green-high light . . . . . . . . . . . . . . . . . 61

(f) Red-low light . . . . . . . . . . . . . . . . . . . . . . 61

(g) Red-hig light . . . . . . . . . . . . . . . . . . . . . . . . 61

(h) Natural light . . . . . . . . . . . . . . . . . . 61

A.11 Participant 11 - ECG Recordings for participant 11 for all colour lights . . . . . 62
(a) White light . . . . . . . . . . . . . . . . . . 62
(b) Blue-low light . . . . . . . . . . . . . . . . . . . . . . . 62
(c) Blue-high light . . . . . . . . . . . . . . . . . . 62
(d) Green-low light . . . . . . . . . . . . . . . . . . . . . . . . . . . 62
(e) Green-high light . . . . . . . . . . . . . . . . . . . 63

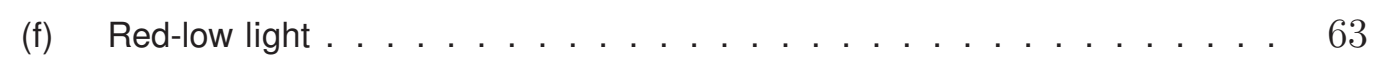
(g) Red-hig light . . . . . . . . . . . . . . . . . . . . . . . 63

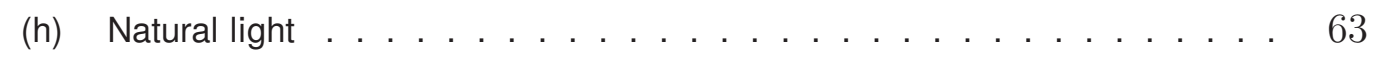

A.12 Participant 12 - ECG Recordings for participant 12 for all colour lights . . . . . 64
(a) White light . . . . . . . . . . . . . . . . . . 64
(b) Blue-low light . . . . . . . . . . . . . . . . . . . . . . . . . . 64
(c) Blue-high light . . . . . . . . . . . . . . . . . . . . . 64
(d) Green-low light . . . . . . . . . . . . . . . . . . . . . . . . . . . 64 
(e) Green-high light . . . . . . . . . . . . . . . . 6 65

(f) Red-low light . . . . . . . . . . . . . . . . . . . . . 65

(g) Red-hig light . . . . . . . . . . . . . . . . . . . . . . . 65

(h) Natural light . . . . . . . . . . . . . . . . . 65

A.13 Participant 13 - ECG Recordings for participant 13 for all colour lights . . . . . 66
(a) White light . . . . . . . . . . . . . . . . 66
(b) Blue-low light . . . . . . . . . . . . . . . . . . 66
(c) Blue-high light . . . . . . . . . . . . . . . . . . . . 66
(d) Green-low light . . . . . . . . . . . . . . . . . . . . . 66
(e) Green-high light . . . . . . . . . . . . . . . 67
(f) Red-low light . . . . . . . . . . . . . . . . . . . . . . . 67
(g) Red-hig light . . . . . . . . . . . . . . . . . . . . . 67
(h) Natural light . . . . . . . . . . . . . . . . . 6 6 67

A.14 Participant 14 - ECG Recordings for participant 14 for all colour lights . . . . . 68

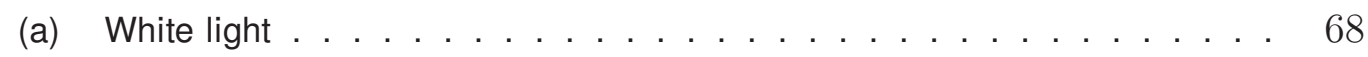
(b) Blue-low light . . . . . . . . . . . . . . . . . . . . . . . . 68
(c) Blue-high light . . . . . . . . . . . . . . . . . . . 68
(d) Green-low light . . . . . . . . . . . . . . . . . . . . . . . 68
(e) Green-high light . . . . . . . . . . . . . . . . . . . . . . . . . . . . 69
(f) Red-low light . . . . . . . . . . . . . . . . . . . . . . . . . 69
(g) Red-hig light . . . . . . . . . . . . . . . . . . . . . 69
(h) Natural light . . . . . . . . . . . . . . . . . . . 69

A.15 Participant 15 - ECG Recordings for participant 15 for all colour lights . . . . . 70

(a) White light . . . . . . . . . . . . . . . . . 70

(b) Blue-low light . . . . . . . . . . . . . . . . . . . 70

(c) Blue-high light . . . . . . . . . . . . . . . . . . 70

(d) Green-low light . . . . . . . . . . . . . . . . . . . . 70

(e) Green-high light . . . . . . . . . . . . . . . . . 71

(f) Red-low light . . . . . . . . . . . . . . . . . . . . . . . . . 71

(g) Red-hig light . . . . . . . . . . . . . . . . . . 71

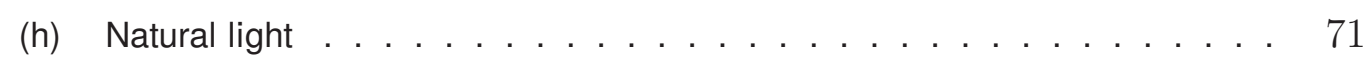


(a) Participant $1 \ldots \ldots \ldots \ldots \ldots$. . . . . . . . . . . . . . . . . . . .

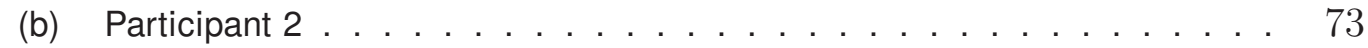

(c) Participant $3 \ldots \ldots \ldots \ldots$. . . . . . . . . . . . . . . . . . . . . . . . . .

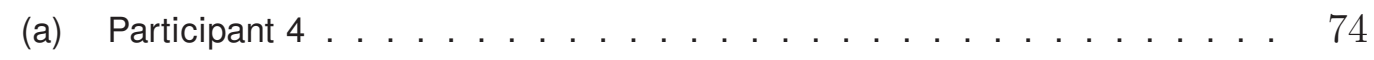

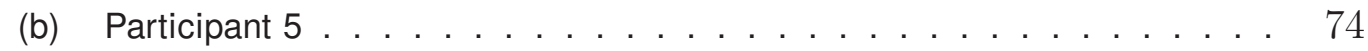

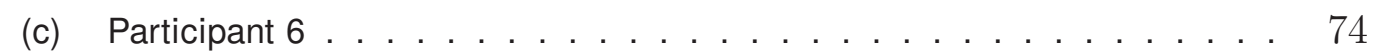

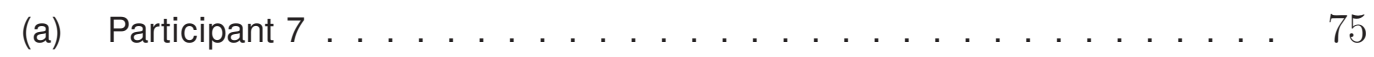

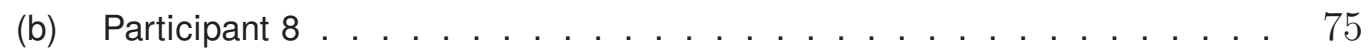

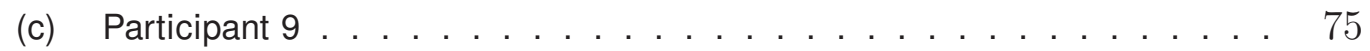

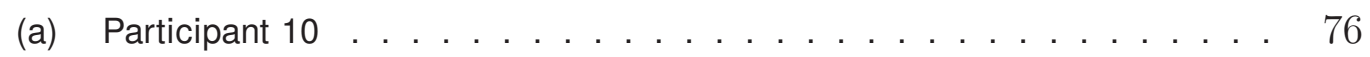

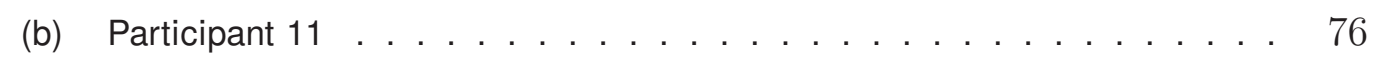

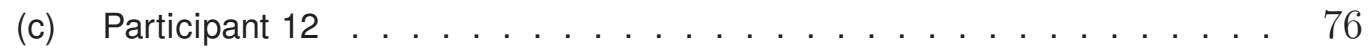

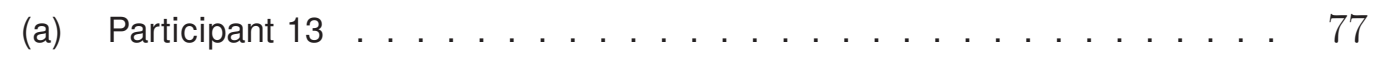

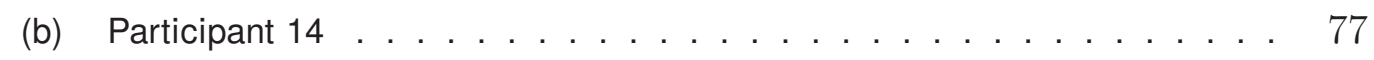

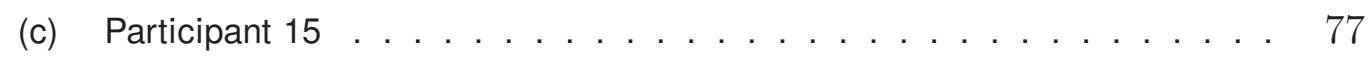

(a) Participant $1 \ldots \ldots \ldots \ldots \ldots$

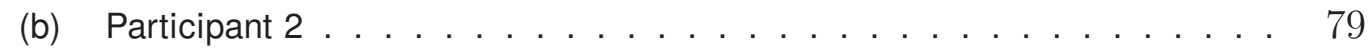

(c) Participant $3 \ldots \ldots \ldots \ldots \ldots$

(a) Participant $4 \ldots \ldots \ldots \ldots$. . . . . . . . . . . . . . . . . . 80

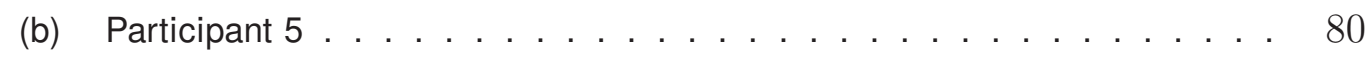

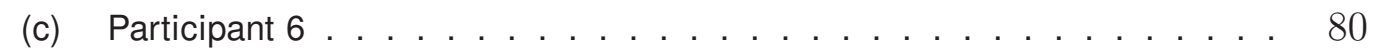

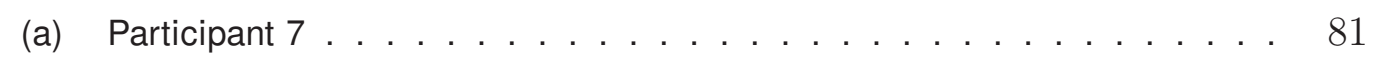

(b) Participant $8 \ldots \ldots \ldots \ldots$. . . . . . . . . . . . . . . . . . . 81

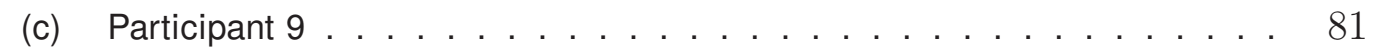

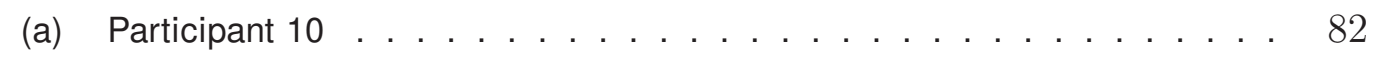

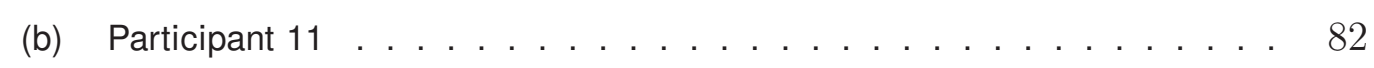

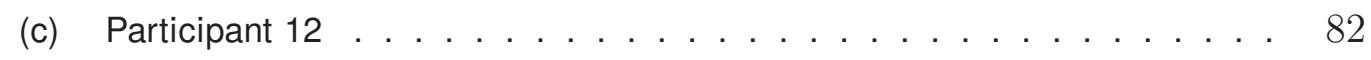

(a) Participant $13 \ldots \ldots \ldots \ldots$. . . . . . . . . . . . . . . . . . 83

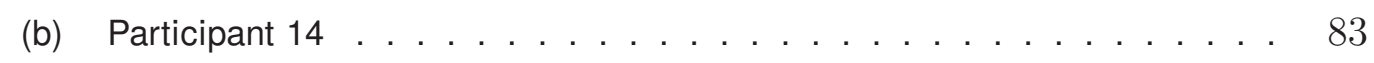

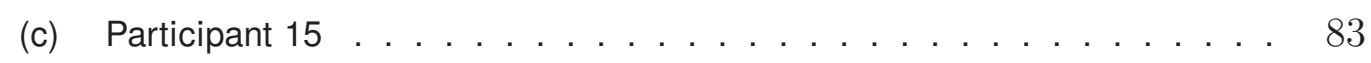




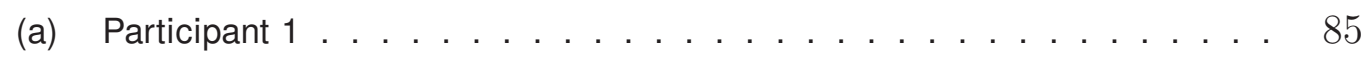

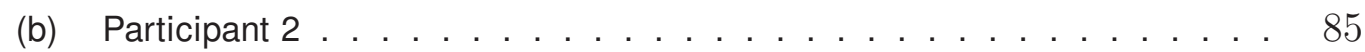

(c) Participant $3 \ldots \ldots \ldots \ldots \ldots$. . . . . . . . . . . . . . . . . . . . . 85

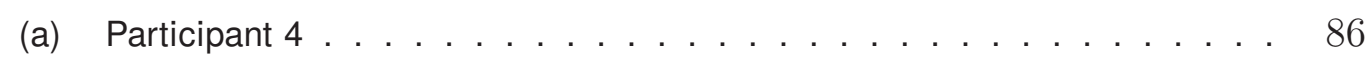

(b) Participant $5 \ldots \ldots \ldots \ldots$

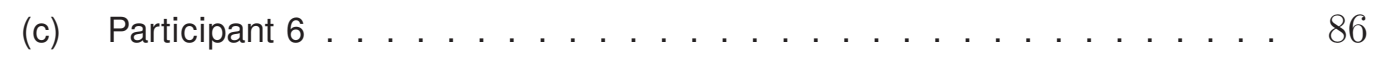

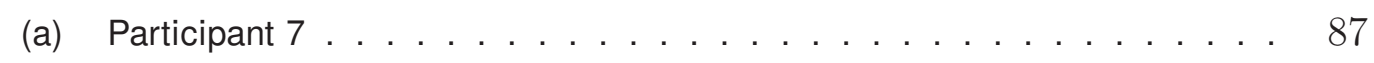

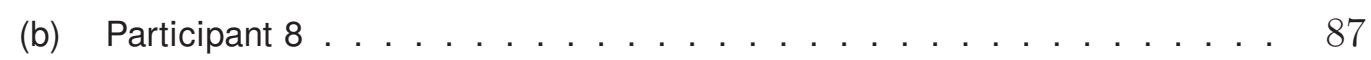

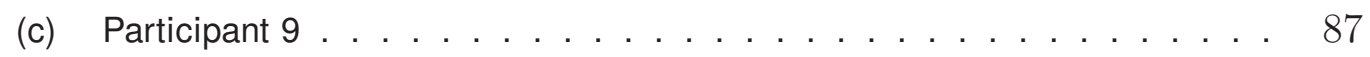

(a) Participant $10 \ldots \ldots \ldots \ldots$

(b) Participant $11 \ldots \ldots \ldots \ldots \ldots$

(c) Participant $12 \ldots \ldots \ldots \ldots$. . . . . . . . . . . . . . . . . . . 88

(a) Participant $13 \ldots \ldots \ldots \ldots \ldots$

(b) Participant $14 \ldots \ldots \ldots \ldots$

(c) Participant $15 \ldots \ldots \ldots \ldots$

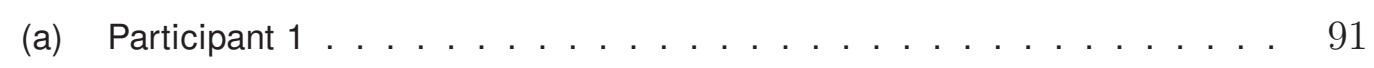

(b) Participant $2 \ldots \ldots \ldots \ldots 1 \ldots \ldots \ldots \ldots$

(c) Participant $3 \ldots \ldots \ldots \ldots$. . . . . . . . . . . . . . . . . . . 91

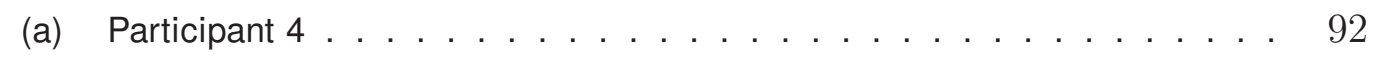

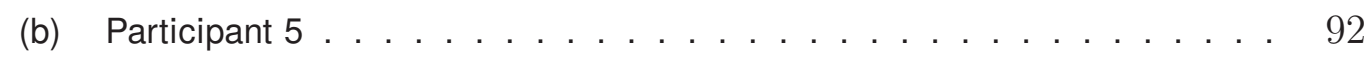

(c) Participant $6 \ldots \ldots \ldots$. . . . . . . . . . . . . . . . . . . . . 92

(a) Participant $7 \ldots \ldots \ldots \ldots \ldots$

(b) Participant $8 \ldots \ldots \ldots \ldots$

(c) Participant $9 \ldots \ldots \ldots \ldots \ldots$

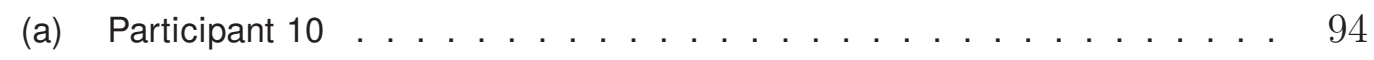

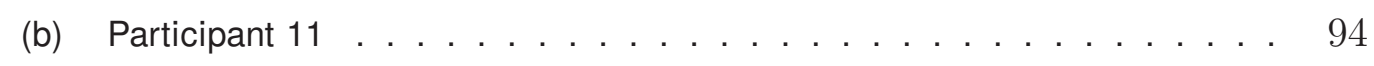

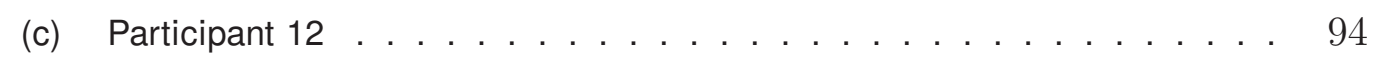

(a) Participant $13 \ldots \ldots \ldots \ldots \ldots$

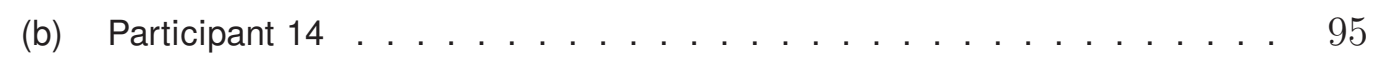

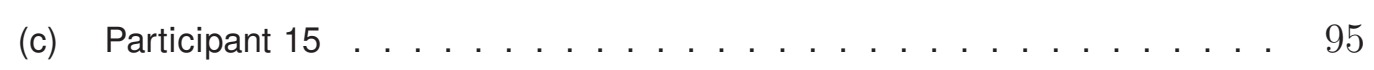




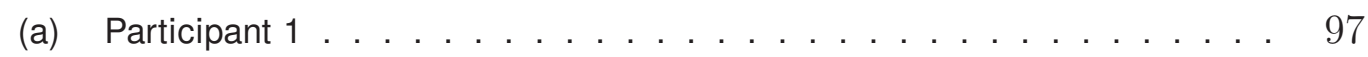

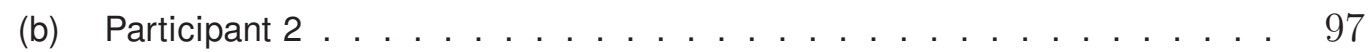

(c) Participant $3 \ldots \ldots \ldots 7 \ldots$

(a) Participant $4 \ldots \ldots \ldots \ldots$

(b) $\quad$ Participant $5 \ldots \ldots \ldots$

(c) Participant $6 \ldots \ldots \ldots \ldots$

(a) Participant $7 \ldots \ldots \ldots \ldots 9 . \ldots \ldots \ldots$

(b) Participant $8 \ldots \ldots \ldots 9 . \ldots \ldots$

(c) Participant $9 \ldots \ldots \ldots$

(a) Participant $10 \ldots \ldots \ldots$. . . . . . . . . . . . . . . . . . . . . . . .

(b) Participant $11 \ldots \ldots \ldots \ldots$. . . . . . . . . . . . . . . . . . . . .

(c) Participant $12 \ldots \ldots \ldots \ldots$. . . . . . . . . . . . . . . . . . . . . . . . . . .

(a) Participant $13 \ldots \ldots \ldots 10 \ldots$

(b) Participant $14 \ldots \ldots \ldots \ldots 1 \ldots \ldots \ldots \ldots$

(c) Participant $15 \ldots \ldots \ldots \ldots$. . . . . . . . . . . . . . . . . . . . . . . 
3.1 Intensities of coloured lights . . . . . . . . . . . . . . . . . . . . . . 12

4.1 RR \& HR values for participant $10 \ldots \ldots \ldots$

4.2 Mean HR (beats/min) $\ldots \ldots \ldots \ldots$

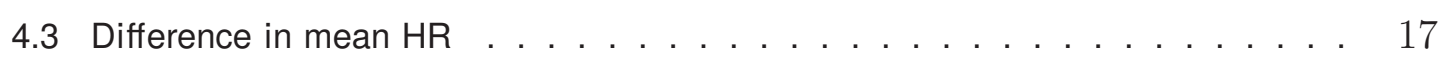

4.4 Normalised values of mean HR (White light as reference) . . . . . . . . . . . 18

4.5 Mean SC values . . . . . . . . . . . . . . . . . . . . . . . . . . . . . . 20

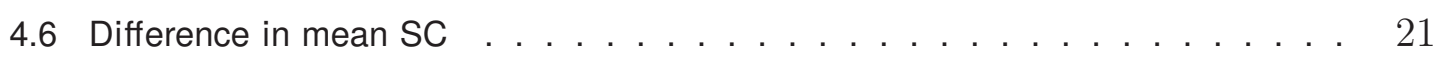

4.7 Normalised values of mean SC (white light as reference) . . . . . . . . . . 21

4.8 Values of arousal rating . . . . . . . . . . . . . . . . . . 23

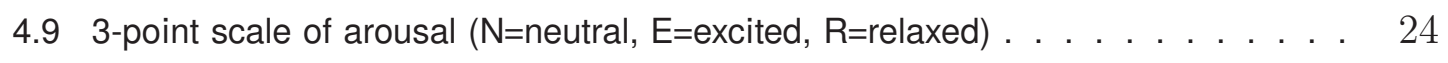

4.10 Values of valence rating . . . . . . . . . . . . . . . . . . 25

4.11 3-point scale of valence ( $\mathrm{N}=$ neutral, $\mathrm{H}=$ happy, $\mathrm{UH}=$ unhappy). . . . . . . . . . 27

4.12 One-way ANOVA for HR . . . . . . . . . . . . . . . . . . . . . . 28

4.13 One-way ANOVA for SC . . . . . . . . . . . . . . . . . . . . . . 28

4.14 One-way ANOVA for arousal . . . . . . . . . . . . . . . . . . . . . . 29

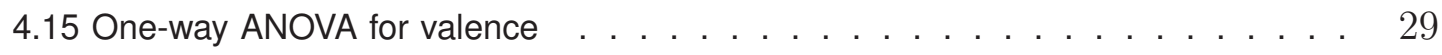

4.16 Two-way ANOVA for $\mathrm{HR} \ldots \ldots \ldots$. . . . . . . . . . . . . . . . . 30

4.17 Two-way ANOVA for SC . . . . . . . . . . . . . . . . . . . 30

4.18 Two-way ANOVA for arousal . . . . . . . . . . . . . . . . . . 30

4.19 Two-way ANOVA for valence . . . . . . . . . . . . . . . . . . . . . 30 


\title{
Acronyms
}

\author{
ANS - Autonomic Nervous System \\ ANOVA - Analysis of Variance \\ ECG - Electrocardiogram \\ EDA - Electrodermal Activity \\ HR - Heart Rate \\ HRV - Heart Rate Variability \\ SC - Skin Conductance \\ SAM - Self Assessment Manikin \\ SS - Sum of Squares \\ df - Degrees of Freedom \\ MS - Mean Squares
}


7 he impact of colour and lighting conditions on the emotions and performance of people $\perp$ is gaining greater importance in our urban societies. While large resources are allocated for well designed spaces with the right choices of colour and lighting conditions, there is little scientific evidence that supports these choices.

Although the literature on light and colour is extensive, it does not present a uniform set of findings for a consistent perspective on the influence of colour and light. Most of the research reported in this field uses subjective measures to study the emotional effects of light and colour on people. It has been reported in literature that emotion manifests itself in three separate sections; (i) physiological (i.e. objective measures), (ii) psychological (subjective measures), (iii) behavioral, and it is necessary that studies not be limited to the assessment of a single response but include sample measures from each of the three manifestations.

This research is built on the current body of knowledge that there is a need for a study on the emotional effects of light and colour on people using physiological and psychological measures, to ensure the objectivity and reproductivity of the experiments.

It is well documented in literature that there is close correlation between emotions, heart rate (HR), and skin conductance (SC). Hence HR and SC are expected to be good physiological measures of environmental conditions on people. Thus this thesis reports changes in the HR, SC and self-assessment reports of arousal and valence (SAM) for people when exposed to different colour and intensity lights. The aim is to help provide an objective rationale for the choice for light intensity and colour by architects, interior designers and other professionals. 
The experiments were conducted on 15 participants who were exposed to 8 different colour and intensity light conditions. The participants' HR and SC were recorded under each colour and intensity light, and they were asked to complete SAM.

The research demonstrates that there is a change in $\mathrm{HR}, \mathrm{SC}$, arousal and valence of participants due to change in the colour and intensity of lights. However, the direction of change was subject dependent, where the same colour and intensity light can have different effects on people. The research suggests that architects and designers of any space must take into account the individual differences of the predicted users when designing the lights and colours.

It is also seen from the results that some colour and intensity lights have greater impact on the emotions of participants than others. Although it is not possible to correlate the colour and lighting conditions to a specific effect on all participants, general effects for some colour lights were drawn from the results.

It is well documented in literature that $\mathrm{HR}$ and $\mathrm{SC}$ are a good measure of emotion. However the results of this study show very high inter subject variation in HR and SC. This is due to people having different HR and SC in normal conditions. This research demonstrates that the use of HR and SC to measure the effect of a stimulus on a group of people is unreliable because it is hard to compare the results. 
CHAPTER 1

\section{Introduction}

he impact of colour and lighting conditions on the emotions and performance of space
users is gaining greater importance in our urban societies. While large resources are allocated for well designed spaces with the right choices of colour and lighting conditions, there is little scientific evidence that supports these choices. Although the literature on light and colour is extensive, it does not present a uniform set of findings for a consistent perspective on the influence of colour and light.

Most research that reports the emotional effects of light and colour on people are based on subjective measures, however the outcome of subjective tests is questionable. It has been reported in literature that emotion manifests itself in three separate sections (i) physiological (i.e. objective measures), (ii) psychological (i.e. subjective measures) and (iii) behavioural, and it is necessary that studies not be limited to the assessment of a single response.

Another problem is that researchers in this field have used different scales, which make the comparison between the studies almost impossible.

Research on the physiological and psychological effects of colour was reported in literature; however the research reported studies the short term effects of colour on people where they are exposed to the colours for 6 seconds [1]. The results of the research are useful to study the effects of colours in advertising however they can not be used for architecture and design.

It is seen from literature that the choices of light and colour by architects and designers are not based on scientific facts. Therefore there is a need for a study on the emotional effects 
of light and colour on people using physiological and psychological measures, to ensure the objectivity and reproductivity of the experiments.

It is well documented in literature that there is close correlation between emotions, heart rate (HR) and skin conductance (SC) [2], [3], [4], [5], [6], [7], [8], [9]. Hence HR and SC are expected to be good physiological measures of environmental conditions on people. Thus this thesis reports changes in the HR, SC and self-assessment reports of arousal and valence (SAM) for people when exposed to different colour and intensity lights. The aim is to help provide an objective rational for the choice for light intensity and colour by architects, interior designers and other professionals.

The thesis is divided into six chapters. Chapter 1 contains the introduction. Chapter 2 contains the literature review, which reports the literature around the topic of the research and discusses how the research is related to it. Chapter 3 describes the methodology, which reports the methods followed to conduct the experiments. Chapter 4 reports the results and observations, which presents the results of the experiments. A sample recording, arbitrarily chosen, is presented in this chapter while all recordings are presented in the appendix. Chapter 5 contains the discussion, which lists the observations reported from the previous chapter providing a discussion for each observation. Chapter 6 contains the conclusion which gives a summary of the research. 
CHAPTER 2

\section{Literature Review}

7 his chapter discusses the literature around the topic of this research and how the re-

L search is related to it. There are four main aspects in this research; the first is the emotional effects of light and colour on people. The second aspect is measuring emotions, which requires physiological and psychological measures. The second aspect leads to the third and fourth aspects, which are the physiological measures and the psychological measures used in the research.

\subsection{Light, colour and emotion}

There are a number of factors in a constructed environment that influence the psychological and emotional state of the users. Light intensity and colour are two important factors with impact on the users performance in the space. While people are known to adapt to different environments, it is believed that there is a loss of productivity and general well being if they are not in certain conditions

It was noted by Illumination Engineering Society of North America (IES) that lighting can play an important role in reinforcing special perception, activity and mood setting [10]. Flagge reports that light should produce a mood and atmosphere in a room, which corresponds to people's demand and expectation [11]. Turner also reports that changes in the intensity of a light source can also alter the perceptual mood of a space [12]. 
There are a number of publications that report research conducted to determine the effect of various lighting and colour conditions on peoples' emotions. Most of these are based on subjective measures, where participants complete questionnaires to describe their feelings after being exposed to different lighting and colours conditions.

Research conducted by Frasca on interior design for ambulatory care facilities reports some important design factors. The research reports that colour and lighting have a profound influence on patients and consumers, such as consumer satisfaction, stress level, health and well being. The paper also reports that the effects of colour and lighting are inseparable and lighting intensity affects the perception of colour [13].

Research by Knez et.al. report subjective tests to study the effects of the recommended office lighting on mood and cognitive performance. The work demonstrates that the physical setting of an office and the use of artificial light can have a significant impact on the mood of the inhabitants [14]. Another research conducted by Hathaway found that students who study under daylight like light were less absent and achieved higher scores than those working under yellowish-orange sodium vapor bulbs [15]. Hupka et.al. conducted a research on a study of word association and found that individuals readily associated colour with adjectives of emotion. A number of researches done on trade and sales suggest that improving light quality could improve productivity [16], [17], [18].

The literature on light and colour is extensive, however it does not present a uniform set of findings for a consistent perspective on the influence of colour and light. Veitch et.al. report in their research "one problem facing designers and engineers is that there is no commonlyaccepted metric of lighting quality that predicts the effects of the luminous environment on the occupants. It is commonly assumed that poor lighting quality has negative impact on the ability of people to perform their work; however, few studies have attempted to quantify lighting quality as a whole, and none of these has attempted to relate quantified quality to task performance" [19]. They also report that comparison between studies is nearly impossible because different researches have used different scales. Also their documentation of the outcome measures fall far short of the detail normally demanded by behavioural scientists, which creates problems for later re-evaluation of the data [19].

Another problem is that most of the reported research is based on subjective tests; however the outcome of subjective tests is questionable. It has been reported that subjective tests such 
as questionnaires are insufficient and may be misleading especially when conducted at the end of the experiment [20], [21]. Often, participants may mix their emotions between the start and the end of experiments, and the recollection of a series of emotions that occurred earlier may lack accuracy. Tiller also reports that examination of a multi-faceted concept such as lighting quality should not rest on a single measurement nor a single measurement technique [22].

Veitch et.al. report that " to assess lighting quality requires accurate measurements of the relevant human activities, as well as accurately specified, appropriate lighting conditions. Lighting research has typically failed to attend to the response side of the equation, possibly because many researchers approach lighting from a background on the technical (stimulus) side". They also report that past attempts to develop a metric for lighting quality, even in the limited case of office lighting, have largely failed. One important reason for this failure is poor science: poor research design, statistical analysis and reporting [23].

Valdez et.al. also report that "it is difficult to abstract general patterns of findings from the color-emotion literature, because experiments have used highly limited assessments of reactions to color or have used differenct and non overlapping dimentions to assess emotional reaction to color". He reports "A comprehensive system for the description of emotions is needed to compare and contrast findings from studies that have used these nonoverlapping dependent measures" [24].

Thus there is a need for a research that studies the effect of light and colour on people using a number of measurements. The research data must also have the details that allow later re-evaluation of it.

\subsection{Measuring emotion}

Thus there is a need for a research that studies the emotional effects of light and colour, it is important to study how to measure emotions.

Lang et.al. report that emotion manifests itself in three separate sections; (i) physiological, (ii) psychological (i.e. subjective experience) and (iii) behavioral. The physiological is the change in the physical properties of the person, psychological being the subjective experience while the behavioral is manifest by the physical actions such as approach and avoidance, each of these associated with specific measures [20], [21]. They also report that it is necessary that 
studies not be limited to the assessment of a single response but include sample measures from each of the three manifestations.

Based on that it can be seen that the designers' choices of colour and light are not based on scientific facts as many of the research done in this area is based only on psychological measures. Birren also discusses that light and color undoubtedly affect body functions just as they exert an influence over so called mind and emotion. He said that feelings of dissatisfaction and discomfort are derived in the luminous environment where biological information has been lacking [25].

Binjamin et.al. studied the impact of short film clips on people. Each of these clips was on for 6 seconds, some of them were coloured and some were black and white. Skin conductance, heart rate and facial movement were used in the research as physiological measures in addition to subjective tests to study the short-term effect of colours on people. The results indicated the influence of colour in the subjective reports of emotional experience however no significant influence was reported in the physiological measures. The paper recommends the further studies to explore the impact of colour and light on heart rate of the people using stimuli of longer time durations [1].

The authors are unable to find any research that has measured the physiological changes in people due to light and colour stimuli of long duration. To identify the best choice of interior conditions for buildings such as colour and light, there is a need to determine the physiological changes in people under these conditions when the exposure is long enough to allow the participants of the study to adapt to them.

Birren also discusses that the failure of many lighting designs result from putting the decorative needs first and hoping that the practical needs will be satisfied as a byproduct in much the same way as designing a teapot for its looks and then being surprised when it does not pour [26].

The aim of this research is to provide an answer for the choice of light and colour conditions in a constructed environment, which is for the betterment of health and well being of the occupants. This study reports experimental research conducted to identify the psychological and physiological changes in participants when exposed to different colour and lighting conditions for periods of time that allow them to adapt to these conditions. The physiological measures used are heart rate and skin conductance, the psychological measure is Self Assessment Manikin. 


\subsection{Physiological measures}

Physiological and psychological measures were used as a measure of emotions in this research. The physiological measures used were cardiac activity and skin conductance.

\subsubsection{Cardiac Activity}

Cardiac activity is closely linked with the emotional state of an individual. Electrocardiograph (ECG) is a non-invasive recording of the electrical activity of the heart; it is usually recorded from the surface of the body. It is a good indicator for health of the heart and for measuring heart rate $(\mathrm{HR}) . \mathrm{HR}$ is defined as the number of heart beats per minute and is recognized as a non-invasive means to assess the state of the Autonomic Nervous System (ANS) [27]. Increased activity of the sympathetic branch of the ANS causes an increase in the HR while increase in para-sympathetic branch causes a slow down in HR. When the individual is under stress, the sympathetic activity is more profound than the para-sympathetic activity causing an imbalance in the ANS and hence the HR of the person increases [28].

It is well documented there is a close correlation between HR and emotions. Levenson et.al. report that anger, fear and sadness are associated with significant changes to the heart rate [2]. Vrana et.al. also report that there is a change in heart rate due to emotion. The acceleration of heart rate is greater during disgust, joy and anger imageries than during pleasant imageries [3] or neutral imagery [4]. Smith et.al. report that the heart rate measure offered strong support between anticipated effort and arousal [5].

It was also reported by Steiner et.al. that changes in attention and emotional processing have been associated with changes in heart rate [29]. Ardle ed.al. report that an individuals heart rate is a good measure of physical stress [30].

Heart rate variability (HRV) analysis is a measure of the variability in heart rate, specifically variability in intervals between successive $R$ waves- $R R$ intervals. $R R$ interval is defined as time in milliseconds between two normal $R$ to $R$ waves of an ECG wave. The HRV is also a dynamic signal whose statistical properties vary with time [31]. 


\subsubsection{Skin conductance}

Skin conductance (SC) is one of the fastest responding non-invasive measures of ANS activity. It is a method of capturing the autonomic nerve response as a parameter of the sweat gland. Physically, SC is a change in the electrical properties of the skin in response to different kinds of stimuli. The response is measured by the change in voltage from the surface of the skin [32].

The close correlation between SC and emotions is well documented in the literature. Lane et.al. report that SC shows different values for different emotions when studying the effects of emotional valence, arousal and attention on neural activation during visual processing of pictures [6]. Storm et.al. also report that SC fluctuations are a measure of preoperative stress [7]. Healy et.al. used SC as a measure of the emotional stress developed during driving [8]. Heo et.al. used SC as a measure of emotional response to web advertising [9].

It is reported in literature that SC response is a more reliable measure for determining ANS activity that the tonic measures [33], [34], [35]. The magnitude of SC is related to the magnitude of the stimulus, in other words stronger stimuli elicit stronger responses [34].

\subsection{Psychological measure: Self Assessment Manikin}

The psychological measure used for measuring emotions is the Self Assessment Manikin (SAM). SAM is a picture-oriented instrument devised by Lang to directly assess the pleasure, arousal and dominance associated in response to an object or event. These measures were used because they provide a descriptive system for emotions. Mehrabian et.al. report that the dimensions of pleasure, arousal and dominance could provide a general description oe emotions [36]. They also report that most of the reliable variance in 42 verbal-report scales could be accounted for terms of the PAD (i.e., pleasure, arousal and dominace) emotion scales [24].

SAM was originally implemented as an interactive computer program, and later was expanded to include a paper-and -pencil version for use in groups and mass screening. SAM ranges from a smiling happy figure to a frowning, unhappy figure when representing the valence dimension, and ranges from an exciting, wide-eyed figure to a relaxed, sleepy figure for the arousal dimension. The dominance dimension represents changes in control with changes in the size of SAM [37], [38]. However in this research only the dimensions of valence and arousal in SAM were used. 
Lang et.al. report that SAM has been used effectively to measure emotional responses in a variety of situations, including reactions to pictures [39], [40], images [41], advertisements [42], painful stimuli [43] and more. They also report that SAM is a useful instrument when determining the subjective experience of emotion associated with processing most stimuli, and can be employed with variety of subject populations, including non-English speaking subjects, children, people with language disorder and all clinical syndromes[37].

\subsection{Aim of the research}

Based on literature, there is a need for a study on the emotional effects of light and colour on people using physiological and psychological measures, to ensure the objectivity and reproductivity of the experiments. This research reports changes in the HR, SC and SAM for people when exposed to different colour and intensity lights for periods of time that allowed them to adapt to the conditions. The aim is to help provide an objective rational for the choice for light intensity and colour by architects, interior designers and other professionals. 
CHAPTER 3

Methodology

7 his research is built on the current body of knowledge that physiological and psycho-

ـ logical measures are required to determine the emotional effects of light and colour on the users of a constructed environment. The research reports changes in heart rate (HR), skin conductance (SC) and Self Assessment Manikin (SAM) due to changes in the colour and intensity of lights. In order to do that, controlled experiments were conducted with all possible variables that may affect the measures, except colour and light intensity, kept constant. This was necessary to ensure that the changes recorded were due to the effect of colour and lighting conditions only.

The data was then analysed and descriptive statistical evaluations (such as mean and standard deviation) have been used to examine the effect of light and colour on the HR, SC and SAM. Analysis of variance (ANOVA) was then performed to determine the statistical significance in the changes detected.

\subsection{Participants selection}

The experiments were conducted on 15 healthy participants comprising 3 males and 12 females. They were not under any medication that may affect their mental and neural activities. All participants had normal colour vision. The participants were recruited with the help of posters and most were university students. Their participation was voluntary. Ethics approval for the 
experiments was obtained from RMIT University Human Ethics committee, and participants completed consent forms before the experiment. Participants were made aware of the details of the experiments and were also informed that they could stop the experiment if they chose to.

\subsection{Experimental set up}

The experiments were conducted under controlled conditions. Attempts were made to keep all variables, other than colour and intensity of light, constant. This was necessary to ensure that any psychological or physiological changes detected are due to change in the colour and intensity of lights.

Environmental conditions such as air temperature, humidity, furniture and layout were kept constant. All experiments were conducted in a quite laboratory early in the morning to exclude noise disturbance and circadian rhythms as potential confounds. It was important to conduct all experiments at the same time of the day. Scheer et.al. report that their research "demonstrated that light increases resting HR depending on the phase of the day-night cycle and the intensity of light" [44]. Leproult et.al. also report that their research "'demonstrate an effect of the corticotropic axis that is dependent on time of the day" [45].

The order and interval of exposure to lights were also kept constant for all participants. The duration of the experiment was approximately 110 minutes, during this time the participants were engaged in a prepared non-controversial discussion on the history of architecture so as to reduce mental stress due to boredom. The style of speaking was maintained constant to avoid the sound and conversation related variations during the experiments.

The experiments were conducted in a neutrally coloured and furnished multi user laboratory approximately $10 \mathrm{~m} \times 4 \mathrm{~m}$ in size. The room had only one window which was covered by a large $1.5 \mathrm{~m} \times 2 \mathrm{~m}$ opaque non-reflecting white screen. The walls were covered with white cardboard. Participants were seated comfortably facing the screen, approximately $1.5 \mathrm{~m}$ away from it. They were exposed to the different light and colour conditions by illuminating the screen with flood lights. They were also exposed to natural light from the window by removing the screen that is covering it.

The floodlights used were 80 Watt, 240 Volt bulbs. Six globes of each colour were placed on the ceiling and four were placed on the sides. Two intensities of each coloured light were 
used, for the high intensity 10 globes of each coloured light were lit and for the low intensity 5 globes of each coloured light were lit. The lighting conditions were recorded using LUX and FC light meter as described in Table 3.1.

Table 3.1: Intensities of coloured lights

\begin{tabular}{|c|c|}
\hline Color of Lights & Intensity of light in Lux \\
\hline White & 207 \\
\hline Blue low & 28 \\
\hline Blue high & 48 \\
\hline Green low & 90 \\
\hline Green high & 169 \\
\hline Red low & 92 \\
\hline Red high & 157 \\
\hline Natural & $20-105$ \\
\hline
\end{tabular}

The duration of the complete experiment was approximately 110 minutes; participants were exposed to each coloured light for 10 minutes. The participants HR and SC were recorded in the last 2 minutes under each colour and intensity light, so they were given 8 minutes to adapt to the conditions before the recording. It was observed in preliminary experiments that 8 minutes were sufficient to ensure that participants adapted to the light and colour. A 3-minute break was given to the participants after being exposed to each lighting condition; during this time they were allowed to move and were asked to complete a paper and pencil version of SAM.

In order to ensure that there is clear recording of the physiological signals, the surface of the skin was prepared for the recoding. The skin of the wrist and leg was prepared for recording of $\mathrm{HR}$ using wipes. Three disposable $\mathrm{Ag} / \mathrm{AgCl}$ electrodes were firmly stuck for the duration of the complete experiment, two electrodes on each of the wrists and the ground electrode on the right ankle. The electrodes were attached as shown in Figure 3.1. The electrodes were packaged with wet gel by the manufacturer.

The fingers were prepared for recording SC using wipes. Two electrodes were wrapped around two fingers in the right hand for the duration of the complete experiment as shown in Figure 3.2. The participants' HR and SC were recorded in the last 2 minutes under each coloured light. 


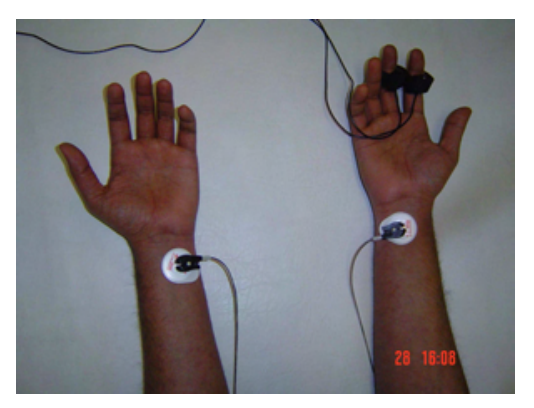

Figure 3.1: Electrode Placement for ECG Recordings

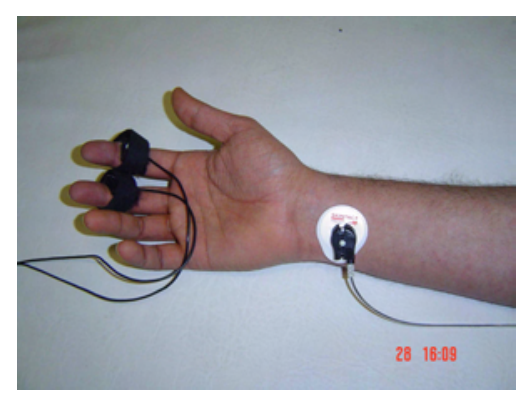

Figure 3.2: Electrode Placement for SC Recordings

\subsection{Equipments and signal processing}

$E C G$ and SC signals were recorded using AMLAB Biosignal recording equipment. SC was measured using Autogenics Single-Channel SCR Monitor that was connected to AMLAB. The sampling rate was set at 200 samples/second. The data were saved as text files and were further processed and analysed using MATLAB software.

To analyse the ECG signal first the RR interval was calculated using MATLAB software. The RR intervals were then exported to the Software Package Advanced HRV Analysis [46] where HR was calculated. The SC data was analysed using MATLAB software. The statistical analysis was performed using MINITAB software.

\subsection{Statistical analysis}

The statistical analysis was performed on the HR, SC and SAM results to determine if there was a change in the measures recorded due to change in the colour and intensity of lights. The statistical analysis was performed using the statistical software MINITAB.

One-way ANOVA was then performed on the data to determine the significance of the 
changes detected, $p$-value was calculated from ANOVA tests. The p-value is the probability of finding a difference between two group means. If the $p$-value is less than 0.05 , then the difference is significant and not caused by chance.

Two-way ANOVA with critical value or 0.05 and confidence level of $95 \%$ was also performed on HR, SC, arousal and valence values to determine the significance of the changes detected. The two factors considered were "colour" and "person". When "colour" was taken as a factor, changes in HR, SC and subjective tests of all participants were considered under each colour light. When "person" was taken as a factor, changes of HR, SC and subjective tests were considered for each person separately. In addition bar charts and graphs were used to assess the nature of the statistical data. 
CHAPTER 4

\section{Results and Observation}

he aim of this research is to study the effect of different colour and intensity lights on
the users of a constructed environment. Experiments were conducted towards this aim as explained in the Methodology chapter. This chapter presents the results of the experiments and tabulates all the data. A sample recording, arbitrarily chosen, is presented in this chapter while all recordings are shown in the Appendix.

\subsection{ECG results and analysis}

This section explains how HR was extracted from the ECG recording. The ECG signals were recorded for 2 minutes under each colour and intensity light for all subjects using AMLAB biosignal recording equipment. The data was saved as text files. These files were exported to MATLAB software for analysis. The raw ECG signal for participant 1 under White light is shown as an example in Figure 4.1.

$R$ peaks (RR interval) were calculated for each recording using MATLAB. These figures were then exported to the Advanced HRV Analysis software [HRV]. Using this software the mean RR, standard deviation (std) of RR, mean HR and std HR for each of the recordings were calculated. A sample of the results calculated using the Advanced HRV Analysis software for participant 10 is shown in Table 4.1 .

The mean HR for all participants has been tabulated. Using the Microsoft Excel software 


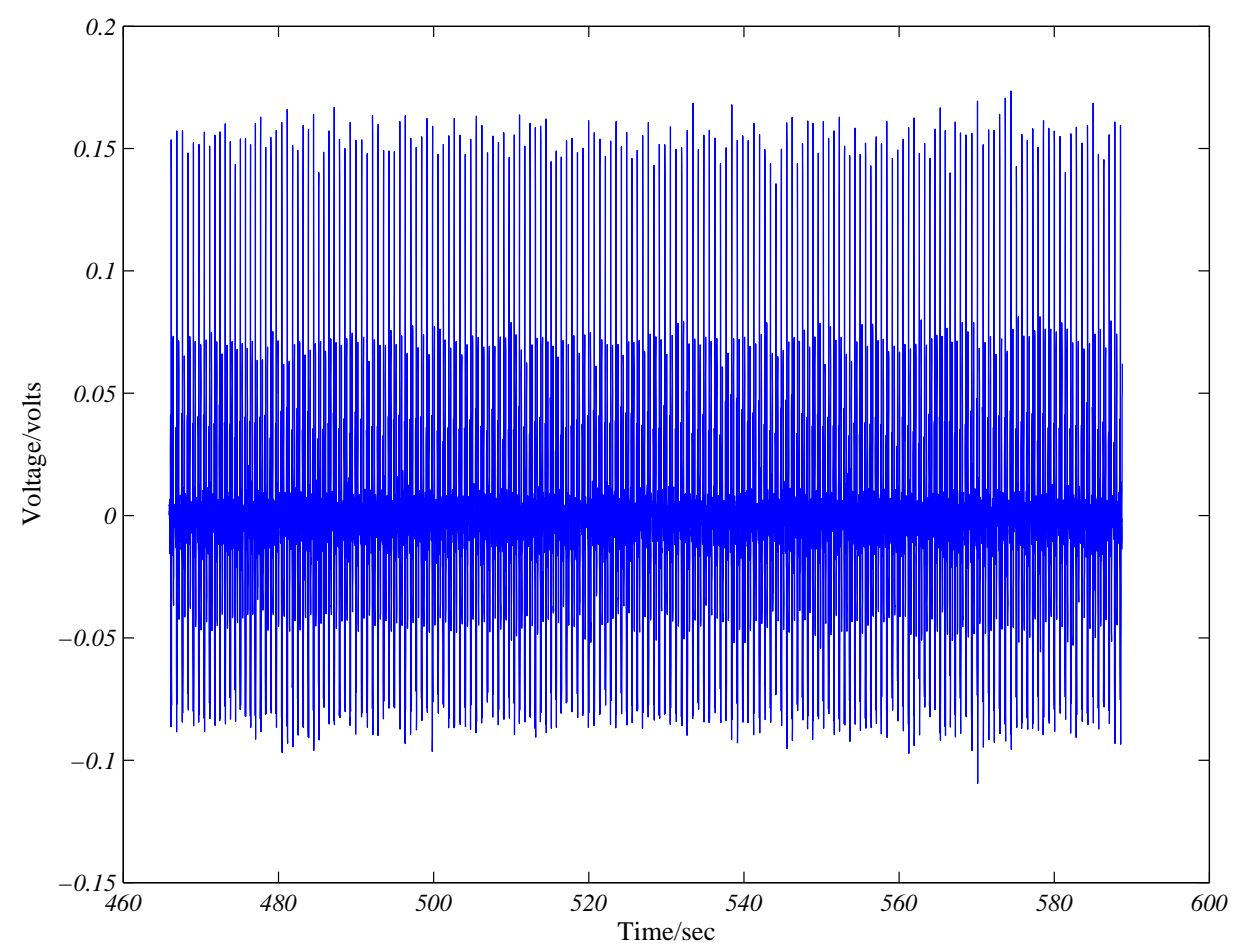

Figure 4.1: ECG signal for participant 1 under White light

Table 4.1: RR \& HR values for participant 10

\begin{tabular}{|c|c|c|c|c|c|c|c|c|c|}
\hline Parameters & Units & White & Blue-high & Blue-low & Green-high & Green-low & Red-high & Red-low & Natural \\
\hline Mean RR & sec & 0.73 & 0.70 & 0.72 & 0.70 & 0.72 & 0.75 & 0.68 & 0.65 \\
\hline std RR & sec & 0.197 & 0.175 & 0.148 & 0.165 & 0.158 & 0.153 & 0.185 & 0.125 \\
\hline Mean HR & per/min & 89.80 & 91.02 & 86.97 & 90.26 & 87.12 & 84.03 & 93.53 & 96.99 \\
\hline std HR & per/min & 26.09 & 23.14 & 18.11 & 21.96 & 17.12 & 18.59 & 21.49 & 19.81 \\
\hline
\end{tabular}

package, spread sheet calculation was used to calculate the mean and standard deviation (std) of HR for each participant. The mean HR for all participants under each colour light was also calculated. All these results are tabulated and are shown in Table 4.2.

It is observed from the results that there is variation in HR under different colour and intensity lights. For better comparison of the change seen in Table 4.2, the HR under white light was taken as a base level for each subject and the mean HR reading under all other colour lights were compared to it. Subtracting the mean HR under white light from the mean HR under each colour light provided these figures; the results are tabulated and shown in Table 4.3. 
Table 4.2: Mean HR (beats/min)

\begin{tabular}{|c|c|c|c|c|c|c|c|c|c|c|}
\hline Subject & White & Blue-low & Blue-high & Green-low & Green-high & Red-low & Red-high & Natural & Mean & SD \\
\hline 1 & 80.33 & 92.21 & 85.86 & 85.42 & 87.53 & 87.82 & 82.14 & 80.40 & 85.21 & 4.11 \\
\hline 2 & 89.59 & 77.09 & 82.24 & 83.67 & 87.12 & 81.24 & 79.54 & 82.27 & 82.85 & 4.00 \\
\hline 3 & 78.17 & 79.16 & 83.00 & 80.63 & 66.49 & 82.94 & 85.96 & 69.48 & 78.23 & 6.82 \\
\hline 4 & 73.68 & 78.80 & 82.78 & 84.11 & 78.50 & 81.61 & 75.62 & 78.74 & 79.23 & 3.52 \\
\hline 5 & 93.04 & 97.16 & 94.86 & 90.43 & 93.26 & 92.17 & 89.16 & 88.88 & 92.37 & 2.85 \\
\hline 6 & 83.25 & 88.62 & 93.01 & 82.09 & 84.27 & 81.29 & 89.90 & 77.73 & 85.02 & 5.07 \\
\hline 7 & 79.09 & 78.88 & 79.37 & 77.40 & 82.79 & 76.46 & 87.60 & 75.29 & 79.61 & 3.93 \\
\hline 8 & 71.52 & 81.02 & 78.03 & 89.14 & 72.08 & 85.82 & 78.52 & 80.02 & 79.52 & 6.06 \\
\hline 9 & 86.28 & 78.25 & 82.64 & 69.30 & 74.93 & 80.29 & 77.34 & 72.84 & 77.73 & 5.44 \\
\hline 10 & 89.80 & 86.97 & 91.02 & 87.12 & 90.26 & 93.53 & 84.03 & 96.99 & 89.97 & 4.06 \\
\hline 11 & 85.93 & 83.28 & 75.56 & 77.03 & 91.25 & 89.29 & 86.71 & 78.26 & 83.41 & 5.88 \\
\hline 12 & 90.82 & 90.27 & 93.47 & 93.94 & 89.78 & 94.36 & 82.53 & 87.63 & 90.35 & 3.92 \\
\hline 13 & 76.02 & 87.24 & 80.69 & 73.87 & 78.22 & 72.54 & 76.71 & 74.26 & 77.44 & 4.73 \\
\hline 14 & 89.43 & 86.19 & 84.24 & 84.01 & 87.00 & 82.97 & 78.95 & 83.73 & 84.57 & 3.10 \\
\hline 15 & 80.83 & 75.21 & 87.93 & 81.83 & 85.98 & 85.91 & 90.29 & 82.42 & 83.80 & 4.73 \\
\hline Mean & 83.19 & 84.02 & 84.98 & 82.67 & 83.30 & 84.55 & 83 & 80.60 & - & - \\
\hline SD & 6.71 & 6.38 & 5.91 & 6.45 & 7.72 & 6.18 & 5.06 & 6.93 & - & - \\
\hline
\end{tabular}

Table 4.3: Difference in mean HR

\begin{tabular}{|c|c|c|c|c|c|c|c|c|c|c|}
\hline Subject & White & Blue-low & Blue-high & Green-low & Green-high & Red-low & Red-high & Natural & Mean & SD \\
\hline 1 & 0 & 11.88 & 5.53 & 5.09 & 7.2 & 7.49 & 1.81 & 0.07 & 4.88 & 4.11 \\
\hline 2 & 0 & -12.5 & -7.35 & -5.92 & -2.47 & -8.35 & -10.05 & -7.32 & -6.75 & 4.00 \\
\hline 3 & 0 & 0.99 & 4.83 & 2.46 & -11.68 & 4.77 & 7.79 & -8.69 & 0.06 & 6.82 \\
\hline 4 & 0 & 5.12 & 9.1 & 10.43 & 4.82 & 7.93 & 1.94 & 5.06 & 5.55 & 3.52 \\
\hline 5 & 0 & 4.12 & 1.82 & -2.61 & 0.22 & -0.87 & -3.88 & -4.16 & -0.67 & 2.85 \\
\hline 6 & 0 & 5.37 & 9.76 & -1.16 & 1.02 & -1.96 & 6.65 & -5.52 & 1.77 & 5.07 \\
\hline 7 & 0 & -0.21 & 0.28 & -1.69 & 3.7 & -2.63 & 8.51 & -3.8 & 0.52 & 3.93 \\
\hline 8 & 0 & 9.5 & 6.51 & 17.62 & 0.56 & 14.3 & 7 & 8.5 & 8.00 & 6.06 \\
\hline 9 & 0 & -8.03 & -3.64 & -16.98 & -11.35 & -5.99 & -8.94 & -13.44 & -8.55 & 5.44 \\
\hline 10 & 0 & -2.83 & 1.22 & -2.68 & 0.46 & 3.73 & -5.77 & 7.19 & 0.17 & 4.06 \\
\hline 11 & 0 & -2.65 & -10.37 & -8.9 & 5.32 & 3.36 & 0.78 & -7.67 & -2.52 & 5.88 \\
\hline 12 & 0 & -0.55 & 2.65 & 3.12 & -1.04 & 3.54 & -8.29 & -3.19 & -0.47 & 3.92 \\
\hline 13 & 0 & 11.22 & 4.67 & -2.15 & 2.2 & -3.48 & 0.69 & -1.76 & 1.42 & 4.73 \\
\hline 14 & 0 & -3.24 & -5.19 & -5.42 & -2.43 & -6.46 & -10.48 & -5.7 & -4.87 & 3.10 \\
\hline 15 & 0 & -5.62 & 7.1 & 1 & 5.15 & 5.08 & 9.46 & 1.59 & 2.97 & 4.73 \\
\hline Mean & 0 & 0.84 & 1.79 & -0.52 & 0.11 & 1.36 & -0.19 & -2.59 & - & - \\
\hline SD & 0 & 7.06 & 6.05 & 8.07 & 5.53 & 6.28 & 7.23 & 6.14 & - & - \\
\hline
\end{tabular}


For more comparison the results were also normalised for each subject by taking the mean HR under white light as a reference. Dividing the mean HR under each colour light by the mean HR under white light gave these figures. The results are tabulated and shown in Table 4.4.

Table 4.4: Normalised values of mean HR (White light as reference)

\begin{tabular}{|c|c|c|c|c|c|c|c|c|c|c|}
\hline Subject & White & Blue-low & Blue-high & Green-low & Green-high & Red-low & Red-high & Natural & Mean & SD \\
\hline 1 & 1 & 1.15 & 1.07 & 1.06 & 1.09 & 1.09 & 1.02 & 1.00 & 1.06 & 0.05 \\
\hline 2 & 1 & 0.86 & 0.92 & 0.93 & 0.97 & 0.91 & 0.89 & 0.92 & 0.92 & 0.04 \\
\hline 3 & 1 & 1.01 & 1.06 & 1.03 & 0.85 & 1.06 & 1.1 & 0.89 & 1.00 & 0.09 \\
\hline 4 & 1 & 1.07 & 1.12 & 1.14 & 1.07 & 1.11 & 1.03 & 1.07 & 1.08 & 0.05 \\
\hline 5 & 1 & 1.04 & 1.02 & 0.97 & 1.00 & 0.99 & 0.96 & 0.96 & 0.99 & 0.03 \\
\hline 6 & 1 & 1.07 & 1.12 & 0.99 & 1.01 & 0.98 & 1.08 & 0.93 & 1.02 & 0.06 \\
\hline 7 & 1 & 1.00 & 1.00 & 0.98 & 1.05 & 0.97 & 1.11 & 0.95 & 1.01 & 0.05 \\
\hline 8 & 1 & 1.13 & 1.09 & 1.25 & 1.01 & 1.2 & 1.10 & 1.12 & 1.11 & 0.08 \\
\hline 9 & 1 & 0.91 & 0.96 & 0.80 & 0.87 & 0.93 & 0.90 & 0.84 & 0.90 & 0.06 \\
\hline 10 & 1 & 0.97 & 1.01 & 0.97 & 1.01 & 1.04 & 0.94 & 1.08 & 1.00 & 0.05 \\
\hline 11 & 1 & 0.97 & 0.88 & 0.90 & 1.06 & 1.04 & 1.01 & 0.91 & 0.97 & 0.07 \\
\hline 12 & 1 & 0.99 & 1.03 & 1.03 & 0.99 & 1.04 & 0.91 & 0.97 & 0.99 & 0.04 \\
\hline 13 & 1 & 1.15 & 1.06 & 0.97 & 1.03 & 0.95 & 1.01 & 0.98 & 1.02 & 0.06 \\
\hline 14 & 1 & 0.96 & 0.94 & 0.94 & 0.97 & 0.93 & 0.88 & 0.94 & 0.95 & 0.03 \\
\hline 15 & 1 & 0.93 & 1.09 & 1.01 & 1.06 & 1.06 & 1.12 & 1.02 & 1.04 & 0.06 \\
\hline Mean & 1 & 1.01 & 1.03 & 1.00 & 1.00 & 1.02 & 1.00 & 0.97 & - & - \\
\hline SD & 0 & 0.09 & 0.07 & 0.10 & 0.07 & 0.08 & 0.09 & 0.08 & - & - \\
\hline
\end{tabular}

Using Excel software, the difference in mean HR obtained from table 3 were put into bar charts. An example for participant 5 is shown in Figure 4.2.

It is observed from Table 4.3, Table 4.4 and Figure 4.2 that there is variation in HR under different colour and intensity lights. It is also noted that some colours and intensities caused greater changes in HR than others. Red high light caused the largest increase in HR for $20 \%$ of the subjects and the largest decrease in HR for $20 \%$ of the subjects. Blue low light caused the largest increase in HR for $20 \%$ of the subjects and the largest decrease in HR for $13 \%$ of the subjects. Natural light caused the largest decrease in HR for $20 \%$ of the subjects. It is also observed that the direction of change in HR (increase or decrease) is subject dependent, where the same colour and intensity light can cause an increase in HR of some subjects and a decrease in HR for others. Also the inter subject variation in HR is very large. That can be seen clearly from the last rows in Table 4.3 \& Table 4.4 where the variation in HR between different 


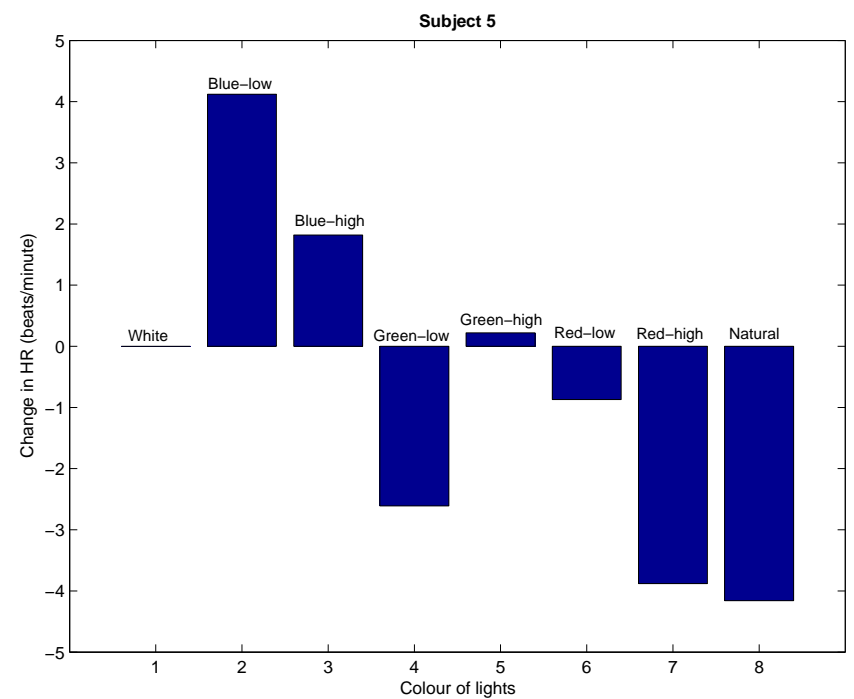

Figure 4.2: Difference in mean HR for subject 5

colour and intensity lights is relatively small compared to the inter subject variation.

\subsection{SC results and analysis}

SC of participants was recorded for 2 minutes under each colour and intensity light using AMLAB biosignal recording equipment. The data was saved as text files, these files were then exported to MATLAB software for analysis. Using MATLAB, the SC recordings for each participant were put into graphs. Figure 4.3 shows SC graph for subject 10.

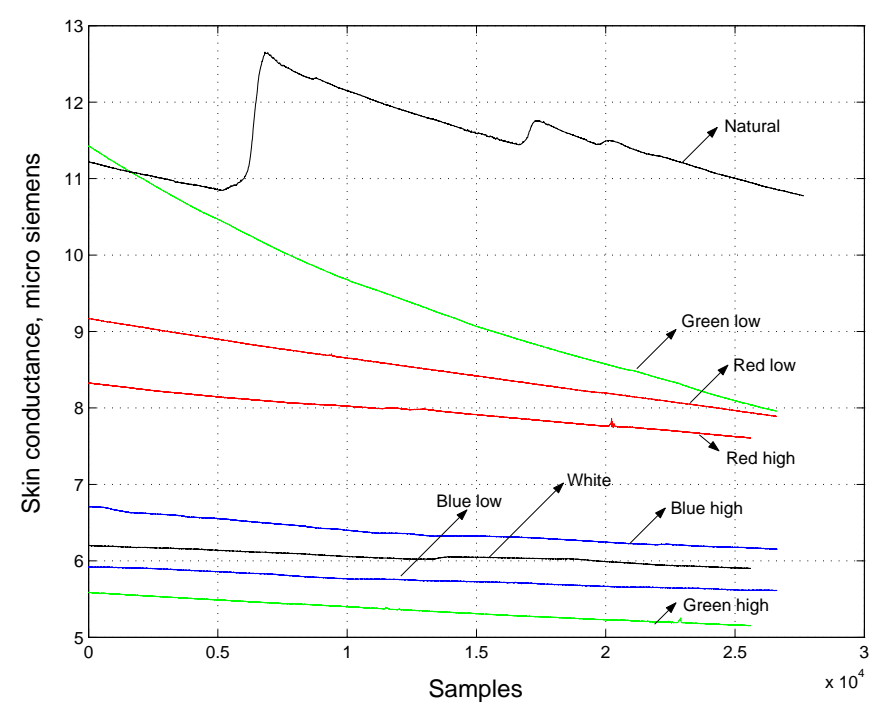

Figure 4.3: SC signal for participant 10 
Using the same software, the values of SC were averaged over the two minutes for each recording. These values were tabulated. The mean and standard deviation (std) of SC for each participant were then calculated using Excel. The mean and std of SC for all participants under each colour light were tabulated and the results are shown in Table 4.5.

It is observed from Table 4.5 that there is variation in SC under different colour and intensity lights. The SC under white light was then taken as a base level for each subject and SC readings under all other colour lights were compared to it. Subtracting the mean SC under white light from each coloured light provided these figures; the results are tabulated and shown in Table 4.6.

Table 4.5: Mean SC values

\begin{tabular}{|c|c|c|c|c|c|c|c|c|c|c|}
\hline Subject & White & Blue-low & Blue-high & Green-low & Green-high & Red-low & Red-high & Natural & Mean & SD \\
\hline 1 & 20.38 & 13.65 & 16.18 & 19.95 & 13.59 & 19.61 & 20.38 & 21.34 & 18.14 & 3.17 \\
\hline 2 & 7.9 & 9.31 & 7.64 & 6.8 & 6.22 & 6.13 & 5.22 & 6.09 & 6.91 & 1.30 \\
\hline 3 & 16.5 & 27.12 & 26.54 & 99.91 & 99.92 & 64.62 & 17.36 & 41.39 & 49.17 & 34.90 \\
\hline 4 & 60.7 & 61.22 & 59.12 & 56.72 & 54.61 & 53.1 & 51.89 & 51.05 & 56.05 & 3.99 \\
\hline 5 & 9.14 & 12.71 & 11.09 & 10.49 & 11.37 & 8.58 & 10.07 & 7.45 & 10.11 & 1.68 \\
\hline 6 & 13.99 & 15.55 & 23.77 & 31.24 & 16.85 & 18.5 & 21.81 & 25.25 & 20.87 & 5.77 \\
\hline 7 & 27.13 & 50.31 & 49.95 & 39.22 & 43.57 & 55.28 & 53.89 & 69.56 & 48.61 & 12.48 \\
\hline 8 & 55.74 & 25.6 & 65.09 & 47.32 & 90.25 & 99.92 & 99.92 & 99.92 & 72.97 & 28.63 \\
\hline 9 & 30.44 & 15.06 & 13.88 & 43.02 & 38.7 & 33.98 & 45.66 & 37.46 & 32.28 & 11.97 \\
\hline 10 & 6.05 & 5.75 & 6.38 & 9.41 & 5.36 & 8.51 & 7.96 & 11.47 & 7.61 & 2.12 \\
\hline 11 & 40.04 & 55.35 & 50.35 & 51.93 & 57.88 & 59.87 & 63.02 & 47.25 & 53.21 & 7.42 \\
\hline 12 & 25.04 & 28.55 & 30.77 & 35.59 & 37.57 & 35.05 & 42.08 & 35.96 & 33.83 & 5.42 \\
\hline 13 & 38.2 & 74.78 & 64.01 & 23.62 & 28.94 & 29.13 & 24.36 & 40.91 & 40.49 & 19.05 \\
\hline 14 & 7.46 & 7.07 & 6.84 & 6.63 & 7.61 & 7.78 & 6.72 & 5.68 & 6.97 & 0.67 \\
\hline 15 & 8.1 & 9.6 & 9.42 & 10.07 & 11.57 & 11.33 & 11.14 & 10.52 & 10.22 & 1.17 \\
\hline Mean & 24.45 & 27.44 & 29.40 & 32.79 & 34.93 & 34.09 & 32.10 & 34.09 & - & - \\
\hline SD & 17.65 & 22.24 & 22.23 & 25.33 & 30.07 & 27.41 & 26.86 & 26.42 & - & - \\
\hline
\end{tabular}

The results were also normalised for each subject by taking the mean SC under white light as a reference, the results are tabulated and shown in Table 4.7.

Using Excel, the difference in mean SC obtained from Table 4.6 was then put into bar charts for comparison. An example for subject 10 is shown in Figure 4.4. 
Table 4.6: Difference in mean SC

\begin{tabular}{|c|c|c|c|c|c|c|c|c|c|c|}
\hline Subject & White & Blue-low & Blue-high & Green-low & Green-high & Red-low & Red-high & Natural & Mean & SD \\
\hline 1 & 0 & -6.73 & -4.2 & -0.43 & -6.78 & -0.77 & 0.01 & 0.97 & -2.56 & 3.17 \\
\hline 2 & 0 & 1.4 & -0.26 & -1.11 & -1.69 & -1.77 & -2.68 & -1.81 & -1.13 & 1.30 \\
\hline 3 & 0 & 10.62 & 10.04 & 83.42 & 83.42 & 48.13 & 0.86 & 24.89 & 37.34 & 34.90 \\
\hline 4 & 0 & 0.52 & -1.58 & -3.98 & -6.09 & -7.6 & -8.81 & -9.65 & -5.31 & 3.99 \\
\hline 5 & 0 & 3.57 & 1.95 & 1.35 & 2.22 & -0.57 & 0.93 & -1.69 & 1.11 & 1.68 \\
\hline 6 & 0 & 1.56 & 9.78 & 17.25 & 2.86 & 4.51 & 7.83 & 11.26 & 7.86 & 5.77 \\
\hline 7 & 0 & 23.18 & 22.82 & 12.09 & 16.44 & 28.15 & 26.76 & 42.43 & 24.55 & 12.48 \\
\hline 8 & 0 & -30.14 & 9.36 & -8.41 & 34.51 & 44.18 & 44.18 & 44.18 & 19.69 & 28.63 \\
\hline 9 & 0 & -15.38 & -16.56 & 12.58 & 8.25 & 3.54 & 15.21 & 7.02 & 2.09 & 11.97 \\
\hline 10 & 0 & -0.3 & 0.32 & 3.36 & -0.7 & 2.45 & 1.9 & 5.42 & 1.78 & 2.12 \\
\hline 11 & 0 & 15.31 & 10.3 & 11.89 & 17.84 & 19.83 & 22.97 & 7.21 & 15.05 & 7.42 \\
\hline 12 & 0 & 3.51 & 5.73 & 10.55 & 12.53 & 10.01 & 17.05 & 10.92 & 10.04 & 5.42 \\
\hline 13 & 0 & 36.58 & 25.81 & -14.58 & -9.27 & -9.07 & -13.84 & 2.7 & 2.62 & 19.06 \\
\hline 14 & 0 & -0.39 & -0.62 & -0.83 & 0.16 & 0.32 & -0.74 & -1.78 & -0.55 & 0.67 \\
\hline 15 & 0 & 1.49 & 1.32 & 1.97 & 3.47 & 3.23 & 3.04 & 2.42 & 2.42 & 1.17 \\
\hline Mean & - & 2.99 & 4.95 & 8.34 & 10.48 & 9.64 & 7.64 & 9.63 & - & - \\
\hline SD & - & 15.38 & 10.50 & 22.50 & 23.17 & 17.64 & 15.10 & 15.75 & - & - \\
\hline
\end{tabular}

Table 4.7: Normalised values of mean SC (white light as reference)

\begin{tabular}{|c|c|c|c|c|c|c|c|c|c|c|}
\hline Subject & White & Blue-low & Blue-high & Green-low & Green-high & Red-low & Red-high & Natural & Mean & SD \\
\hline 1 & 1 & 0.67 & 0.79 & 0.98 & 0.67 & 0.96 & 1.00 & 1.05 & 0.87 & 0.16 \\
\hline 2 & 1 & 1.18 & 0.97 & 0.86 & 0.79 & 0.78 & 0.66 & 0.77 & 0.86 & 0.17 \\
\hline 3 & 1 & 1.64 & 1.61 & 6.06 & 6.06 & 3.92 & 1.05 & 2.51 & 3.26 & 2.12 \\
\hline 4 & 1 & 1.01 & 0.97 & 0.93 & 0.90 & 0.87 & 0.85 & 0.84 & 0.91 & 0.06 \\
\hline 5 & 1 & 1.39 & 1.21 & 1.15 & 1.24 & 0.94 & 1.10 & 0.82 & 1.12 & 0.19 \\
\hline 6 & 1 & 1.11 & 1.70 & 2.23 & 1.20 & 1.32 & 1.56 & 1.80 & 1.56 & 0.39 \\
\hline 7 & 1 & 1.85 & 1.84 & 1.45 & 1.61 & 2.04 & 1.99 & 2.56 & 1.91 & 0.36 \\
\hline 8 & 1 & 0.46 & 1.17 & 0.85 & 1.62 & 1.79 & 1.79 & 1.79 & 1.35 & 0.54 \\
\hline 9 & 1 & 0.49 & 0.46 & 1.41 & 1.27 & 1.12 & 1.50 & 1.23 & 1.07 & 0.42 \\
\hline 10 & 1 & 0.95 & 1.05 & 1.56 & 0.89 & 1.41 & 1.32 & 1.90 & 1.29 & 0.36 \\
\hline 11 & 1 & 1.38 & 1.26 & 1.30 & 1.45 & 1.50 & 1.57 & 1.18 & 1.38 & 0.14 \\
\hline 12 & 1 & 1.14 & 1.23 & 1.42 & 1.50 & 1.40 & 1.68 & 1.44 & 1.40 & 0.18 \\
\hline 13 & 1 & 1.96 & 1.68 & 0.62 & 0.76 & 0.76 & 0.64 & 1.07 & 1.07 & 0.54 \\
\hline 14 & 1 & 0.95 & 0.92 & 0.89 & 1.02 & 1.04 & 0.90 & 0.76 & 0.93 & 0.09 \\
\hline 15 & 1 & 1.19 & 1.16 & 1.24 & 1.43 & 1.40 & 1.38 & 1.30 & 1.30 & 0.11 \\
\hline Mean & - & 1.16 & 1.20 & 1.53 & 1.49 & 1.42 & 1.27 & 1.40 & - & - \\
\hline SD & - & 0.44 & 0.38 & 1.31 & 1.30 & 0.78 & 0.42 & 0.59 & - & - \\
\hline
\end{tabular}




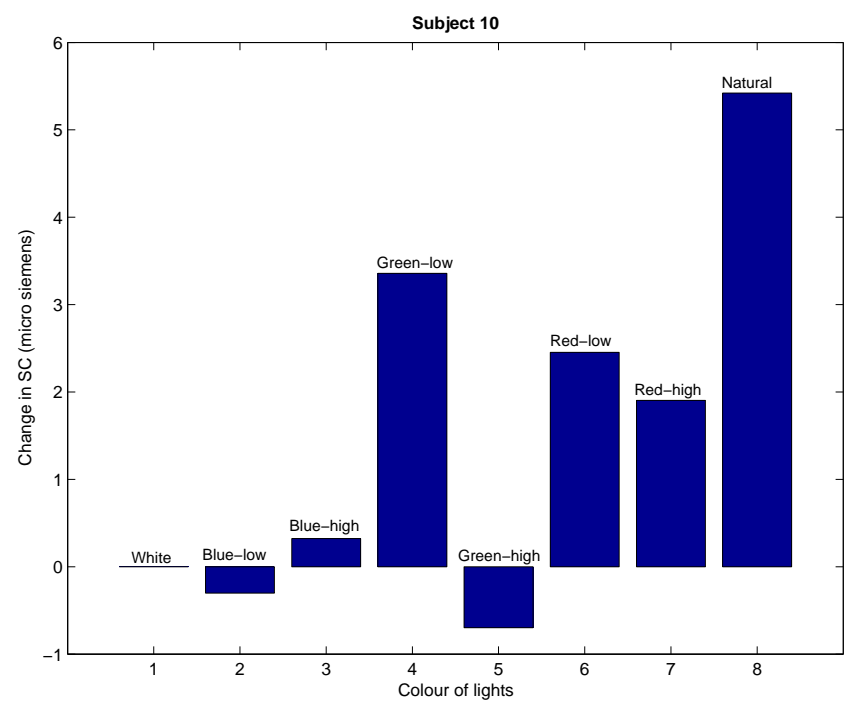

Figure 4.4: Change in mean SC for participant 10

It is observed from Table 4.6, Table 4.7 and the barcharts that $60-73 \%$ of the non-white conditions caused higher SC than white conditions. It is also noted that some colours caused greater change in SC than other colours. Natural light caused the largest increase in SC for $20 \%$ of the subjects and the largest decrease in SC for $20 \%$ of subjects. Green high light caused the largest increase in SC for $13 \%$ of the subjects and the largest decrease in SC for $13 \%$ of the subjects. Red high light caused the largest increase in SC for $20 \%$ of the subjects and blue low light caused the largest decrease in SC for $20 \%$ of the subjects. It has also been observed that the direction of change in SC (increase or decrease) is subject dependent, where the same colour and intensity light can cause an increase in SC for some subjects and a decrease in SC for others. However it is also noted from the results that the inter subject variation is large. That can be seen clearly from the last rows in Table 4.6 \& Table 4.7 where the variation in SC between different colour and intensity lights is relatively small compared to the inter subject variation.

\subsection{SAM}

Participants indicated their reactions to different colour and intensity lights by providing valence and arousal rating in SAM. SAM is a nine-point rating ranging from -4 to 4 for each dimension. For the arousal dimension, SAM ranges from an excited, wide-eyed figure (rated 4) to a relaxed sleepy figure (rated -4$)$. For the valence dimension, SAM ranges from a smiling happy figure 
(rated 4) to a frowning unhappy figure (rated -4).

\subsubsection{Arousal}

Participants were asked to give their arousal rating after being exposed to each colour and intensity light. The figure ratings were then transferred to numbers (-4 to 4$)$. Using Excel, the mean and std arousal of all participants under each colour light were calculated. The results are tabulated and shown in Table 4.8.

The results were then put into bar charts for comparison, an example for participant 3 is shown as an example in Figure 4.5.

Table 4.8: Values of arousal rating

\begin{tabular}{|c|c|c|c|c|c|c|c|c|}
\hline Subject & White & Blue-low & Blue-high & Green-low & Green-high & Red-low & Red-high & Natural \\
\hline 1 & 0 & 1 & -2 & -4 & 0 & 2 & 3 & 3 \\
\hline 2 & 0 & -1 & -2 & 2 & 4 & -2 & -2 & -4 \\
\hline 3 & -3 & -2 & -1 & 1 & 2 & 2 & 3 & 0 \\
\hline 4 & 0 & 2 & -1 & 0 & 0 & 2 & -2 & -4 \\
\hline 5 & -4 & -2 & -2 & 0 & 1 & 1 & 4 & 4 \\
\hline 6 & 0 & -1 & -2 & -2 & 1 & -1 & 0 & 2 \\
\hline 7 & 0 & -2 & -4 & -1 & -2 & -1 & 0 & 3 \\
\hline 8 & 0 & 1 & 2 & 1 & 2 & 0 & 1 & 2 \\
\hline 9 & 0 & -3 & -4 & 0 & 2 & 2 & 2 & 2 \\
\hline 10 & 4 & 3 & 4 & 4 & 3 & 4 & 4 & 4 \\
\hline 11 & 0 & 0 & -2 & 0 & -2 & 2 & 3 & -3 \\
\hline 12 & 0 & -2 & 0 & 2 & 0 & 2 & 2 & 0 \\
\hline 13 & 2 & 4 & 2 & 3 & 0 & 0 & 1 & -2 \\
\hline 14 & -2 & -4 & -4 & -2 & 0 & 0 & 0 & -1 \\
\hline 15 & 0 & -3 & -4 & -3 & -2 & -1 & 0 & -3 \\
\hline Mean & -0.2 & -0.6 & -1.33 & 0.07 & 0.6 & 0.8 & 1.27 & 0.2 \\
\hline SD & 1.86 & 2.35 & 2.44 & 2.22 & 1.80 & 1.66 & 1.94 & 2.88 \\
\hline
\end{tabular}

It is observed from Table 4.8 and the barcharts that there is a change in arousal rating under different colour and intensity lights. These changes were also subject dependent, where the same colour and intensity light can cause different arousal effects on different people. 


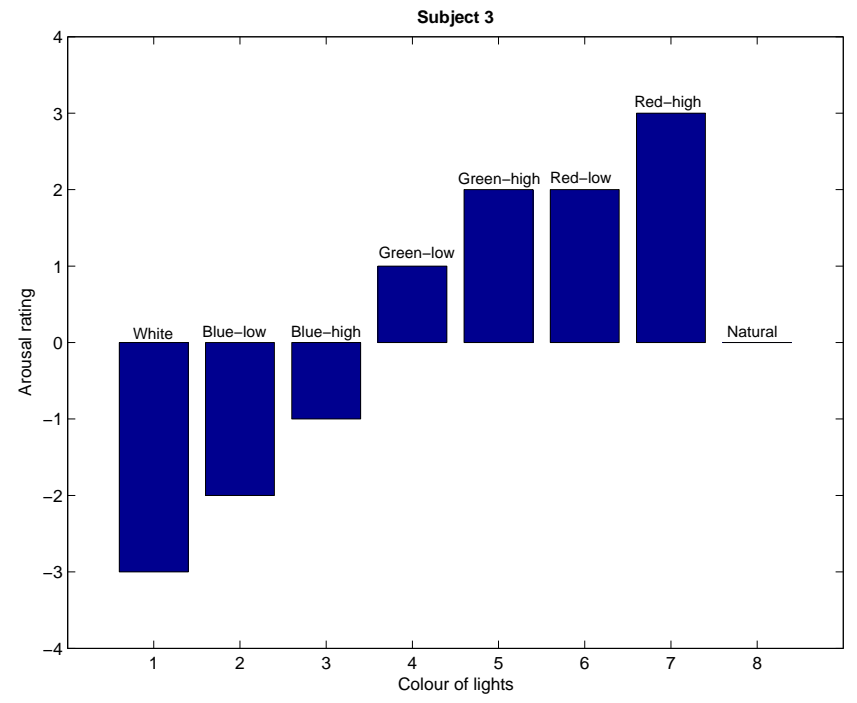

Figure 4.5: Change in arousal participant 3

Table 4.9: 3 -point scale of arousal ( $\mathrm{N}=$ neutral, $\mathrm{E}=$ excited, $\mathrm{R}=$ relaxed)

\begin{tabular}{|c|c|c|c|c|c|c|c|c|}
\hline Subject & White & Blue-low & Blue-high & Green-low & Green-high & Red-low & Red-high & Natural \\
\hline 1 & $\mathrm{~N}$ & $\mathrm{E}$ & $\mathrm{R}$ & $\mathrm{R}$ & $\mathrm{N}$ & $\mathrm{E}$ & $\mathrm{E}$ & $\mathrm{E}$ \\
\hline 2 & $\mathrm{~N}$ & $\mathrm{R}$ & $\mathrm{R}$ & $\mathrm{E}$ & $\mathrm{E}$ & $\mathrm{R}$ & $\mathrm{R}$ & $\mathrm{R}$ \\
\hline 3 & $\mathrm{R}$ & $\mathrm{R}$ & $\mathrm{R}$ & $\mathrm{E}$ & $\mathrm{E}$ & $\mathrm{E}$ & $\mathrm{E}$ & $\mathrm{N}$ \\
\hline 4 & $\mathrm{~N}$ & $\mathrm{E}$ & $\mathrm{R}$ & $\mathrm{N}$ & $\mathrm{N}$ & $\mathrm{E}$ & $\mathrm{R}$ & $\mathrm{R}$ \\
\hline 5 & $\mathrm{R}$ & $\mathrm{R}$ & $\mathrm{R}$ & $\mathrm{N}$ & $\mathrm{E}$ & $\mathrm{E}$ & $\mathrm{E}$ & $\mathrm{E}$ \\
\hline 6 & $\mathrm{~N}$ & $\mathrm{R}$ & $\mathrm{R}$ & $\mathrm{R}$ & $\mathrm{E}$ & $\mathrm{R}$ & $\mathrm{N}$ & $\mathrm{E}$ \\
\hline 7 & $\mathrm{~N}$ & $\mathrm{R}$ & $\mathrm{R}$ & $\mathrm{R}$ & $\mathrm{R}$ & $\mathrm{R}$ & $\mathrm{N}$ & $\mathrm{E}$ \\
\hline 8 & $\mathrm{~N}$ & $\mathrm{E}$ & $\mathrm{E}$ & $\mathrm{E}$ & $\mathrm{E}$ & $\mathrm{N}$ & $\mathrm{E}$ & $\mathrm{E}$ \\
\hline 9 & $\mathrm{~N}$ & $\mathrm{R}$ & $\mathrm{R}$ & $\mathrm{N}$ & $\mathrm{E}$ & $\mathrm{E}$ & $\mathrm{E}$ & $\mathrm{E}$ \\
\hline 10 & $\mathrm{E}$ & $\mathrm{E}$ & $\mathrm{E}$ & $\mathrm{E}$ & $\mathrm{E}$ & $\mathrm{E}$ & $\mathrm{E}$ & $\mathrm{E}$ \\
\hline 11 & $\mathrm{~N}$ & $\mathrm{~N}$ & $\mathrm{R}$ & $\mathrm{N}$ & $\mathrm{R}$ & $\mathrm{E}$ & $\mathrm{E}$ & $\mathrm{R}$ \\
\hline 12 & $\mathrm{~N}$ & $\mathrm{R}$ & $\mathrm{N}$ & $\mathrm{E}$ & $\mathrm{N}$ & $\mathrm{E}$ & $\mathrm{E}$ & $\mathrm{N}$ \\
\hline 13 & $\mathrm{E}$ & $\mathrm{E}$ & $\mathrm{E}$ & $\mathrm{E}$ & $\mathrm{N}$ & $\mathrm{E}$ & $\mathrm{E}$ & $\mathrm{R}$ \\
\hline 14 & $\mathrm{R}$ & $\mathrm{R}$ & $\mathrm{R}$ & $\mathrm{R}$ & $\mathrm{N}$ & $\mathrm{N}$ & $\mathrm{N}$ & $\mathrm{R}$ \\
\hline 15 & $\mathrm{~N}$ & $\mathrm{R}$ & $\mathrm{R}$ & $\mathrm{R}$ & $\mathrm{R}$ & $\mathrm{R}$ & $\mathrm{N}$ & $\mathrm{R}$ \\
\hline
\end{tabular}


For further analysis, the 9-point scale was modified to a 3-point scale. That was done by considering all positive values to be 1-point (excited) and considering all negative values to be 1-point (relaxed). Zero values were considered as neutral. The modified 3-point scale was done manually and is shown in Table 4.9.

Although the changes in arousal were subject dependent, some general trends could be drawn from Table 4.9. It was observed that the white light caused a neutral effect on $67 \%$ of participants, blue low light caused a relaxing effect on $60 \%$ of participants and blue high light caused a relaxing effect on $73 \%$ of participants. Red low light caused an exciting effect on $53 \%$ of participants and red high light caused an exciting effect on $60 \%$ of participants.

\subsubsection{Valence}

Participants were asked to give their valence rating after being exposed to each colour and intensity light. The figure ratings were then transferred to numbers (-4 to 4). Using Excel, the mean and std valence of all participants under each colour light were calculated. The results are tabulated and shown in Table 4.10.

Table 4.10: Values of valence rating

\begin{tabular}{|c|c|c|c|c|c|c|c|c|}
\hline Subject & White & Blue-low & Blue-high & Green-low & Green-high & Red-low & Red-high & Natural \\
\hline 1 & 0 & -3 & -2 & 2 & 4 & 3 & 2 & 4 \\
\hline 2 & 0 & 1 & 1 & 2 & 4 & 0 & 2 & 0 \\
\hline 3 & 0 & 2 & -1 & 2 & -1 & 0 & 2 & 2 \\
\hline 4 & 2 & 2 & 0 & 0 & 2 & 2 & 0 & 0 \\
\hline 5 & 2 & 2 & 2 & 2 & 2 & 2 & 4 & 4 \\
\hline 6 & -2 & -1 & 0 & -1 & 1 & 0 & -1 & 2 \\
\hline 7 & 0 & 0 & 0 & 1 & 1 & 0 & 2 & 3 \\
\hline 8 & 0 & 1 & 1 & 2 & 2 & 1 & 1 & 2 \\
\hline 9 & 0 & 0 & 0 & 0 & 2 & 2 & 2 & 2 \\
\hline 10 & 4 & 4 & 4 & 4 & 3 & 4 & 4 & 4 \\
\hline 11 & 1 & 0 & -1 & 2 & 2 & -2 & -4 & 4 \\
\hline 12 & 4 & 2 & 0 & 2 & 4 & -1 & -2 & 0 \\
\hline 13 & 3 & 4 & 2 & 2 & 2 & -2 & -2 & 0 \\
\hline 14 & 0 & 1 & 1 & 2 & 2 & 2 & 2 & 1 \\
\hline 15 & 0 & 1 & 1 & 2 & 3 & 1 & 2 & 3 \\
\hline Mean & 0.93 & 1.07 & 0.53 & 1.6 & 2.2 & 0.8 & 0.93 & 2.07 \\
\hline SD & 1.71 & 1.79 & 1.46 & 1.18 & 1.32 & 1.74 & 2.28 & 1.58 \\
\hline
\end{tabular}


The results were then put into bar charts for comparison, an example for participant 3 is shown as an example in Figure 4.6.

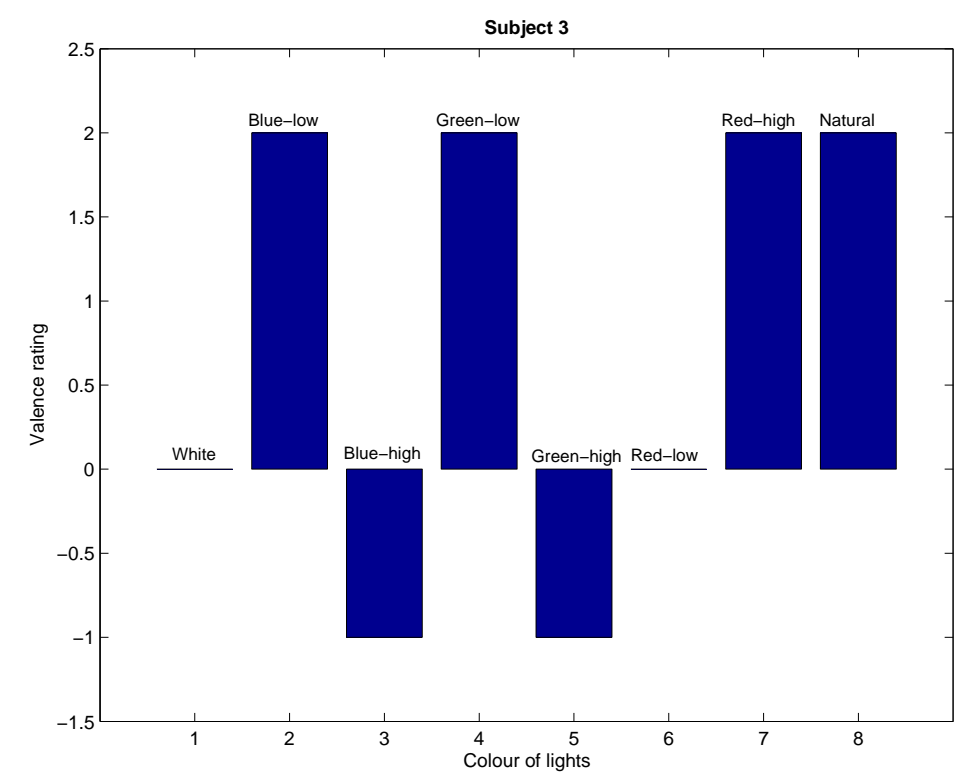

Figure 4.6: Change in valence participant 3

It is observed from Table 4.10 and the barcharts that there is a change in valence rating under different colour and intensity lights. These changes were also subject dependent, where the same colour and intensity light can cause different valence effects on different people.

For further analysis, the 9-point scale was modified to a 3-point scale. That was done by considering all positive values to be 1-point (happy) and considering all negative values to be 1-point (unhappy). Zero values were considered as neutral. The modified 3-point scale was done manually and is shown in Table 4.11.

It is observed from Table 4.11 that there are some colour and intensity lights that caused a very high percentage of pleasant happy effect on participants. Green high light caused a happy effect on $93 \%$ of participants; green low caused the same effect on $80 \%$ of participants and natural light caused that effect on $73 \%$ of participants. It was observed that all colours and intensities caused a happy or neutral effect on most participants. Some colour lights like green low, green high and white caused an unpleasant effect on only $7 \%$ of the participants. Blue low caused an unpleasant effect on $13 \%$ of participants, blue high and red low caused that effect for $20 \%$ of participants, while red high caused it for $26 \%$ of participants. Natural light did not cause an unpleasant effect for any participants. 
Table 4.11: 3 -point scale of valence ( $\mathrm{N}=$ neutral, $\mathrm{H}=$ happy, $\mathrm{UH}=$ unhappy).

\begin{tabular}{|c|c|c|c|c|c|c|c|c|}
\hline Subject & White & Blue-low & Blue-high & Green-low & Green-high & Red-low & Red-high & Natural \\
\hline 1 & $\mathrm{~N}$ & $\mathrm{UH}$ & $\mathrm{UH}$ & $\mathrm{H}$ & $\mathrm{H}$ & $\mathrm{H}$ & $\mathrm{H}$ & $\mathrm{H}$ \\
\hline 2 & $\mathrm{~N}$ & $\mathrm{H}$ & $\mathrm{H}$ & $\mathrm{H}$ & $\mathrm{H}$ & $\mathrm{N}$ & $\mathrm{H}$ & $\mathrm{N}$ \\
\hline 3 & $\mathrm{~N}$ & $\mathrm{H}$ & $\mathrm{UH}$ & $\mathrm{H}$ & $\mathrm{UH}$ & $\mathrm{N}$ & $\mathrm{H}$ & $\mathrm{H}$ \\
\hline 4 & $\mathrm{H}$ & $\mathrm{H}$ & $\mathrm{N}$ & $\mathrm{N}$ & $\mathrm{H}$ & $\mathrm{H}$ & $\mathrm{N}$ & $\mathrm{N}$ \\
\hline 5 & $\mathrm{H}$ & $\mathrm{H}$ & $\mathrm{H}$ & $\mathrm{H}$ & $\mathrm{H}$ & $\mathrm{H}$ & $\mathrm{H}$ & $\mathrm{H}$ \\
\hline 6 & $\mathrm{UH}$ & $\mathrm{UH}$ & $\mathrm{N}$ & $\mathrm{UH}$ & $\mathrm{H}$ & $\mathrm{N}$ & $\mathrm{UH}$ & $\mathrm{H}$ \\
\hline 7 & $\mathrm{~N}$ & $\mathrm{~N}$ & $\mathrm{~N}$ & $\mathrm{H}$ & $\mathrm{H}$ & $\mathrm{N}$ & $\mathrm{H}$ & $\mathrm{H}$ \\
\hline 8 & $\mathrm{~N}$ & $\mathrm{H}$ & $\mathrm{H}$ & $\mathrm{H}$ & $\mathrm{H}$ & $\mathrm{H}$ & $\mathrm{H}$ & $\mathrm{H}$ \\
\hline 9 & $\mathrm{~N}$ & $\mathrm{~N}$ & $\mathrm{~N}$ & $\mathrm{~N}$ & $\mathrm{H}$ & $\mathrm{H}$ & $\mathrm{H}$ & $\mathrm{H}$ \\
\hline 10 & $\mathrm{H}$ & $\mathrm{H}$ & $\mathrm{H}$ & $\mathrm{H}$ & $\mathrm{H}$ & $\mathrm{H}$ & $\mathrm{H}$ & $\mathrm{H}$ \\
\hline 11 & $\mathrm{H}$ & $\mathrm{N}$ & $\mathrm{UH}$ & $\mathrm{H}$ & $\mathrm{H}$ & $\mathrm{UH}$ & $\mathrm{UH}$ & $\mathrm{H}$ \\
\hline 12 & $\mathrm{H}$ & $\mathrm{H}$ & $\mathrm{N}$ & $\mathrm{H}$ & $\mathrm{H}$ & $\mathrm{UH}$ & $\mathrm{UH}$ & $\mathrm{N}$ \\
\hline 13 & $\mathrm{H}$ & $\mathrm{H}$ & $\mathrm{H}$ & $\mathrm{H}$ & $\mathrm{H}$ & $\mathrm{UH}$ & $\mathrm{UH}$ & $\mathrm{N}$ \\
\hline 14 & $\mathrm{~N}$ & $\mathrm{H}$ & $\mathrm{H}$ & $\mathrm{H}$ & $\mathrm{H}$ & $\mathrm{H}$ & $\mathrm{H}$ & $\mathrm{H}$ \\
\hline 15 & $\mathrm{~N}$ & $\mathrm{H}$ & $\mathrm{H}$ & $\mathrm{H}$ & $\mathrm{H}$ & $\mathrm{H}$ & $\mathrm{H}$ & $\mathrm{H}$ \\
\hline
\end{tabular}

\subsection{One-way Analysis of Variance (ANOVA)}

One-way ANOVA was conducted to determine if the change detected in $\mathrm{HR}, \mathrm{SC}$, arousal and valence was significant. One-way ANOVA examines the difference between groups that are classified by one independent variable.

First one-way ANOVA was conducted on HR results. That was done by entering the mean HR values to MINITAB software. Mean HR under white light was chosen as the base and oneway ANOVA test was run on the data. The results are tabulated and shown in Table 4.12. DF in the table denotes the degrees of freedom; it refers to the number of independent observations that are calculated in the sum of squares. SS denotes sum of squares, which tells how much of the total variability is explained by the experimental effect. MS represents the mean square, which is sum of squares (SS) divided by its associated degrees of freedom (DF). P-value is the significance level.

The same steps as above were followed to run one-way ANOVA on SC values. The results are tabulated and shown in Table 4.13.

It is observed from Table $4.12 \&$ Table 4.13 that the $p$-value is very high under all colour lights. This means that the variation in HR and SC in response to the change in the colour and 
Table 4.12: One-way ANOVA for HR

\begin{tabular}{|c|c|c|c|c|c|}
\hline Coloured light & DF & SS & MS & F & P \\
\hline Blue-low & 1 & 24.2 & 24.2 & 0.60 & 0.44 \\
\hline Blue-high & 1 & 5.3 & 5.3 & 0.12 & 0.73 \\
\hline Green-low & 1 & 0.1 & 0.1 & 0.00 & 0.97 \\
\hline Green-high & 1 & 2.0 & 2.0 & 0.05 & 0.83 \\
\hline Red-low & 1 & 0.3 & 0.3 & 0.01 & 0.93 \\
\hline Red-high & 1 & 14.0 & 14.0 & 0.34 & 0.57 \\
\hline Natural & 1 & 50.3 & 50.3 & 1.08 & 0.31 \\
\hline
\end{tabular}

Table 4.13: One-way ANOVA for SC

\begin{tabular}{|c|c|c|c|c|c|}
\hline Coloured light & DF & SS & MS & F & P \\
\hline Blue-low & 1 & 67 & 67 & 0.17 & 0.69 \\
\hline Blue-high & 1 & 184 & 184 & 0.46 & 0.51 \\
\hline Green-low & 1 & 522 & 522 & 1.10 & 0.30 \\
\hline Green-high & 1 & 824 & 824 & 1.36 & 0.25 \\
\hline Red-low & 1 & 697 & 697 & 1.13 & 0.26 \\
\hline Red-high & 1 & 438 & 438 & 0.85 & 0.37 \\
\hline Natural & 1 & 696 & 696 & 1.38 & 0.25 \\
\hline
\end{tabular}

intensity of light is not significant when considering all participants under each coloured light.

The one-way ANOVA results for arousal and valence were also tabulated and are shown in Table 4.14 \& Table 4.15.

It is observed from Table 4.14 that the p-value was very high under all coloured lights except for red high light where $p=0.04$. This means that the change in arousal in response to the change in the colour and intensity of light is significant when considering the average arousal effect off all participants under red high light. However it is not significant under all other colour lights.

It is observed from Table 4.15 that the p-value was very high under all coloured lights except for green high light where $p=0.03$. This again means that the change in valence in response to the change in the colour and intensity of light is significant when considering the average valence effect off all participants under green high light. However it is not significant under all other colour lights. 
Table 4.14: One-way ANOVA for arousal

\begin{tabular}{|c|c|c|c|c|c|}
\hline Coloured light & DF & SS & MS & F & P \\
\hline Blue-low & 1 & 1.20 & 1.20 & 0.27 & 0.61 \\
\hline Blue-high & 1 & 9.63 & 9.63 & 2.05 & 0.16 \\
\hline Green-low & 1 & 0.53 & 0.53 & 0.13 & 0.72 \\
\hline Green-high & 1 & 4.80 & 4.80 & 1.43 & 0.24 \\
\hline Red-low & 1 & 7.50 & 7.50 & 2.42 & 0.13 \\
\hline Red-high & 1 & 16.13 & 16.13 & 4.46 & 0.04 \\
\hline Natural & 1 & 1.20 & 1.20 & 0.20 & 0.66 \\
\hline
\end{tabular}

Table 4.15: One-way ANOVA for valence

\begin{tabular}{|c|c|c|c|c|c|}
\hline Coloured light & DF & SS & MS & F & P \\
\hline Blue-low & 1 & 0.13 & 0.13 & 0.04 & 0.84 \\
\hline Blue-high & 1 & 1.20 & 1.20 & 0.48 & 0.50 \\
\hline Green-low & 1 & 3.33 & 3.33 & 1.54 & 0.23 \\
\hline Green-high & 1 & 12.03 & 12.03 & 5.06 & 0.03 \\
\hline Red-low & 1 & 0.13 & 0.13 & 0.04 & 0.83 \\
\hline Red-high & 1 & 0.00 & 0.00 & 0.00 & 1.00 \\
\hline Natural & 1 & 9.63 & 9.63 & 3.56 & 0.07 \\
\hline
\end{tabular}

\subsection{Two-way Analysis of Variance (ANOVA)}

Two-way ANOVA was conducted to determine if the change in $\mathrm{HR}, \mathrm{SC}$, arousal and valence are significant when considering each participant separately. Two-way ANOVA is used to analyse the effect of two factors on one dependent variable. The two factors considered were 'person' and 'colour'. The variable was HR, SC, arousal or valence. When considering person as a factor, the significance of the variable was tested for each participant separately. When considering colour as a factor, the significance of the variable was tested for all participants under a certain colour and intensity light.

Two-way ANOVA test was conducted by entering the mean HR, SC, arousal and valence data in MINITAB software. The two factors were chosen then the test was run on each set of data (HR, SC, arousal and valence). The results for each set of data were then tabulated. The results for two-way ANOVA for HR and SC are shown in Table 4.16, Table 4.17.

It is observed from Tables $4.16 \& 4.17$ that although the change in mean HR and SC was 
not significant when considering all participants under each colour light, it was very significant when considering each participant separately.

Table 4.16: Two-way ANOVA for HR

\begin{tabular}{|c|c|c|c|c|c|}
\hline Factor & DF & SS & MS & F & P \\
\hline Person & 14 & 2564 & 183.1 & 8.52 & 0.00 \\
\hline Colour & 7 & 190.8 & 27.26 & 1.27 & 0.27 \\
\hline
\end{tabular}

Table 4.17: Two-way ANOVA for SC

\begin{tabular}{|c|c|c|c|c|c|}
\hline Factor & DF & SS & MS & F & P \\
\hline Person & 14 & 51702 & 3693 & 19.53 & 0.00 \\
\hline Colour & 7 & 1452.7 & 207.5 & 1.10 & 0.37 \\
\hline
\end{tabular}

The results of two-way ANOVA for arousal and valence data are shown in in Tables 4.18 \& 4.19.

It is observed from Tables $4.18 \& 4.19$ that the change in arousal and valence is significant when considering the change of all subjects under each colour light and when considering the change for each participant separately.

Table 4.18: Two-way ANOVA for arousal

\begin{tabular}{|c|c|c|c|c|c|}
\hline Factor & DF & SS & MS & F & P \\
\hline Person & 14 & 203.3 & 14.52 & 4.33 & 0.00 \\
\hline Colour & 7 & 71.2 & 10.17 & 3.04 & 0.01 \\
\hline
\end{tabular}

Table 4.19: Two-way ANOVA for valence

\begin{tabular}{|c|c|c|c|c|c|}
\hline Factor & DF & SS & MS & F & P \\
\hline Person & 14 & 99.22 & 7.09 & 3.30 & 0.00 \\
\hline Colour & 7 & 39.60 & 5.66 & 2.63 & 0.02 \\
\hline
\end{tabular}


CHAPTER 5

Discussion

7 his research is built on the current body of knowledge that physiological and psycho-

ـ logical measures are necessary to measure the effect of light intensity and colour on space users. The thesis reports changes in HR and SC and SAM of participants when exposed to different colour and intensity lightings for periods of time that allows them to adapt to the conditions.

Observation 1: It is observed from Table 4.2 that there is variation in HR under different colour and intensity lights.

Discussion 1: It is well documented in literature that there is a close correlation between HR and emotion. This observation directly confirms that change of colour and intensity of lights in the space causes a change in the HR which is due to the change in the emotions and the performance of people.

Observation 2: It is observed from Table 4.2 that the inter subject variation in HR is very high.

Discussion 2: This demonstrates that people have different HR under normal conditions, which makes it difficult to compare their HR. This is the reason why HR data needs to be normalized. 
Observation 3: It is observed from Table 4.3 \& Table 4.4 that the magnitude of heart rate variability (HRV) was also subject dependent.

Discussion 3: It is well documented in literature that $\mathrm{HRV}$ is a good measure of emotions. This observation demonstrates that HRV is not a good measure of the effect of a stimulus on a group of people because the magnitude of change in HRV differs between people, which make it difficult to compare their HRV.

Observation 4: The direction of change in HR (increase or decrease) is subject dependent, where the same colour and intensity light can cause and increase in HR for some subjects and a decrease in HR for others.

Discussion 4: This observation demonstrates that it is not possible to correlate the colour and lighting conditions to a specific effect on all participants. This confirms what Veitch reported that successful lighting in one condition might prove impractical in another because of a number of constrains that include individual differences [19].

Observation 5: Some colour and intensity conditions caused greater changes in HR than others. Red-high light caused the greatest impact on the HR of participants where it caused the largest increase in HR of $20 \%$ of the participants and the largest decrease in HR of $20 \%$ of the participants. Blue-low and natural lights also caused a large impact on the HR of participant.

Discussion 5: This observation demonstrates that some colour and intensity lights have greater impact on emotions than others. HR results show that Red-light had the greatest impact on the emotions of participants.

Observation 6: It is observed from Table 4.5 that there is a change in SC of participants when they are exposed to different colour and intensity lights.

Discussion 6: It is well documented in literature that there is a close correlation between SC and emotion. This observation directly confirms that the change in the colour and intensity of lights in the space causes a change in SC which is due to the change in the emotions and performance of users. 
Observation 7: It is observed from Table 4.5 that the inter subject variation in SC is very high.

Discussion 7: This demonstrates that people have different SC under normal conditions, which makes it difficult to compare their SC. This is the reason why SC data needs to be normalized.

Observation 8: It is observed from Table 4.6 \& Table 4.7 that the change in SC was also subject dependent where the magnitude of change in SC differs between people.

Discussion 8: This demonstrates that it is difficult to use SC as a measure of the effect of a stimulus on a group of people because the inter subject variation is very high.

Observation 9: It is observed that the direction of change in SC (increase or decrease) is subject dependent.

Discussion 9: This observation again proves (as mentioned in discussion 2) that it is not possible to correlate the colour and lighting condition to a specific effect on all participants. This is because the same colour and lighting condition can have different emotional effects on different people.

Observation 10: $60-73 \%$ of the non-White conditions caused higher SC than White conditions.

Discussion 10: In most experiments (2/3 of the experiments) White light caused the lowest SC. Since SC is associated with the arousal of the participants, White light caused the most relaxing conditions for most participants.

Observation 11: Some colour and intensity lights caused greater changes in SC than others. Natural light caused the greatest impact on SC of participants. Green-high light and Red-high light also caused a big impact on the SC of participants.

Discussion 11: This observation again demonstrates that some colour lights affect emotions more than others. From observations 2 and 6 it is observed that Natural light and Red-high 
light caused the greatest impact on the HR and SC of participants, therefore they were the most mood inducing conditions.

Observation 12: It is observed from Table 4.8 \& Table 4.10 that there is a change in arousal and valence rating under different colour and intensity lights.

Discussion 12: The above observation demonstrates that the lighting colour and intensity of the space affects the arousal and valence of the users. Arousals shows how excited or relaxed the participants are, valence shows how happy or unhappy they are, therefore the above confirms that light and colour affect the mood and performance of the space users.

Observation 13: It is observed from Table 4.8 \& Table 4.10 that the changes in the arousal and valence of participants were subject dependent.

Discussion 13: This observation confirms what has been demonstrated in discussions 3 and 5 that it is not possible to correlate the colour and lighting condition to a specific effect on all participants. This is because the same colour and lighting condition can have different arousal and valence effects on different people.

Observation 14: It is observed from Table 4.9 that although changes in arousal were subject dependent, general effects for some colour lights were seen. White light caused a neutral effect on $67 \%$ of participants; Blue-low light caused a relaxing condition for $60 \%$ of participant and Blue-high light caused that effect for $73 \%$ of participants. Red-low light caused an exciting effect for $53 \%$ of participants and Red-high light caused that effect for $60 \%$ of participants.

Discussion 14: Although it is difficult to correlate colour and lighting conditions to a specific effect on all participants, some general effects were drawn from the results. White light caused a neutral effect for $67 \%$ of the participants, Blue-lights caused a relaxing effect for $60-73 \%$ of participants and Red-lights caused an exciting effect for $53-60 \%$ of participants.

Observation 15: It is observed from Table 4.11 that all colour lights caused a happy or neutral effect on most participants. Green-high light caused the largest pleasant effect; it 
caused a happy effect for $93 \%$ of participants. Green-low light caused the same effect for $80 \%$ of participants while natural light caused that effect for $73 \%$ of participants. The most unpleasant effects were seen under Red-high light, which caused an unhappy effect on $26 \%$ of participants, Blue-high and Red-low caused that effect on $20 \%$ of participants.

Discussion 15: Although it is not possible to correlate the colour and lighting conditions to a specific effect on all participants, some general effects were drawn from the results. Green lights caused the most pleasant effects where they had a happy effect on a large number of participants (80-93\%) and Natural light caused the same effect for $73 \%$ of the participants. The observed unpleasant effects were less correlated and the most unpleasant effects recorded were under red lights that caused an unhappy effect on $20-26 \%$ of the participants.

Observation 16: From Table 4.16 \& Table 4.17 it is observed that the changes in HR and SC are not significant when considering all participants under each colour and intensity light due to the large inter subject variation; however the changes are very significant when considering each participant individually.

Discussion 16: This confirms that people have different HR and SC in normal conditions. This makes the use of HR and SC to measure the effect of a stimulus on a group of people is unreliable because it is difficult to compare the results as the inter subject variation will be very high. This observation also demonstrates that although the colour and lighting conditions had different effects on participants, they caused a significant change in HR and SC of all participants. This confirms that colour and intensity of light has a significant effect on peoples' emotions.

Observation 17: It is observed from Table 4.18 \& Table 4.19 that the change in arousal and valence is significant when considering the change of all subjects and each subject separately.

Discussion 17: This observation demonstrates that although the colour and lighting conditions have different effects on participants, there was a significant change in arousal and valence for all participants in response to change in the colour and lighting conditions. This confirms that colour and lighting conditions cause a significant change in emotions of people. 
Observation 18: It is observed from the results that there is no correlation between the physiological measures (HR and SC) and the psychological measures (valence and arousal).

Discussion 18: It is reported in literature that HR is related to valence and SC is a useful measure of arousal [21]. However the results of this study show no co-relation between the physiological measures (HR and SC) and the psychological measures (valence and arousal). This could be due to a number of reasons.

Bradley et.al. reports that different measures differ greatly in sensitivity and dynamic range. While evaluation language has enormous sensitivity and extension, autonomically mediated responses (e.g., heart rate and skin conductance) vary little with stimulus changes that are rated in the lower range of affective intensities [21]. The difference in the sensitivity of the measures could be the reason for not finding co-relation between physiological and psychological measures.

It is also reported by Detenber et.al. that self reports and physiological measures tap different systems and index different facets of emotion. Though they are often correlated, they do not necessarily need to be. An example of that would be some phobics who report extremely high levels of emotion when confronted with the object of their fears or asked to imagine it, yet their physiological responses do not reflect the terror they experience [1]. While physiological and psychological measures should generally converge, situations exist where they do not. The results of this study represent one of these situations.

Another possible reason for the difference seen in the physiological and psychological measures is people believing that lights and colours affect them in certain ways. These beliefs could be influenced by culture or social context. However the impact of these beliefs might be limited to the conscious experience of emotion (i.e. self reports).

\subsection{Summary of Discussion}

This research demonstrates that there is a change in the physiological measures (HR and SC) due to change in the colour and intensity of lights in the space. However the change is subject dependent, which makes it difficult to correlate the colour and lighting conditions to a specific effect on all participants. This means that it is difficult to predict a specific effect for the colour 
and light of the space on all the users, therefore to study the effect of colour and light in the space the individual differences of users must be taken into account. This directly confirms what Veitch et.al. reported that successful lighting in one situation might prove impractical in another because of a number of constrains, one of them is individual differences [19].

It was also seen that some colour and intensity lights has a greater impact on HR and SC than others. Natural light and Red-high light caused the greatest impact on SC and HR. SC results show that White light caused the most relaxing condition for $60-73 \%$ of the participants.

Although it is well documented in literature that HR, HRV and SC are a good measure of emotion, this research demonstrates that they are not a good measure of the emotions of a group of people. The inter subject variation in HR, HRV and SC is very high which makes their use to measure the effect of a stimulus on a group of people unreliable because it is difficult to compare the results.

The subjective tests demonstrate that there was a change in arousal and valence due to change in colour and intensity of lights in the space; however the change was again subject dependent. It is also seen that some colour and intensity lights had a greater impact on the arousal and valence of participants than others.

Although it is difficult to correlate the colour and lighting conditions to a specific effect on all users, some general effects were drawn from the arousal and valence results. White light caused a neutral effect for $67 \%$ of the participants; Blue lights caused a relaxing effect for $60-73 \%$ of participants and Red lights caused an exciting effect for $53-60 \%$ of participants. Green lights caused a pleasant effect on $80-93 \%$ of the participants; Natural light caused the same effect for $73 \%$ of the participants. The most unpleasant effects recorded were under red lights, which caused an unhappy effect on $20-26 \%$ of the participants.

It has been reported by Knez that the colour and type of lamp of indoor lighting may have different emotional meaning to different age groups and gender [47]. However, Valdez et.al. report that emotional reactions to colour were similar for men and women [24]. The impact of light and colour on different age groups and gender are not taken into account in this research.

Veitch reported that in studies with long adaptation time, there might be no relationship between illumination and performance [19]. This might suggest that with longer exposure to lights there would not be a relationship between illumination and emotions. This research studies the impact of light and colour on people when they are exposed to each condition for 10 minutes; 
preliminary experiments demonstrated that this time was sufficient to allow participants to adapt to the conditions. It is recommended that further research would study the effect of longer exposure to light and colour.

Further research is also recommended to study the relation ship between colour preferences of space users and the effect of colour and light on them, also studying a larger sample might help in finding general effects for some colours. 
CHAPTER 6

\section{Conclusion}

his research studies the emotional effects of the colour and intensity of lights on space
users. It is reported in literature that emotion manifests itself in three separate sections (i) physiological (i.e. objective measures), (ii) psychological (i.e. subjective experience) and (iii) behavioural, and it is necessary that studies not be limited to the assessment of a single response. Therefore it was important to take both objective and subjective measures into account when measuring the emotional effects of light and colour on people. This research reports the changes in physiological measures (HR and SC) and psychological measures (SAM) due to change in the colour and intensity of lights in the space.

The experiments were conducted on 15 participants that were exposed to 8 different colour and intensity lights. Their SC and HR were recorded during the experiment and they were asked to complete SAM after being exposed to each colour and intensity light.

This research demonstrates that there is a change in the physiological measures ( $\mathrm{HR}$ and SC) due to change in the colour and intensity of lights in the space. However the changes are subject dependent, which makes it hard to correlate the colour and lighting conditions to a specific effect on all participants. This means that it is hard to predict a specific effect for colour and lighting of the space on all the users. The research suggests that architects and designers of any space must take into account the individual differences of the predicted users when designing the colours and lights.

The results also demonstrate that some colour and intensity lights had a greater impact on 
HR and SC than others. Natural light and Red-high light caused the greatest impact on SC and HR. SC results show that White light caused the most relaxing condition for $60-73 \%$ of the participants.

Although it is well documented in literature that HR, HRV and SC are a good measure of emotion, this research demonstrates that they are not a good measure of the emotions of a group of people. The inter subject variation in HR, HRV and SC is very high which makes their use to measure the effect of a stimulus on a group of people unreliable because it is hard to compare the results.

The psychological tests demonstrate that there was a change in arousal and valence due to change in colour and intensity of lights in the space; however the changes are subject dependent. It is also seen that some colour and intensity lights had a greater impact on the arousal and valence of participants than others.

It is also observed from the results that there is no co-relation between the physiological and psychological measures.

Although it is hard to correlate the colour and lighting conditions to a specific effect on all users, some general effects are drawn from the results. White light caused a neutral effect for $73 \%$ of the participants; Blue lights caused a relaxing effect for $60-73 \%$ of participants and Red lights caused an exciting effect for $53-60 \%$ of participants. Green lights caused a pleasant effect on $80-93 \%$ of the participants; Natural light caused the same effect for $73 \%$ of the participants. The most unpleasant effects recorded were under red lights, which caused an unhappy effect on $20-26 \%$ of the participants.

Future research: It is recommended that future research would study the following:

1. The impact of light and colour on different age groups and gender.

2. The effect of longer exposure to light and colour.

3. The relationship between colour preferences of space users and the effect of colour and light on them.

4. Study a larger sample, which might help in finding general effects for some colours. 
APPENDIX A

ECG Recordings 


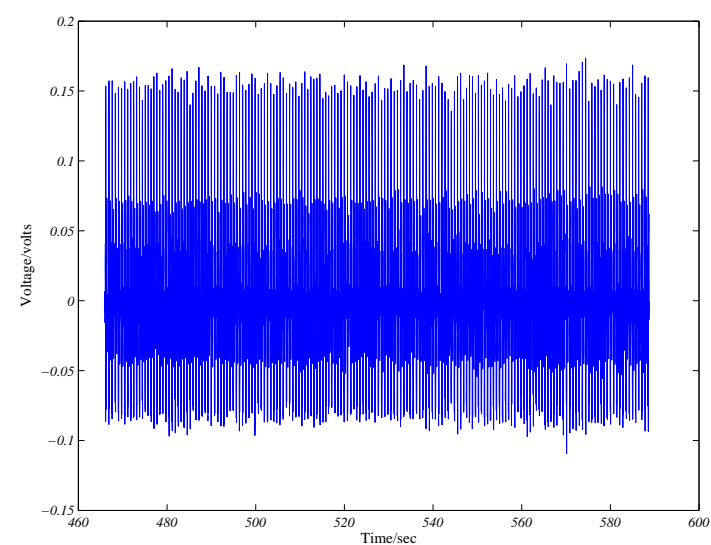

(a) White light

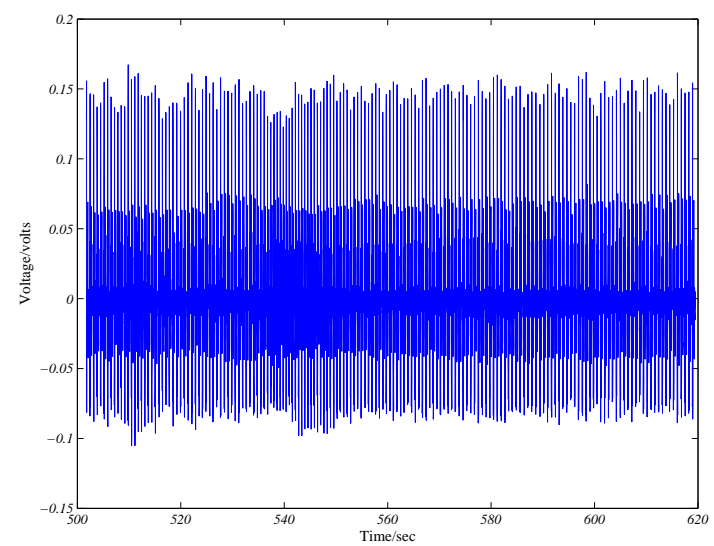

(c) Blue-high light

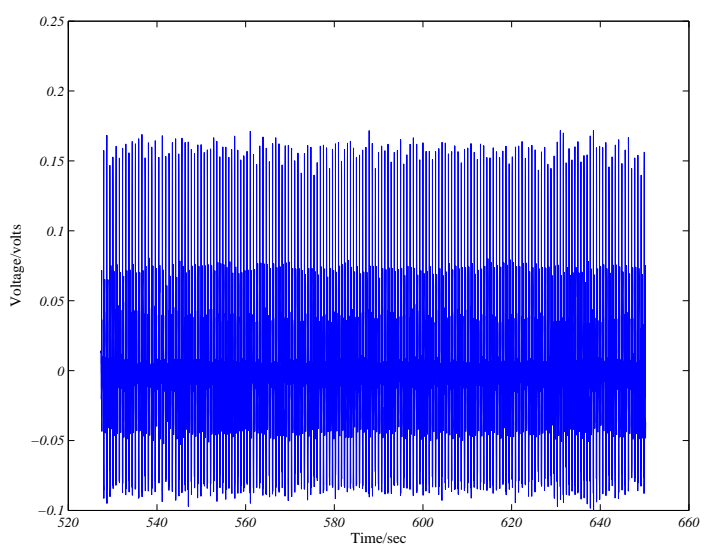

(b) Blue-low light

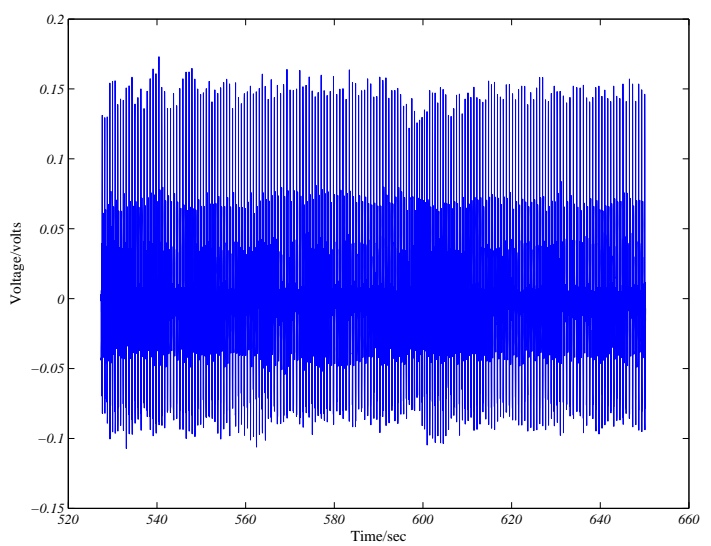

(d) Green-low light

Figure A.1: Participant 1 - ECG Recordings for participant 1 for all colour lights 


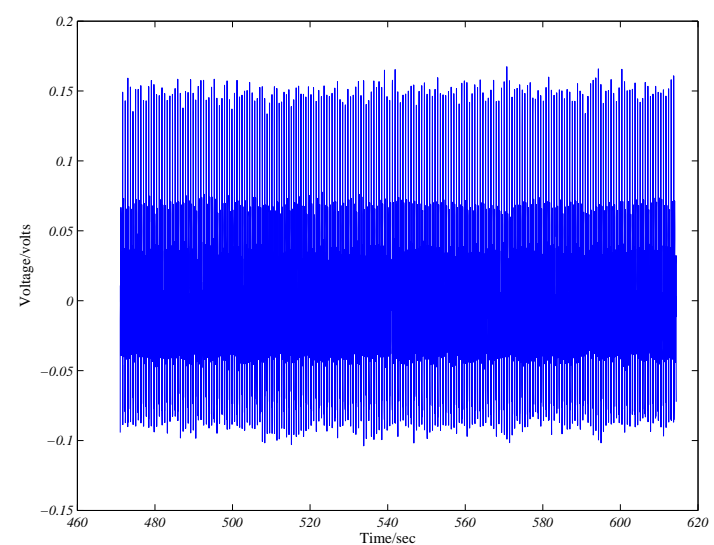

(e) Green-high light

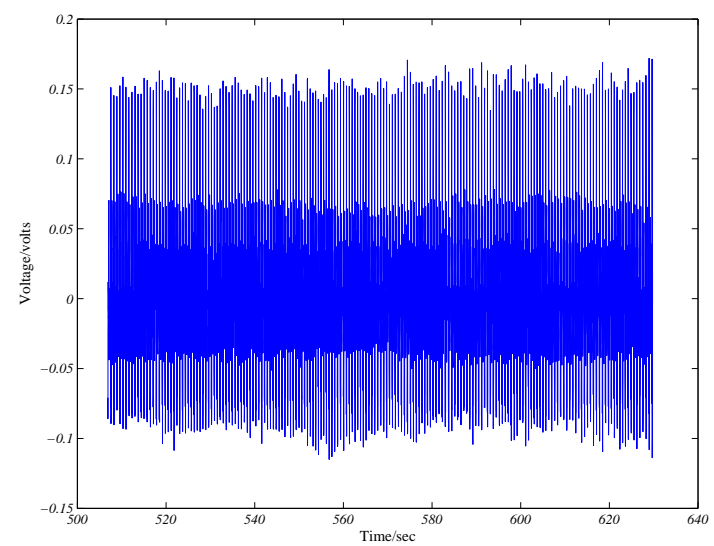

(g) Red-hig light

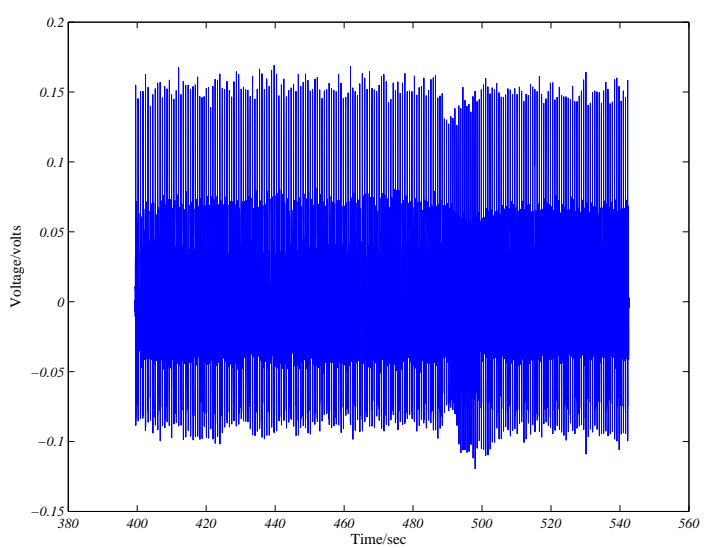

(f) Red-low light

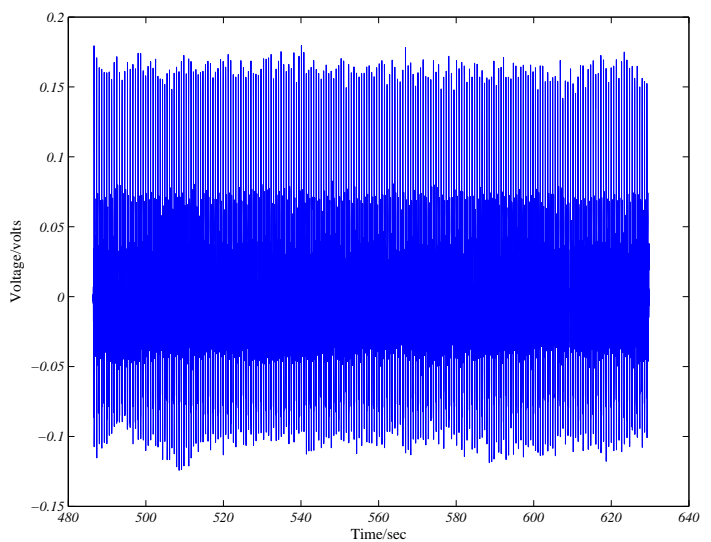

(h) Natural light

Figure A.1: Participant 1 - ECG Recordings for participant 1 for all colour lights 


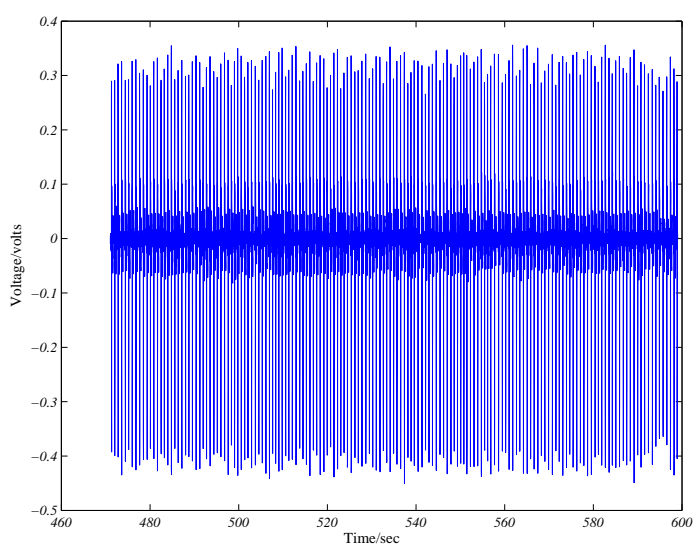

(a) White light

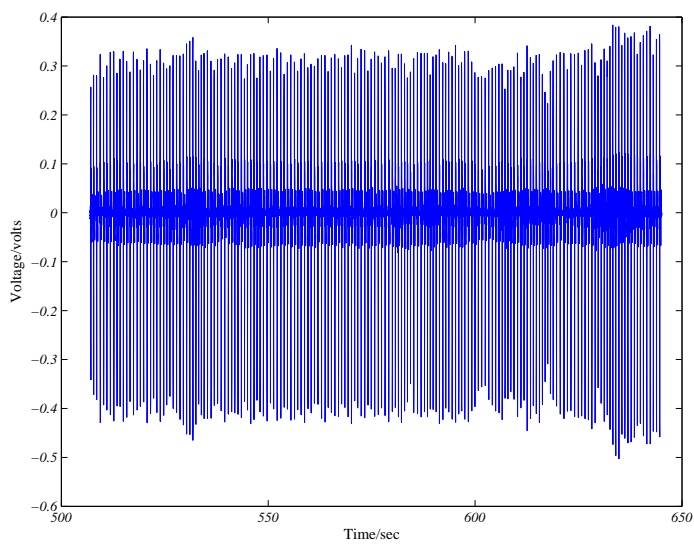

(c) Blue-high light

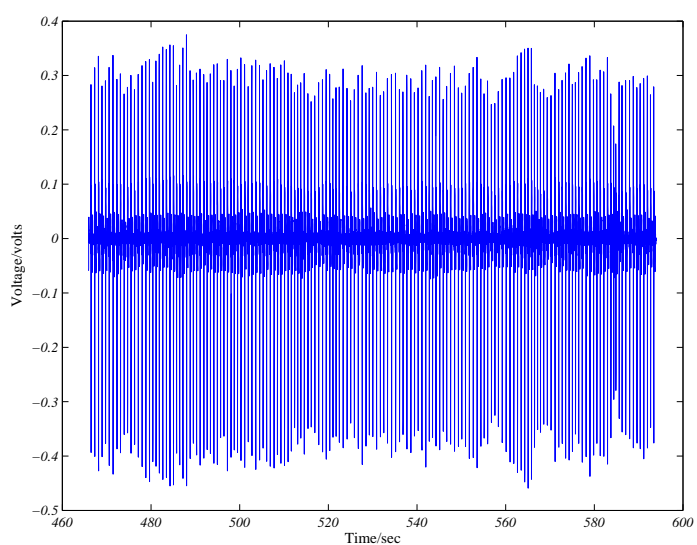

(b) Blue-low light

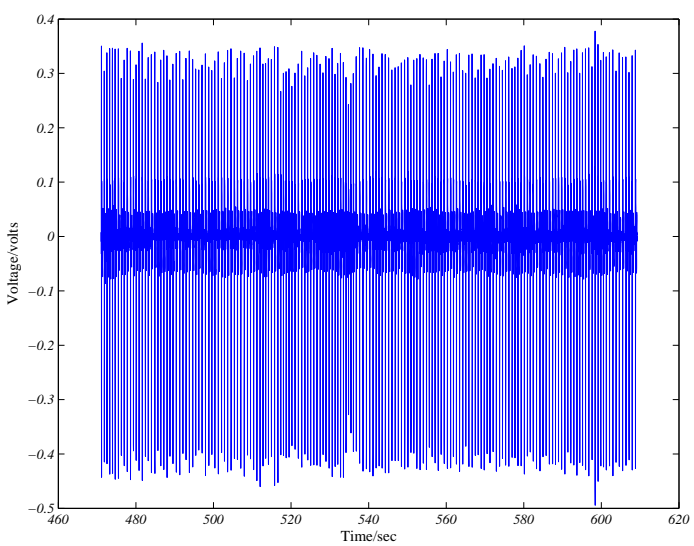

(d) Green-low light

Figure A.2: Participant 2 - ECG Recordings for participant 2 for all colour lights 


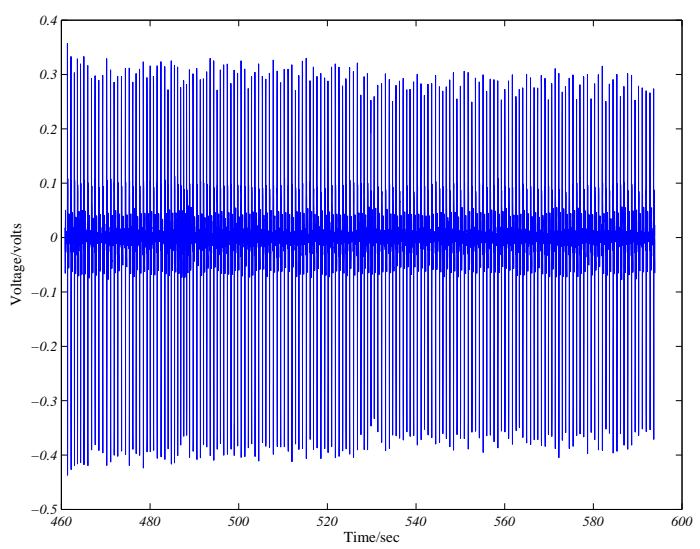

(e) Green-high light

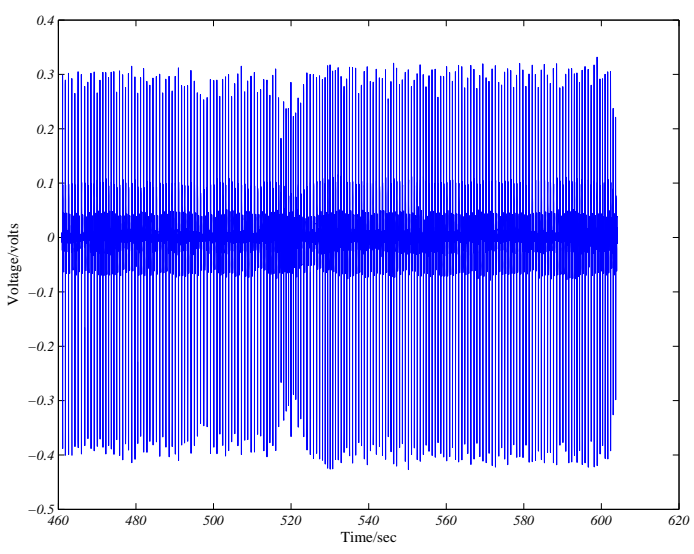

(g) Red-hig light

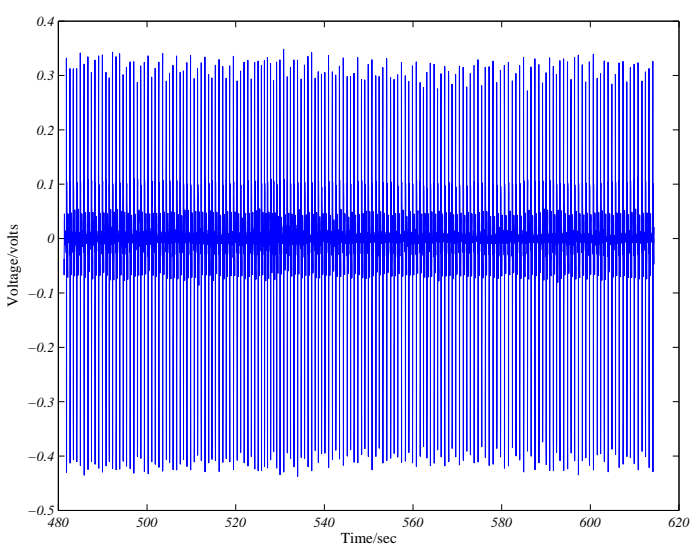

(f) Red-low light

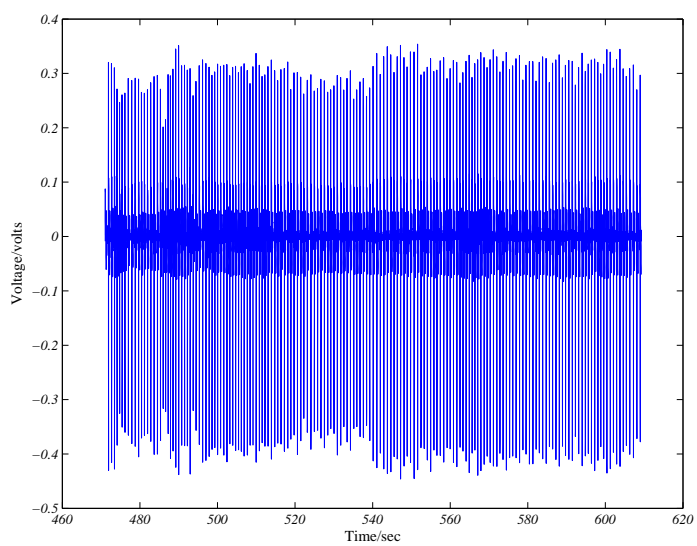

(h) Natural light

Figure A.2: Participant 2 - ECG Recordings for participant 1 for all colour lights 


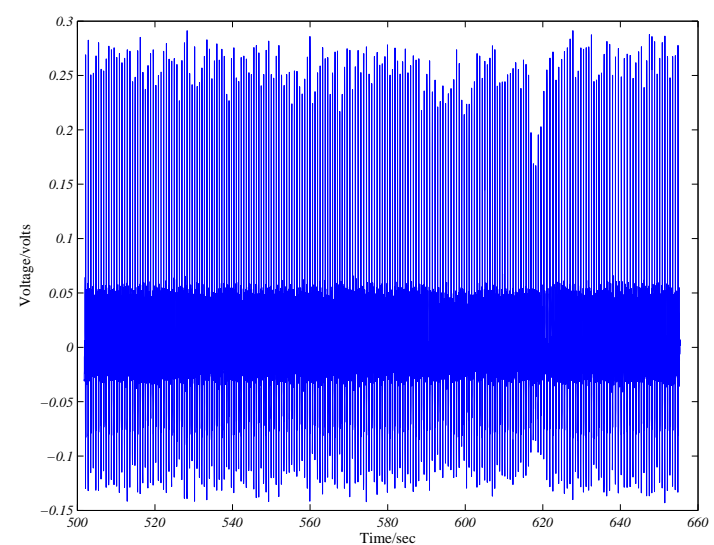

(a) White light

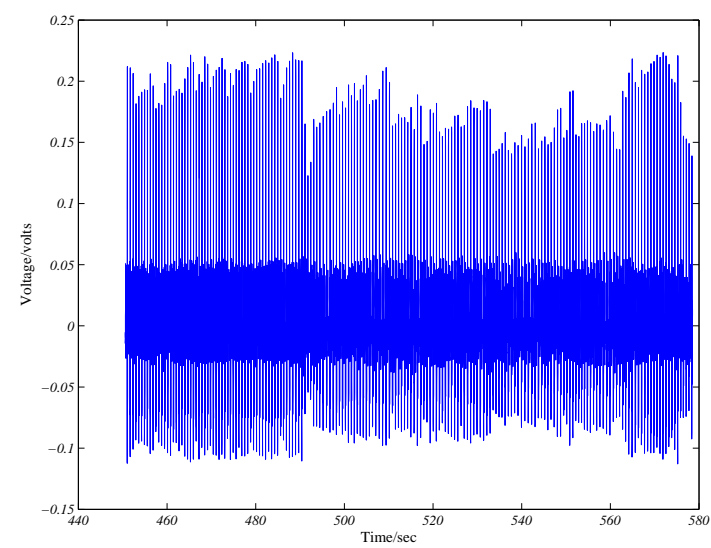

(c) Blue-high light

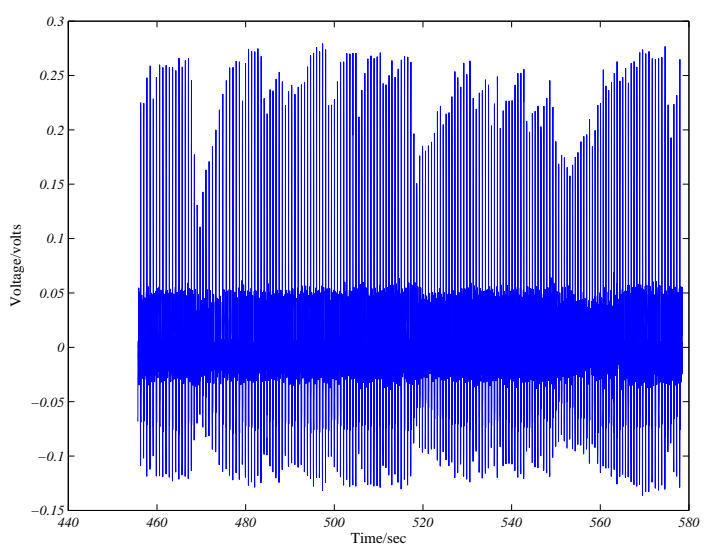

(b) Blue-low light

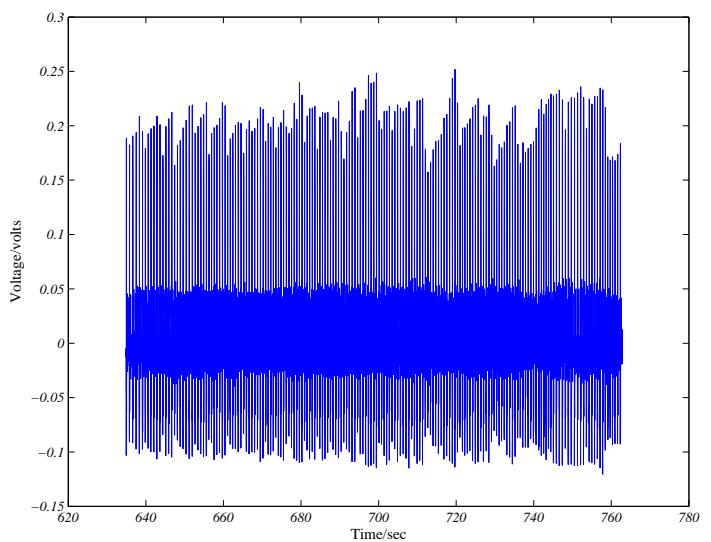

(d) Green-low light

Figure A.3: Participant 3 - ECG Recordings for participant 3 for all colour lights 


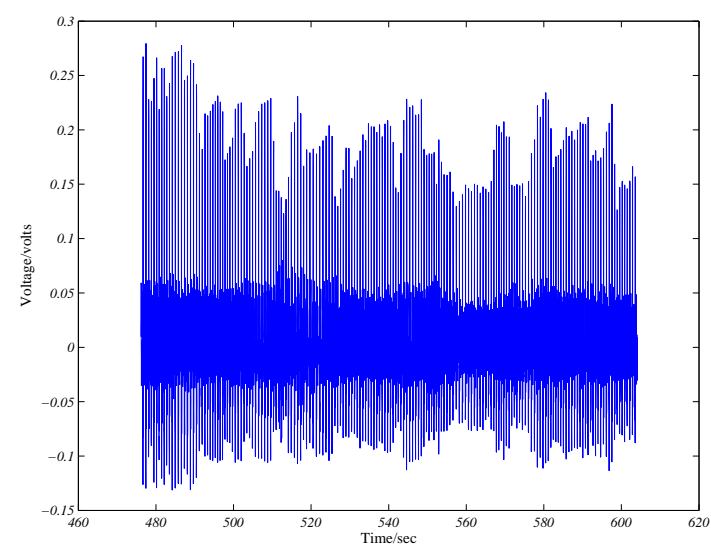

(e) Green-high light

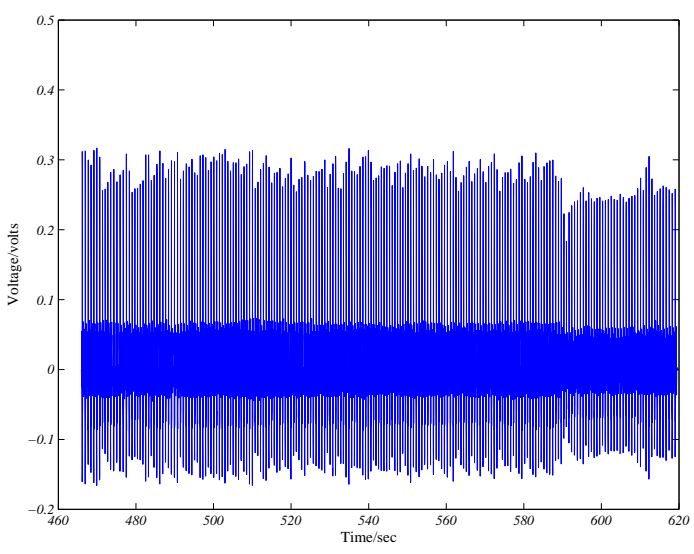

(g) Red-hig light

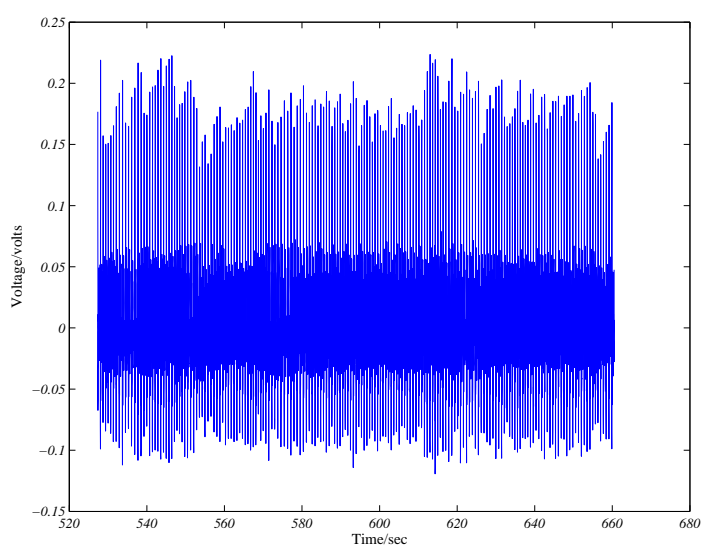

(f) Red-low light

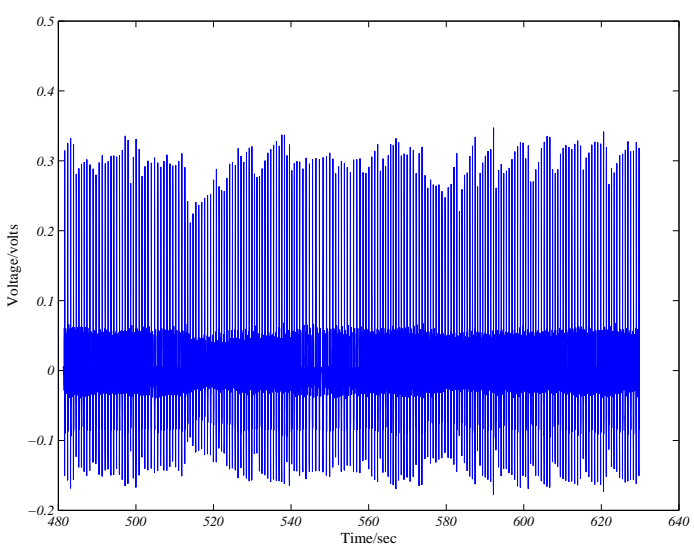

(h) Natural light

Figure A.3: Participant 3 - ECG Recordings for participant 3 for all colour lights 


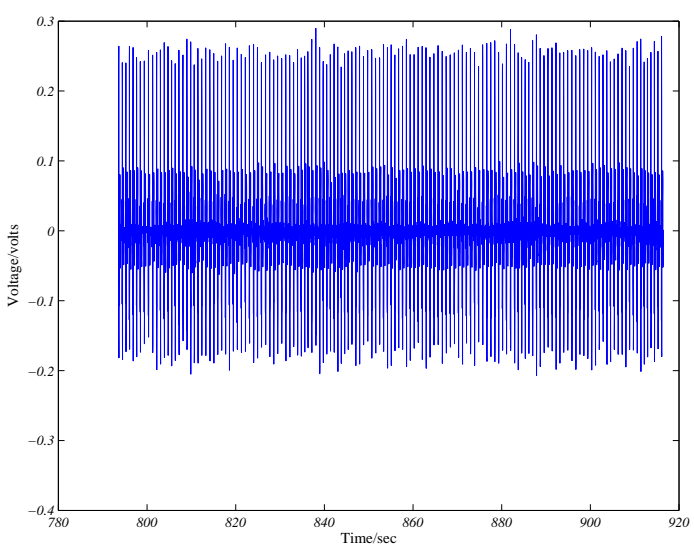

(a) White light

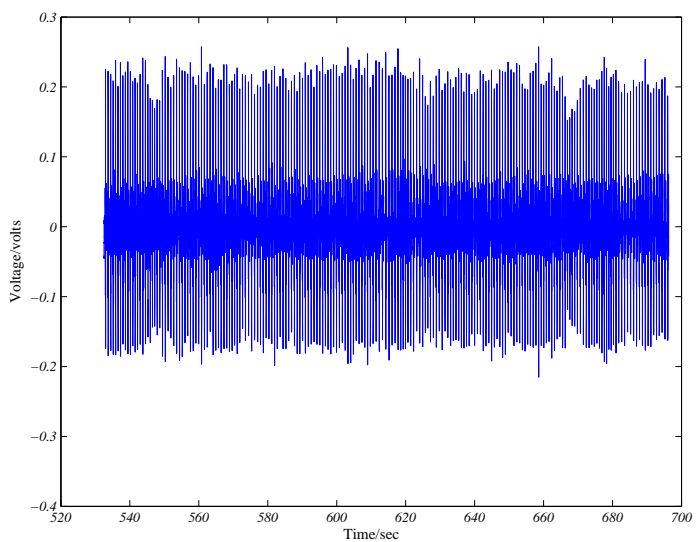

(c) Blue-high light

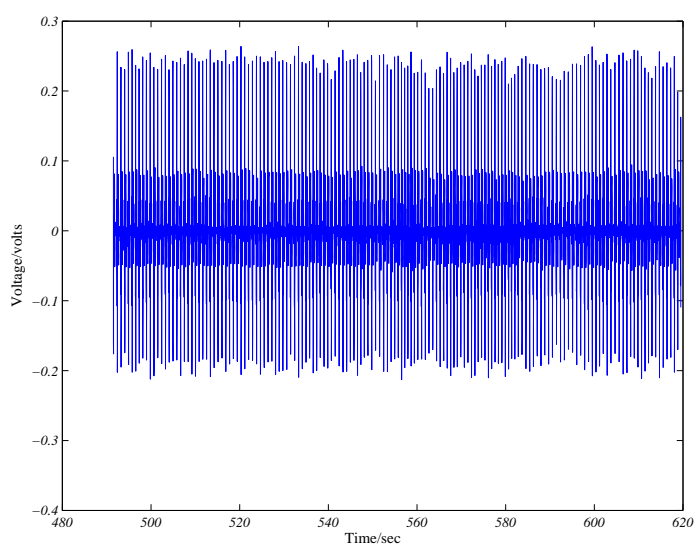

(b) Blue-low light

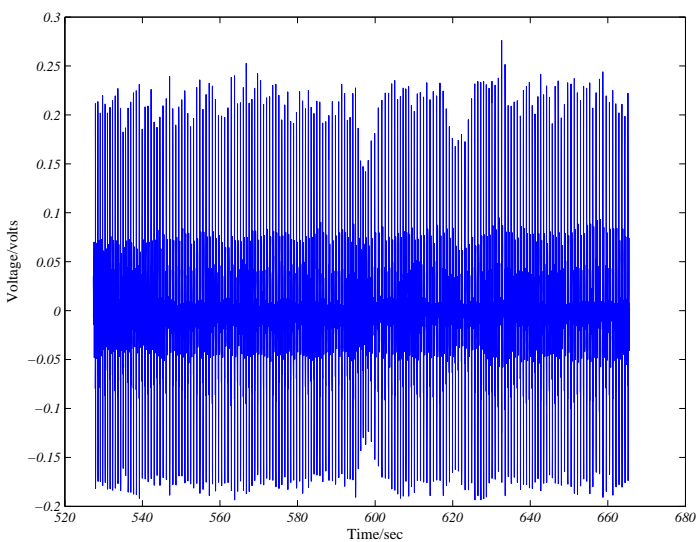

(d) Green-low light

Figure A.4: Participant 4 - ECG Recordings for participant 4 for all colour lights 


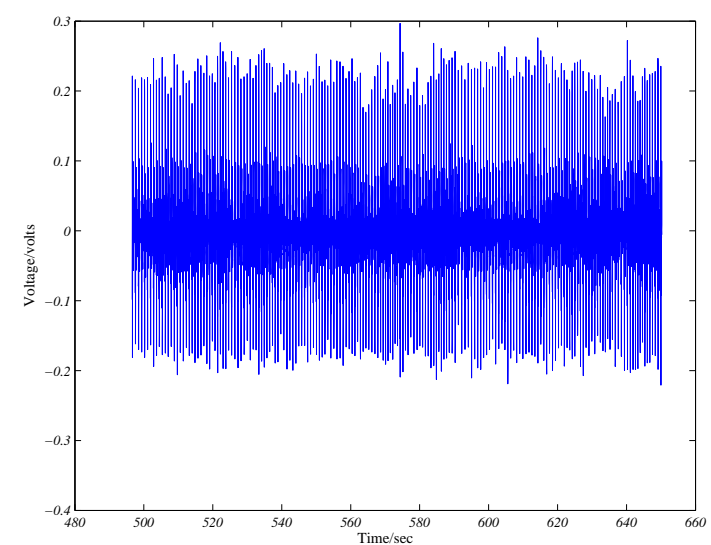

(e) Green-high light

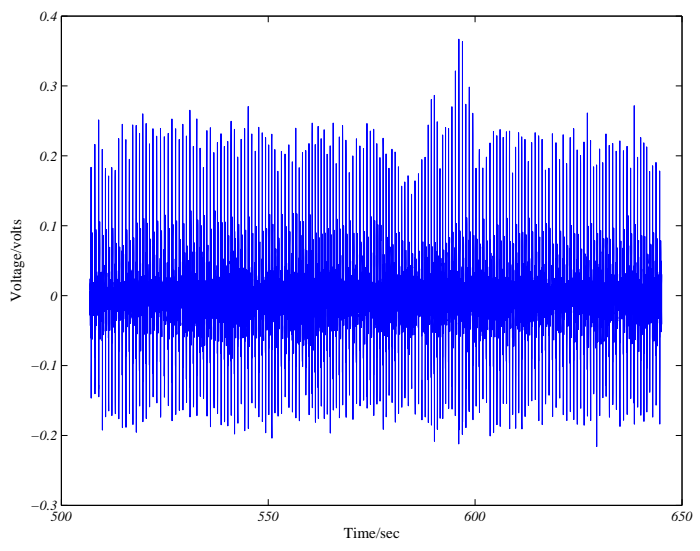

(g) Red-hig light

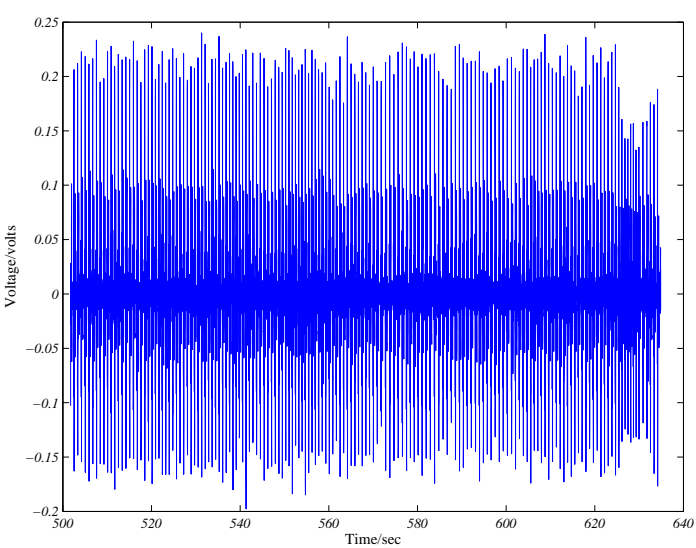

(f) Red-low light

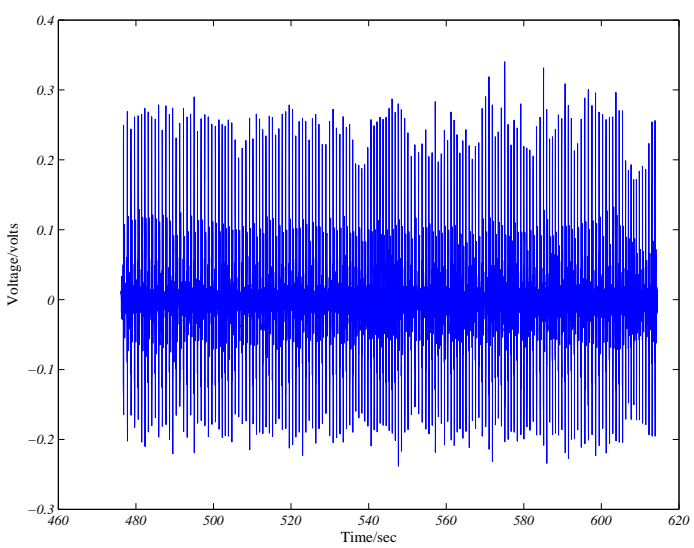

(h) Natural light

Figure A.4: Participant 4 - ECG Recordings for participant 4 for all colour lights 


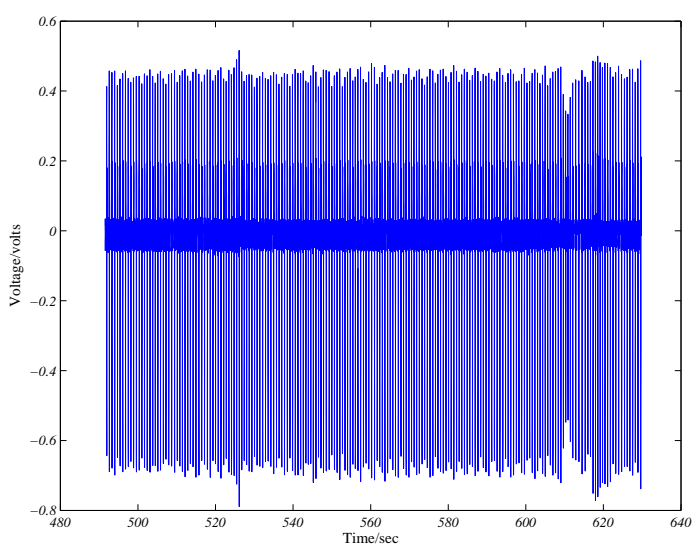

(a) White light

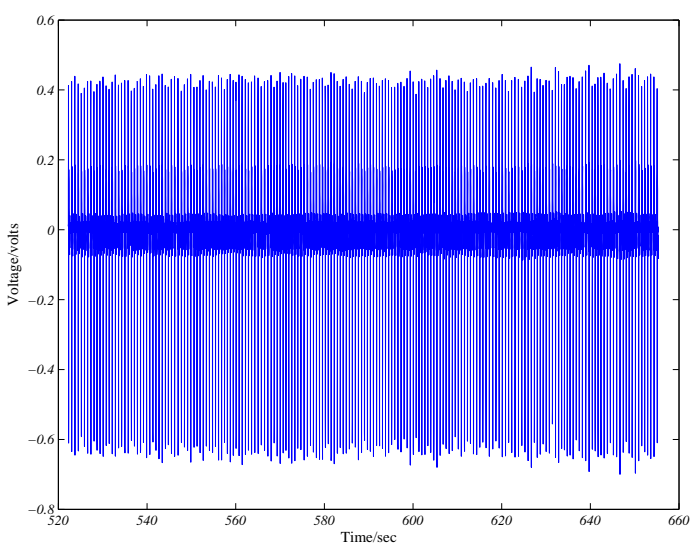

(c) Blue-high light

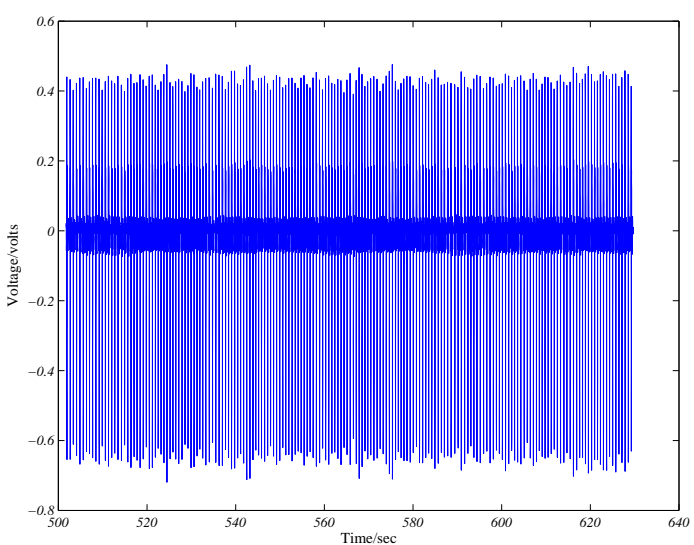

(b) Blue-low light

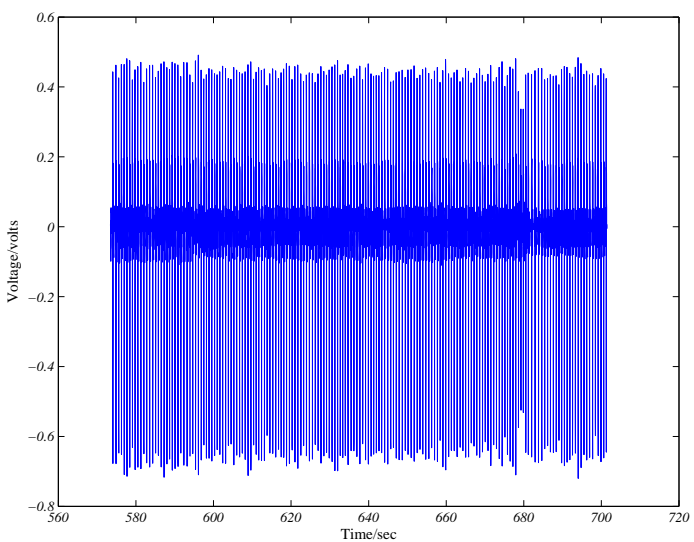

(d) Green-low light

Figure A.5: Participant 5 - ECG Recordings for participant 5 for all colour lights 


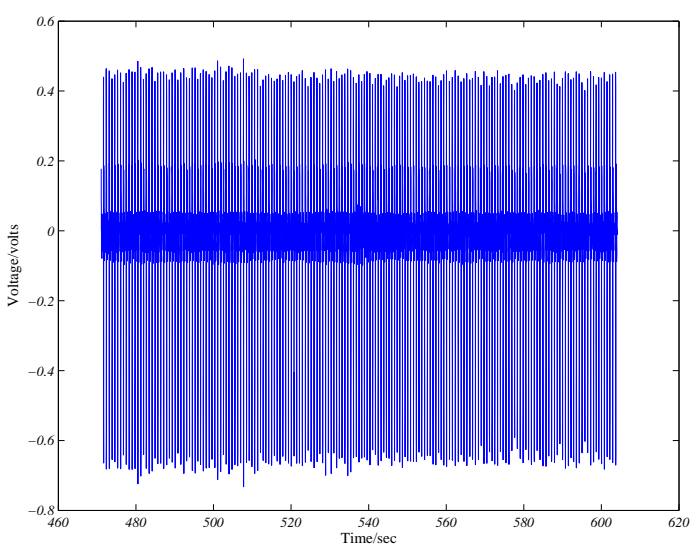

(e) Green-high light

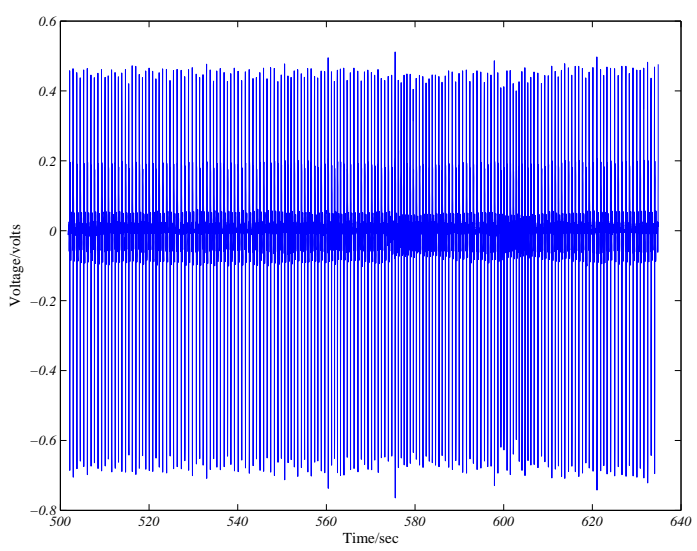

(g) Red-hig light

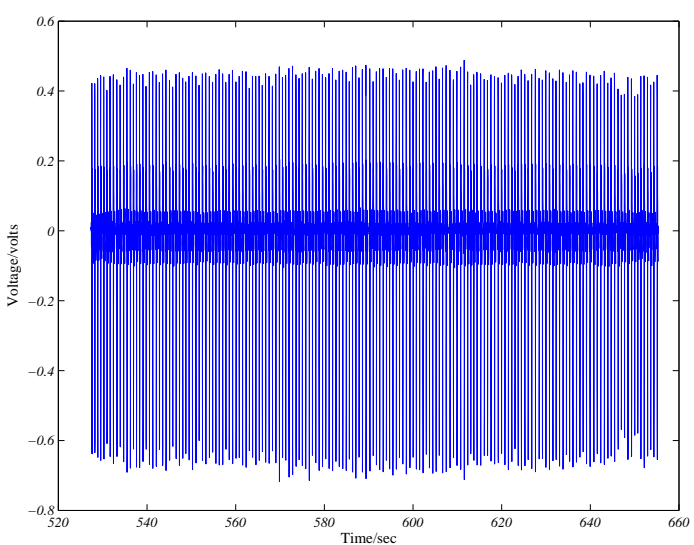

(f) Red-low light

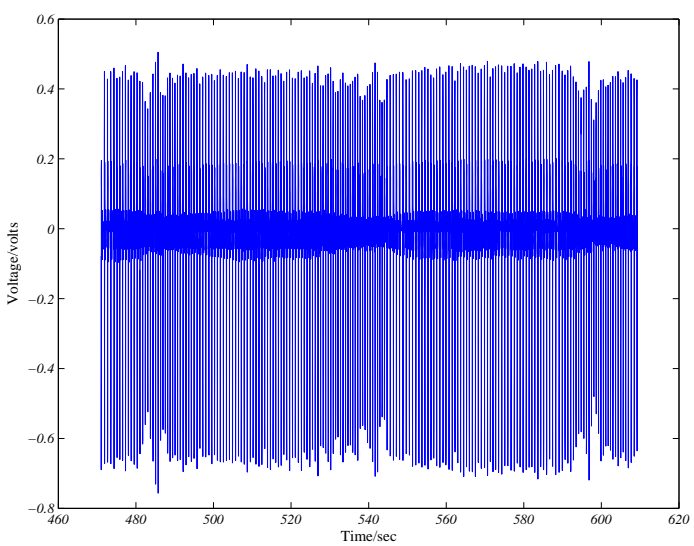

(h) Natural light

Figure A.5: Participant 5 - ECG Recordings for participant 5 for all colour lights 


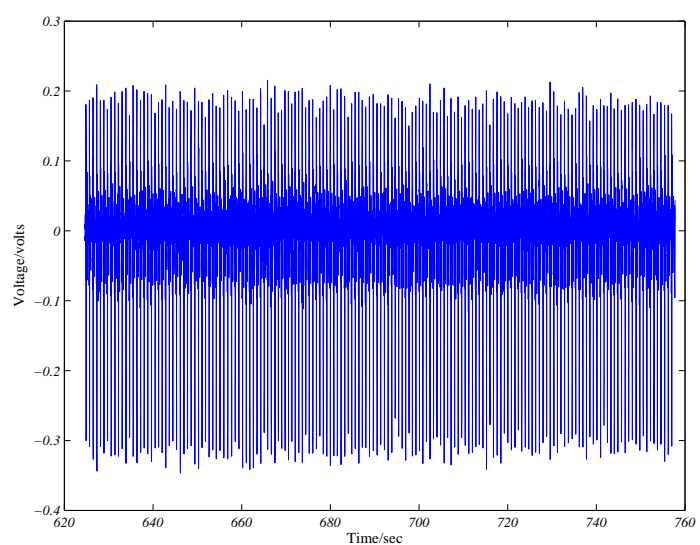

(a) White light

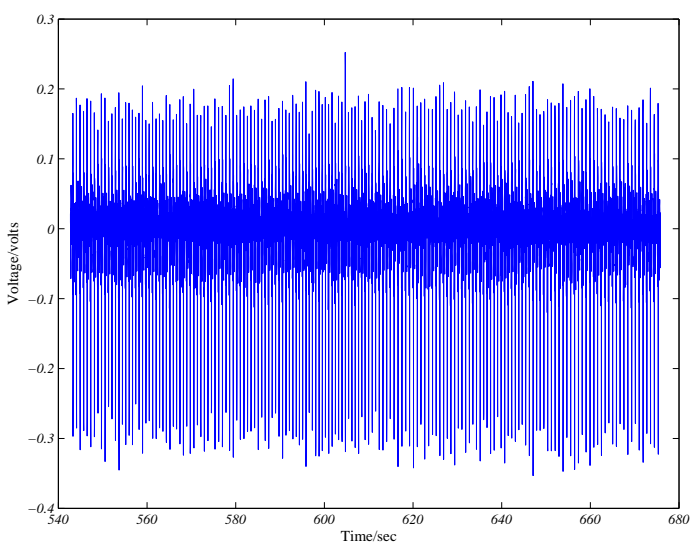

(c) Blue-high light

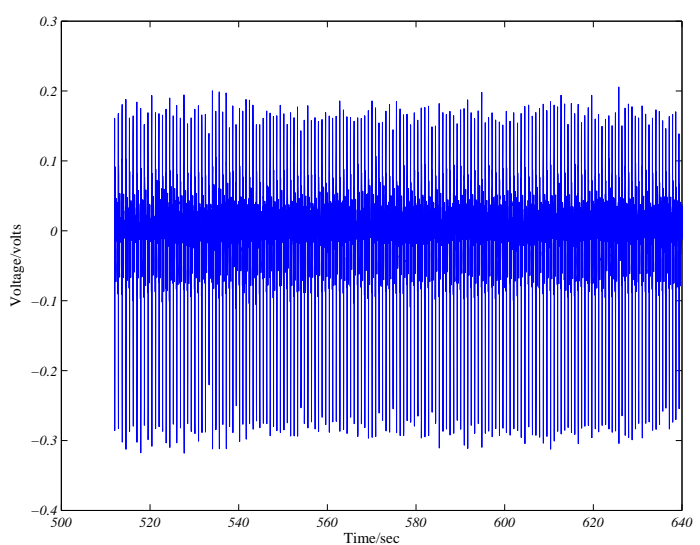

(b) Blue-low light

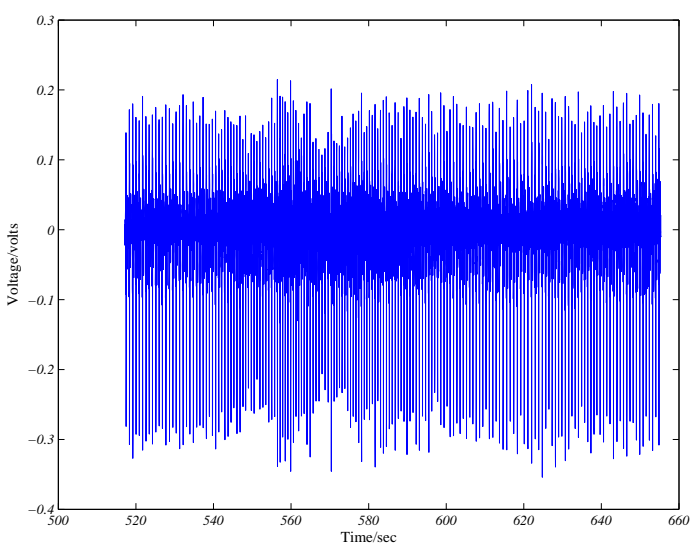

(d) Green-low light

Figure A.6: Participant 6 - ECG Recordings for participant 6 for all colour lights 


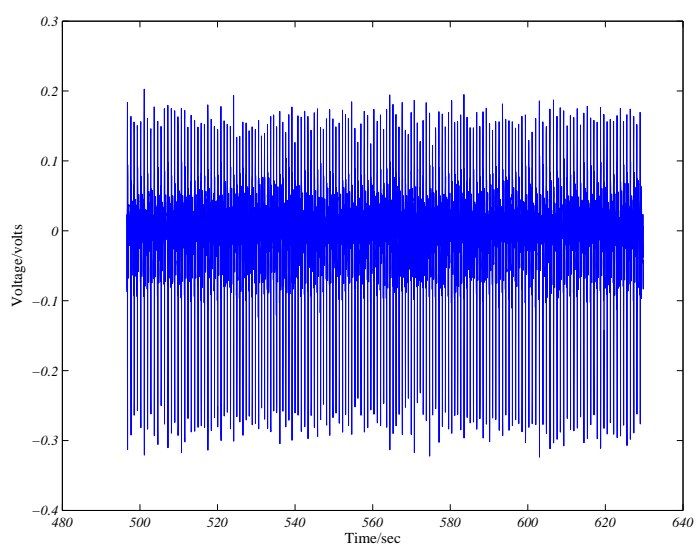

(e) Green-high light

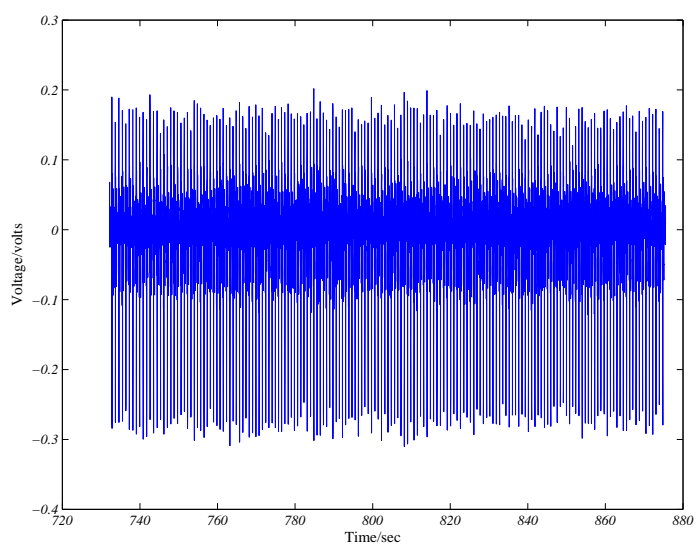

(g) Red-hig light

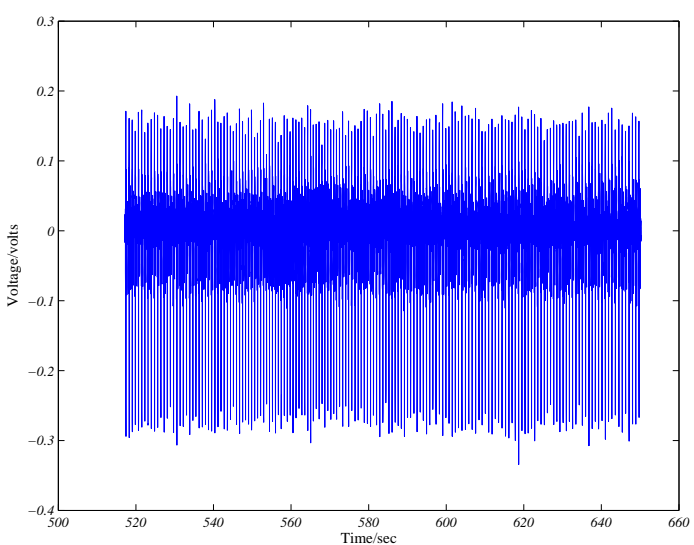

(f) Red-low light

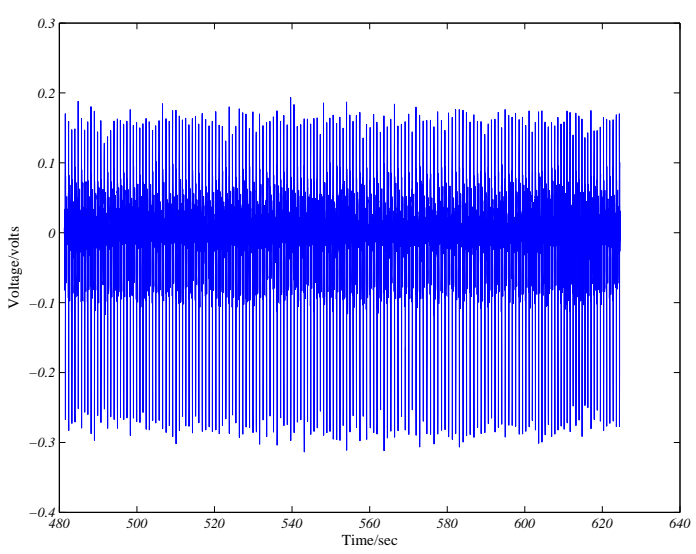

(h) Natural light

Figure A.6: Participant 6 - ECG Recordings for participant 6 for all colour lights 


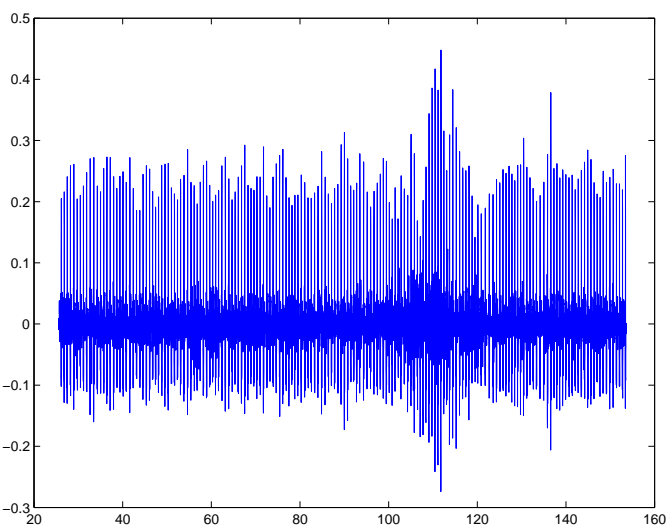

(a) White light

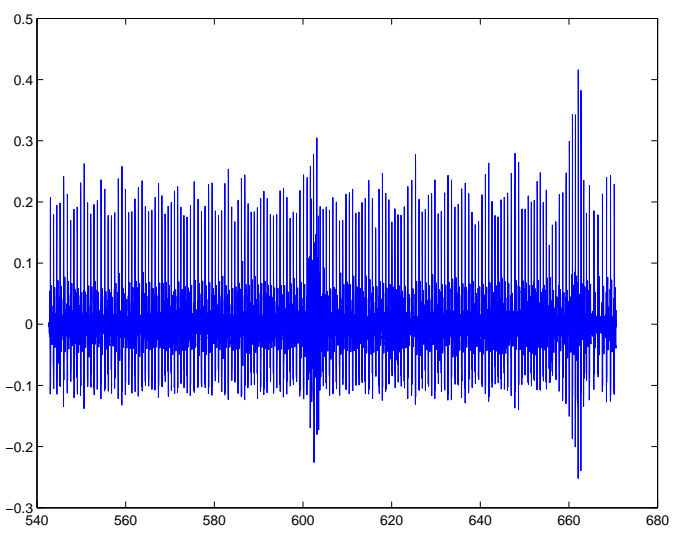

(c) Blue-high light

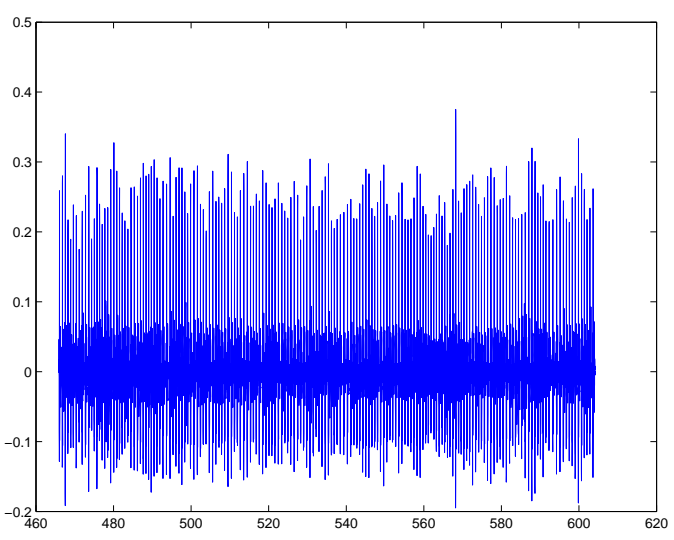

(b) Blue-low light

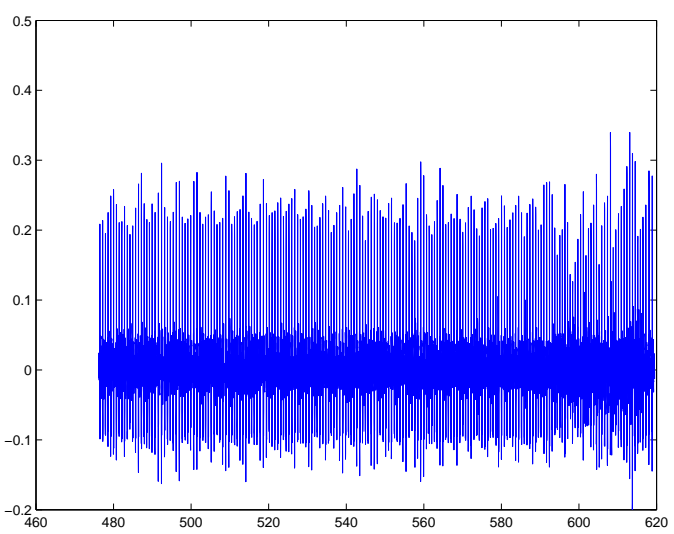

(d) Green-low light

Figure A.7: Participant 7 - ECG Recordings for participant 7 for all colour lights 


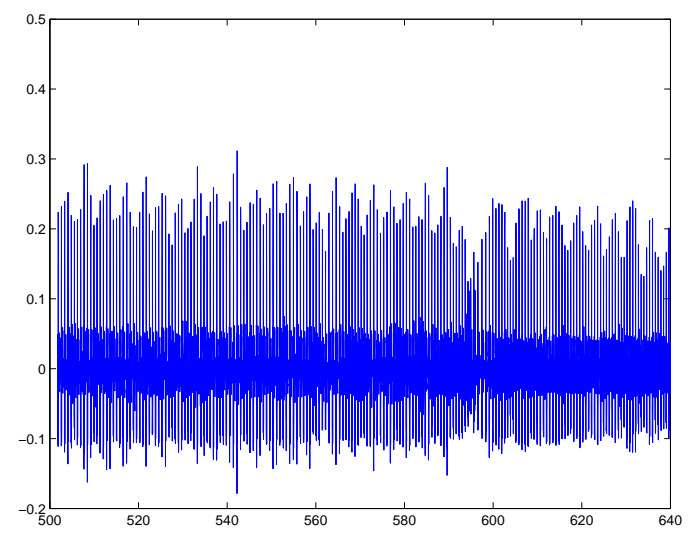

(e) Green-high light

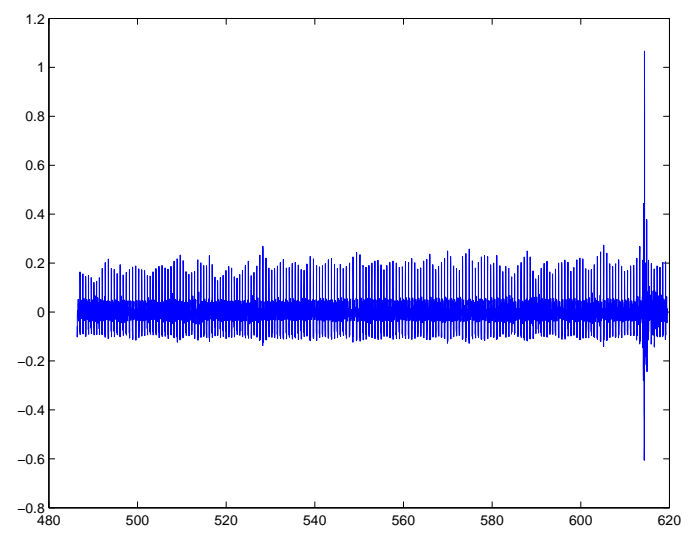

(g) Red-hig light

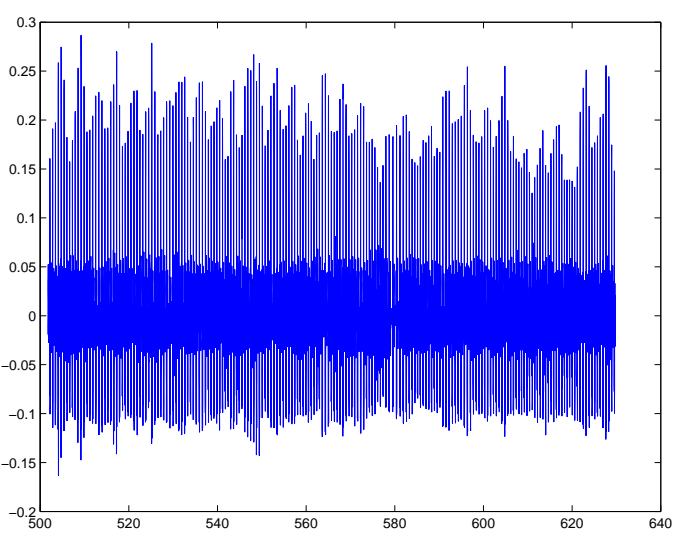

(f) Red-low light

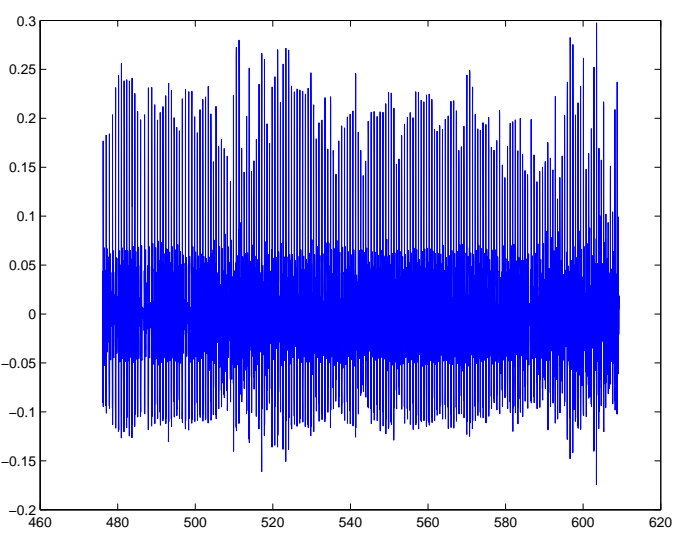

(h) Natural light

Figure A.7: Participant 7 - ECG Recordings for participant 7 for all colour lights 


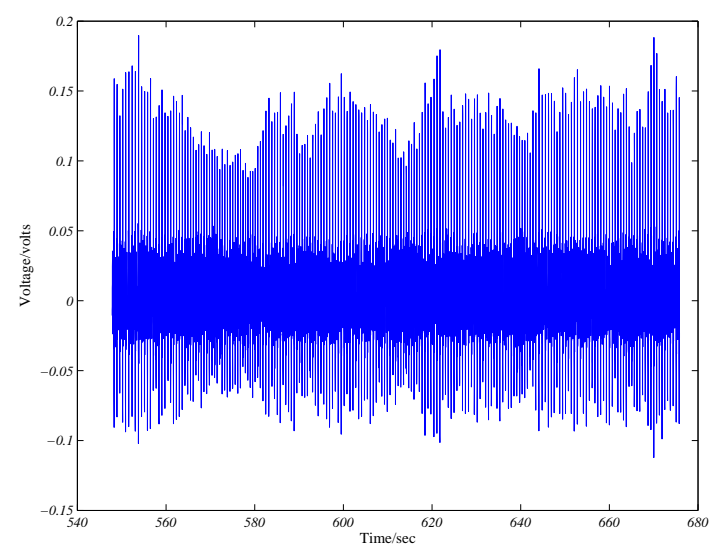

(a) White light

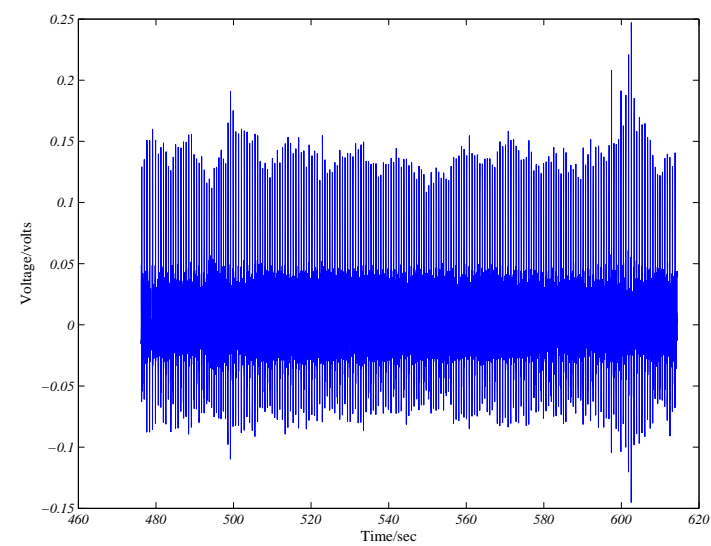

(c) Blue-high light

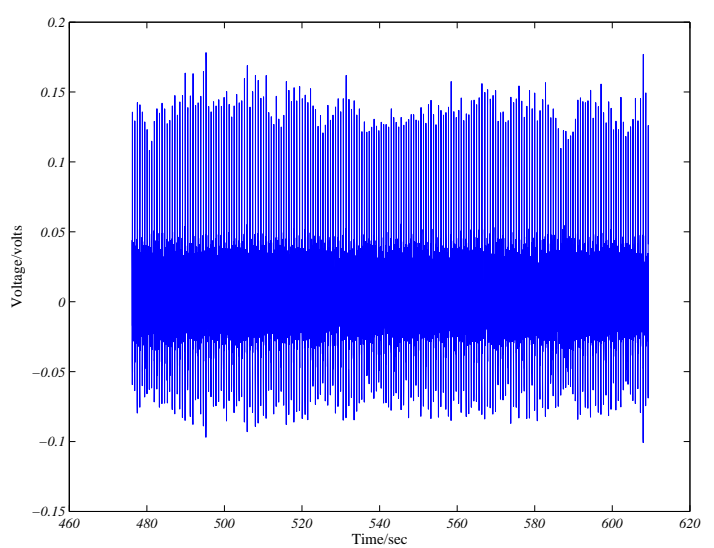

(b) Blue-low light

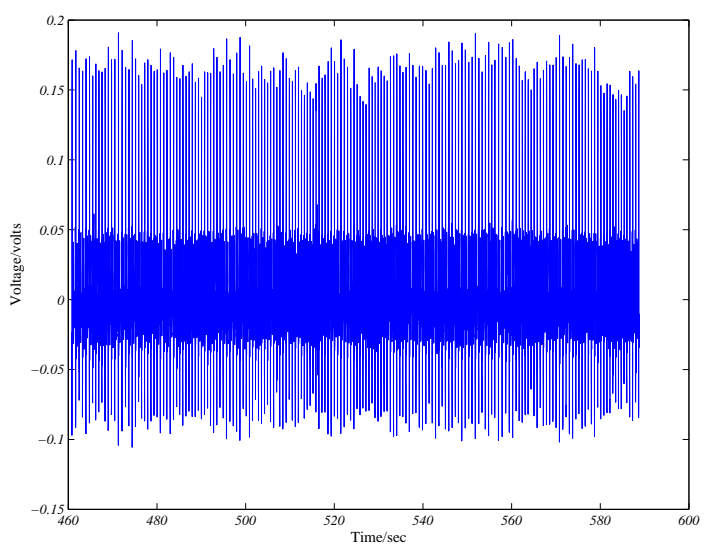

(d) Green-low light

Figure A.8: Participant 8 - ECG Recordings for participant 8 for all colour lights 


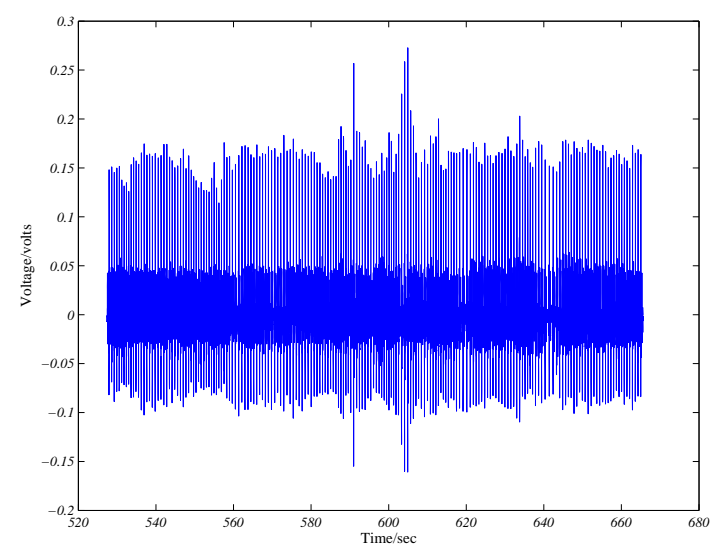

(e) Green-high light

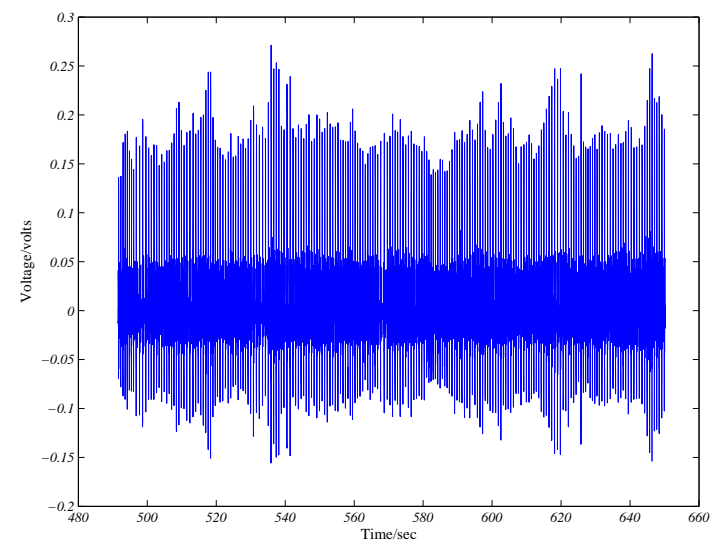

(g) Red-hig light

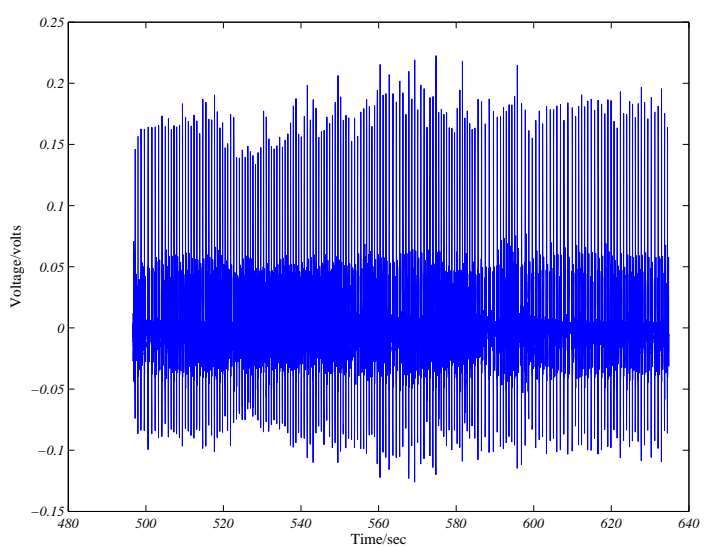

(f) Red-low light

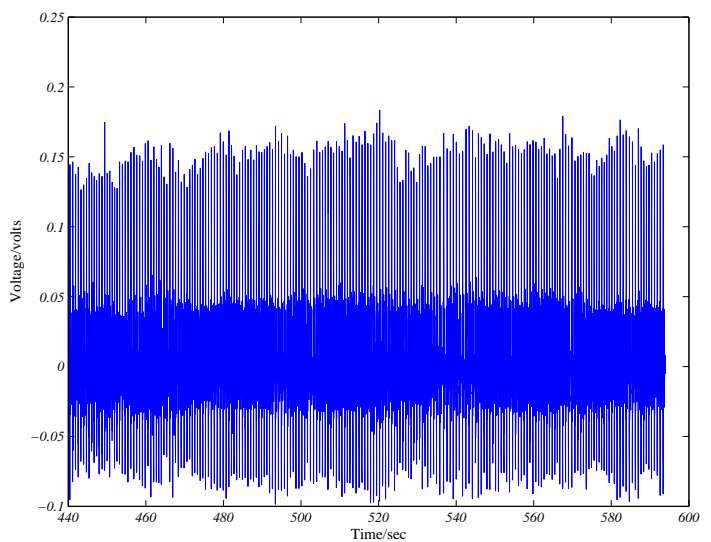

(h) Natural light

Figure A.8: Participant 8 - ECG Recordings for participant 8 for all colour lights 


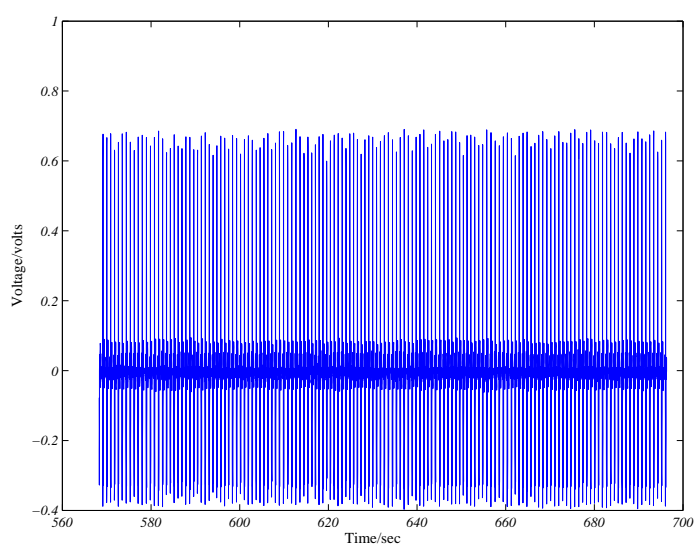

(a) White light

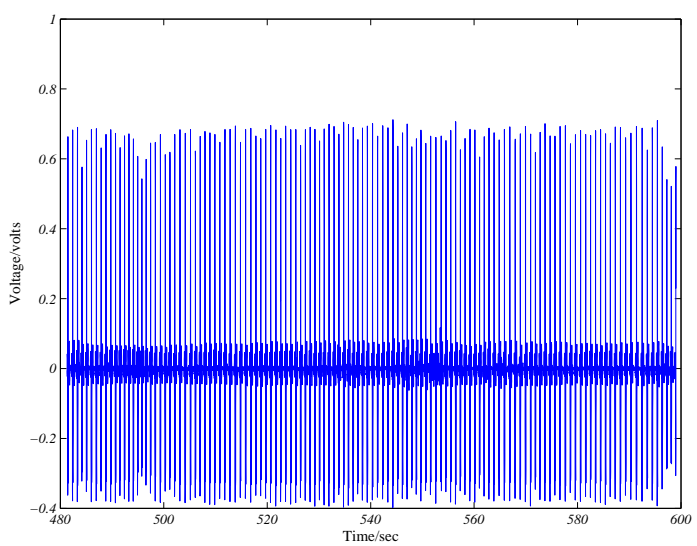

(c) Blue-high light

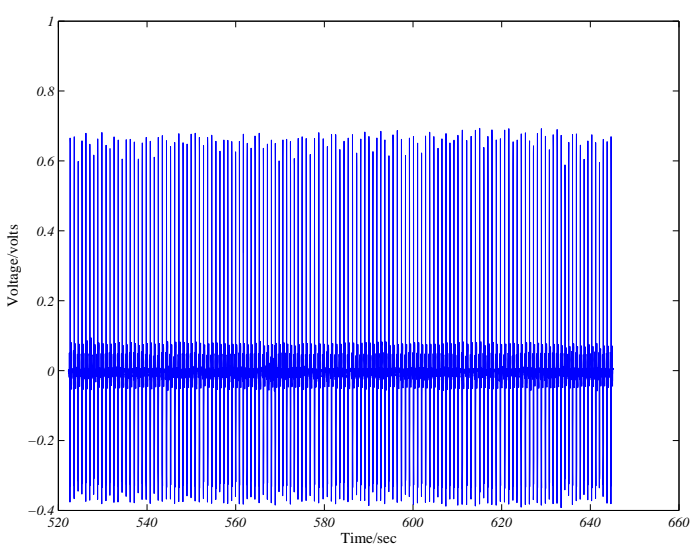

(b) Blue-low light

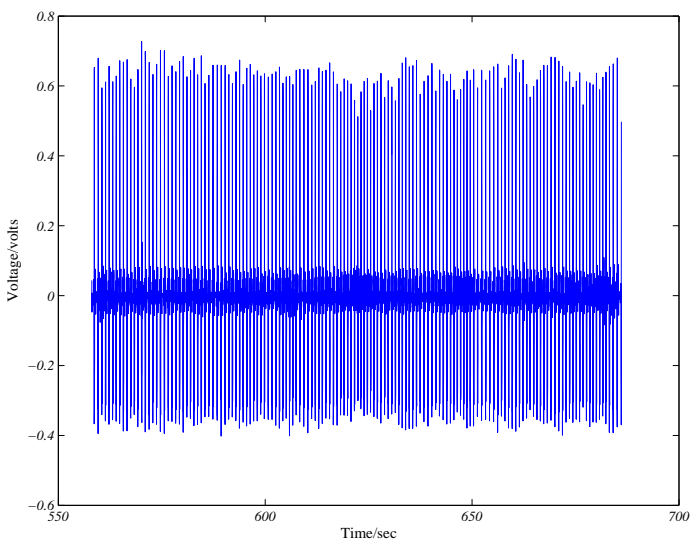

(d) Green-low light

Figure A.9: Participant 9 - ECG Recordings for participant 9 for all colour lights 


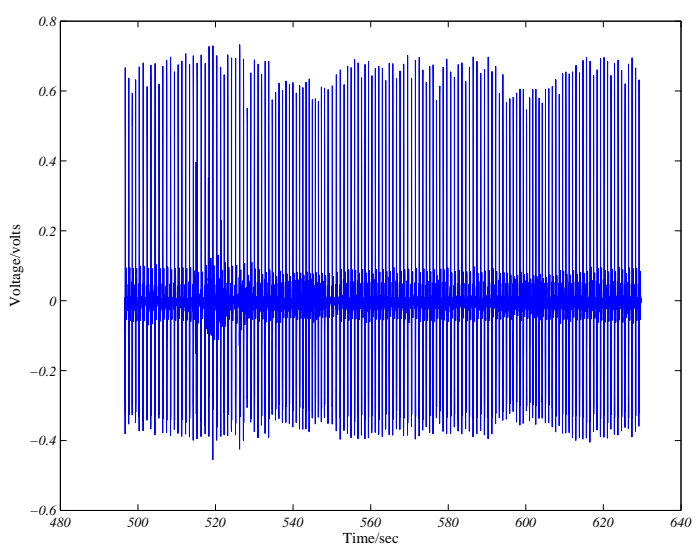

(e) Green-high light

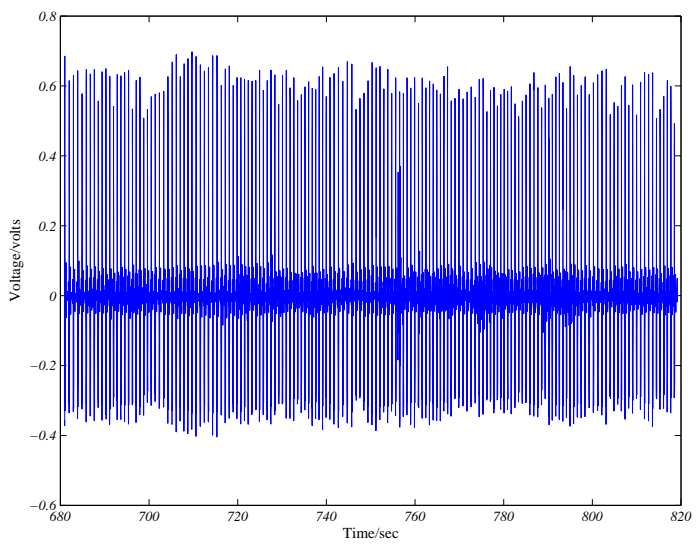

(g) Red-hig light

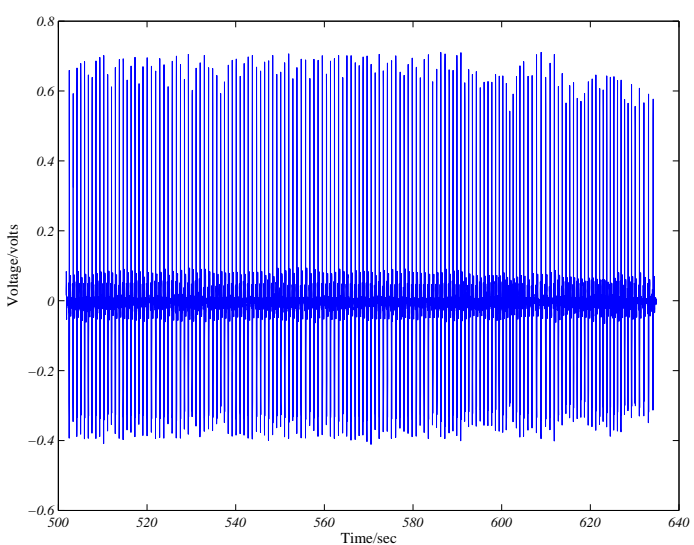

(f) Red-low light

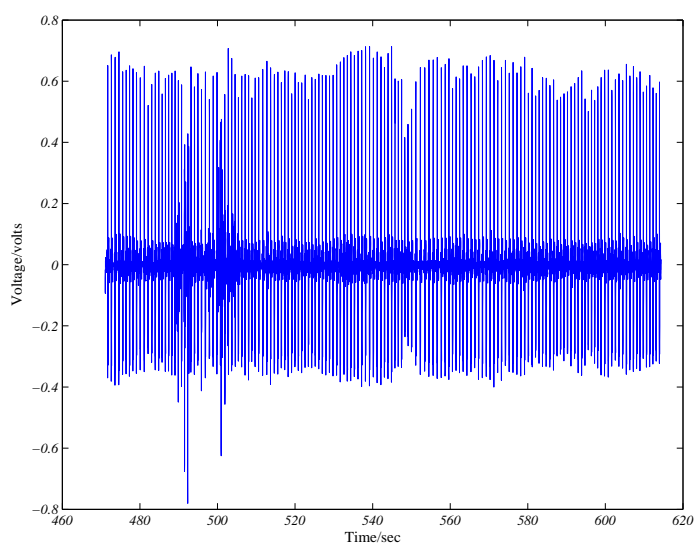

(h) Natural light

Figure A.9: Participant 9 - ECG Recordings for participant 9 for all colour lights 


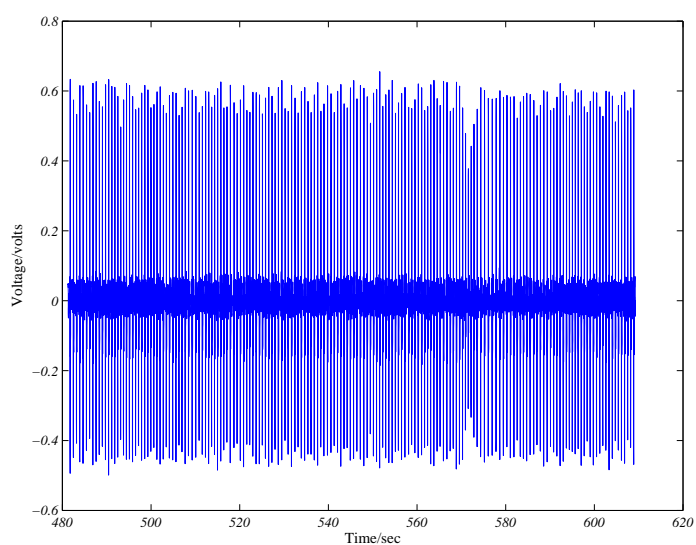

(a) White light

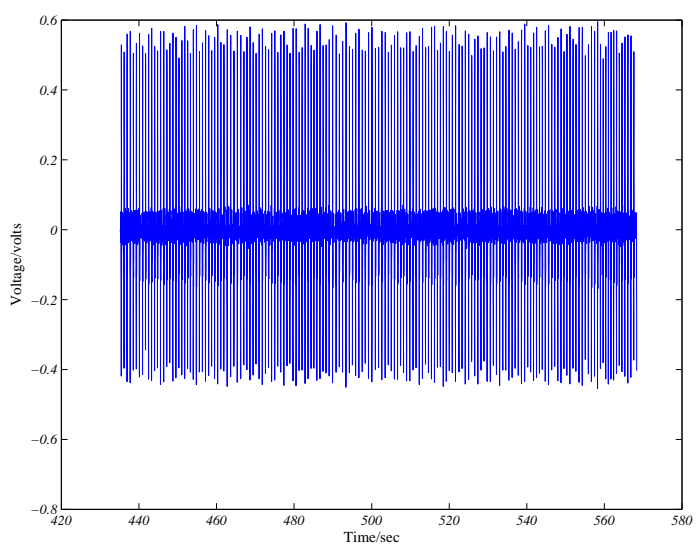

(c) Blue-high light

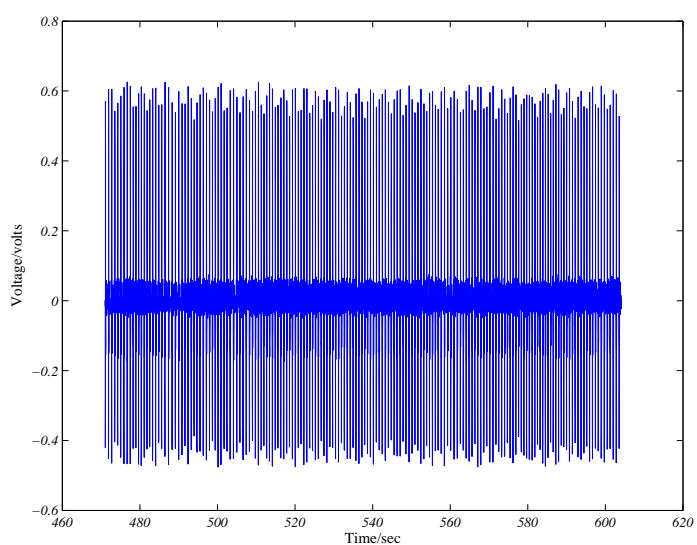

(b) Blue-low light

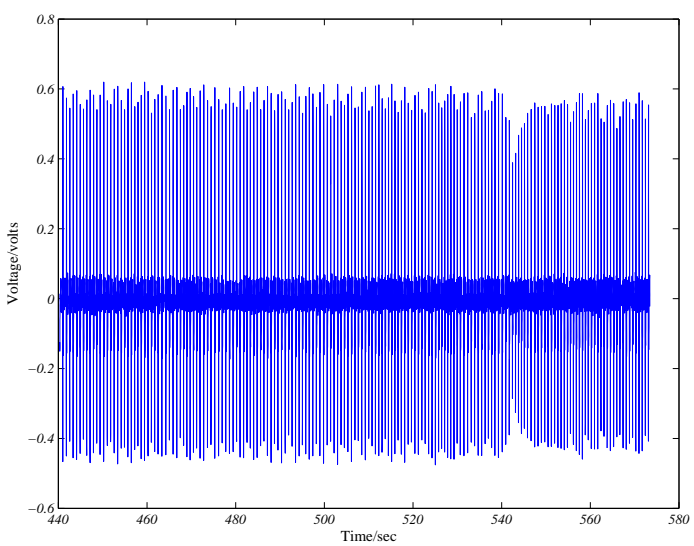

(d) Green-low light

Figure A.10: Participant 10 - ECG Recordings for participant 10 for all colour lights 


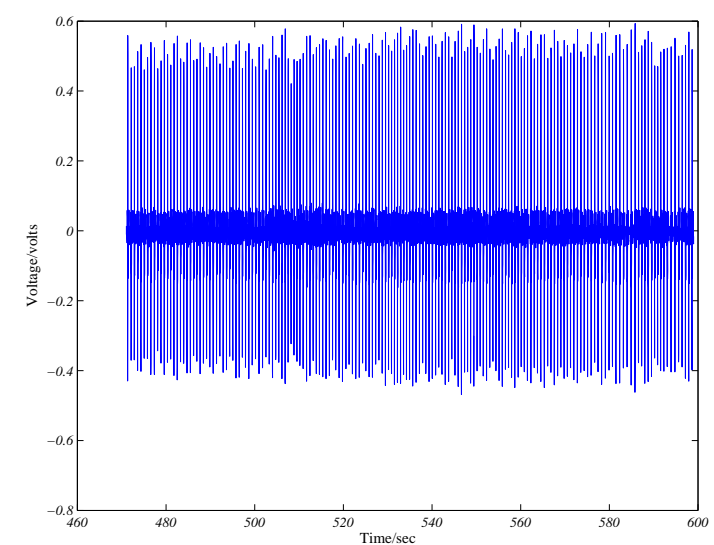

(e) Green-high light

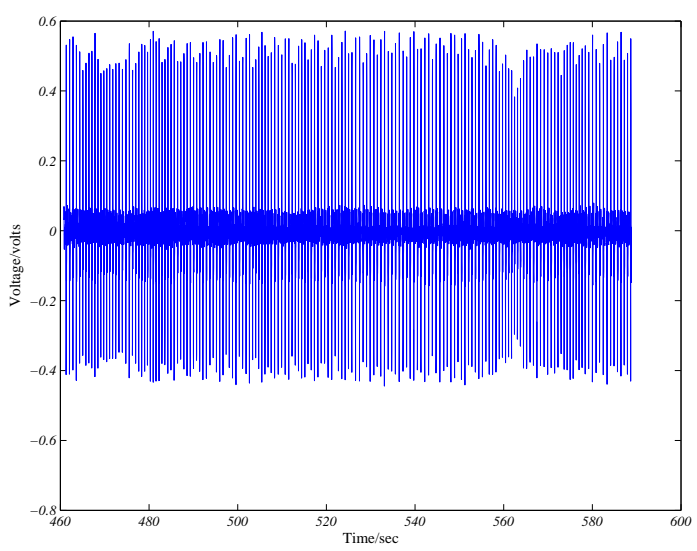

(g) Red-hig light

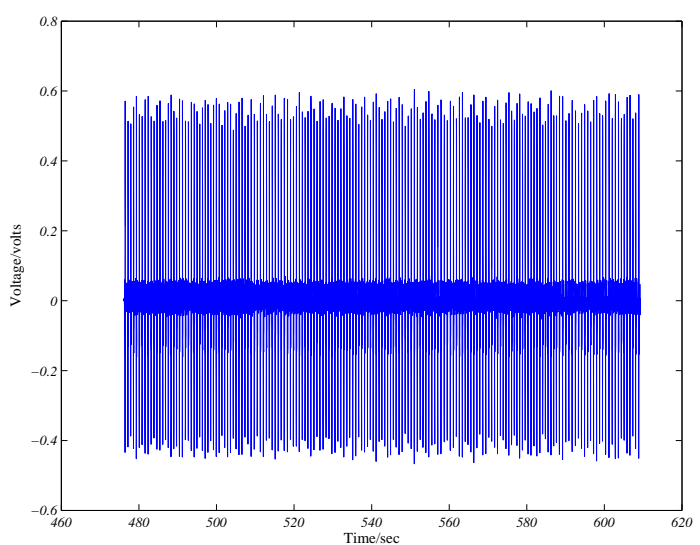

(f) Red-low light

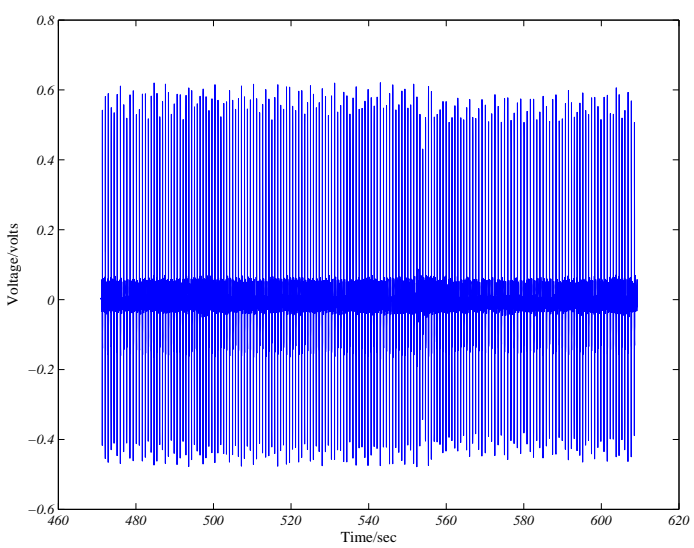

(h) Natural light

Figure A.10: Participant 10 - ECG Recordings for participant 10 for all colour lights 


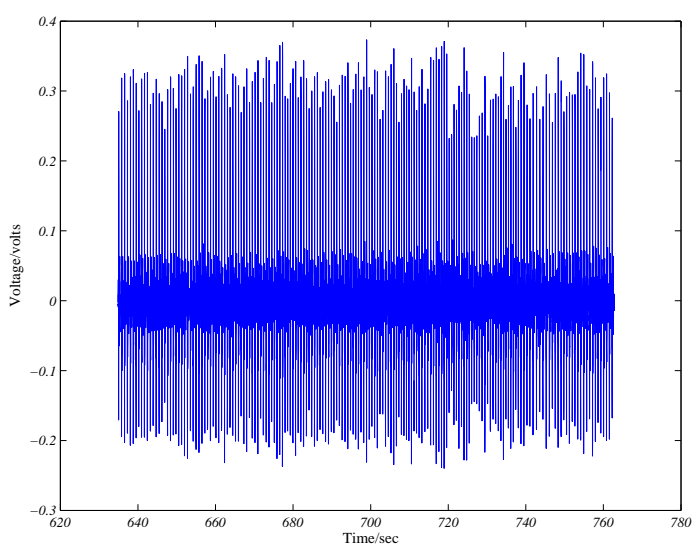

(a) White light

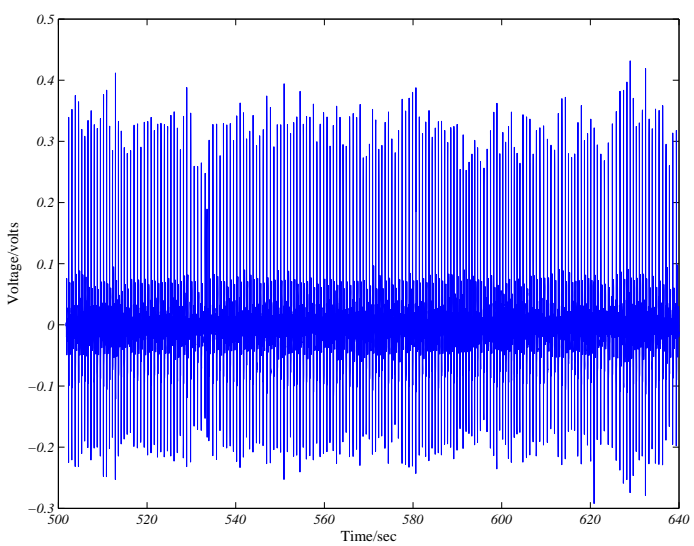

(c) Blue-high light

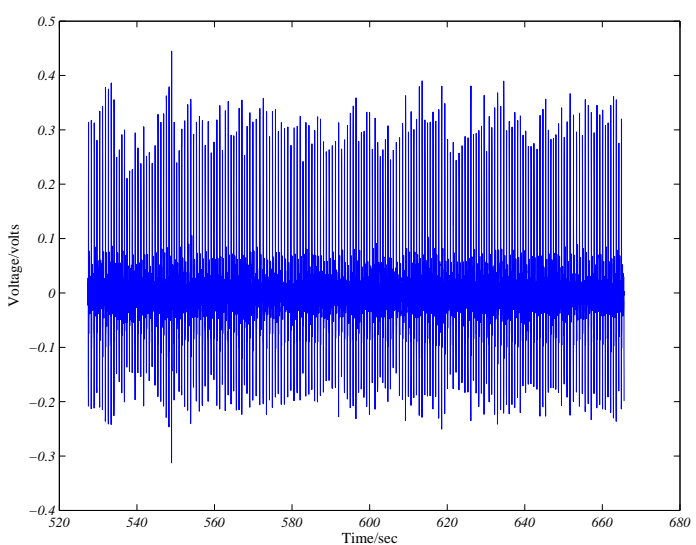

(b) Blue-low light

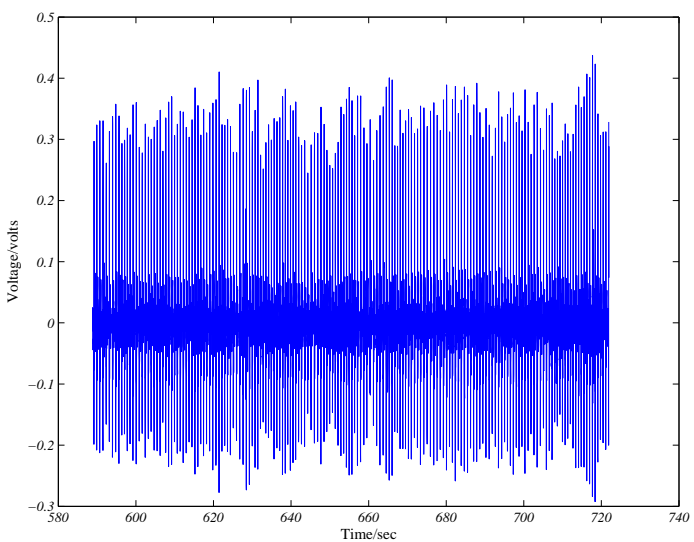

(d) Green-low light

Figure A.11: Participant 11 - ECG Recordings for participant 11 for all colour lights 


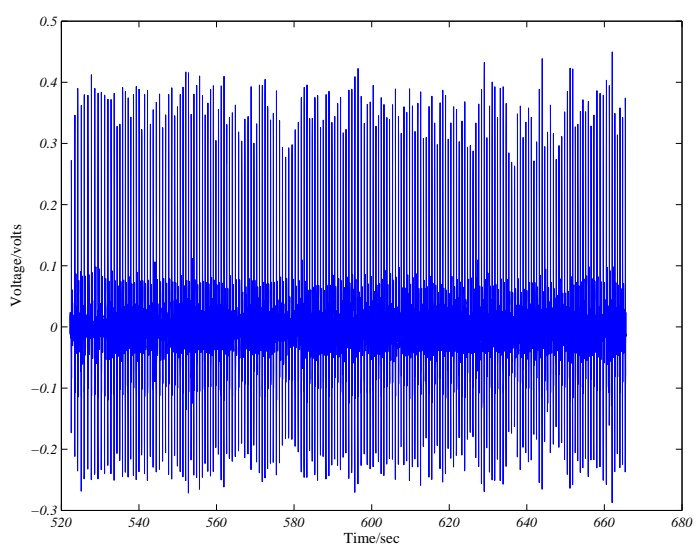

(e) Green-high light

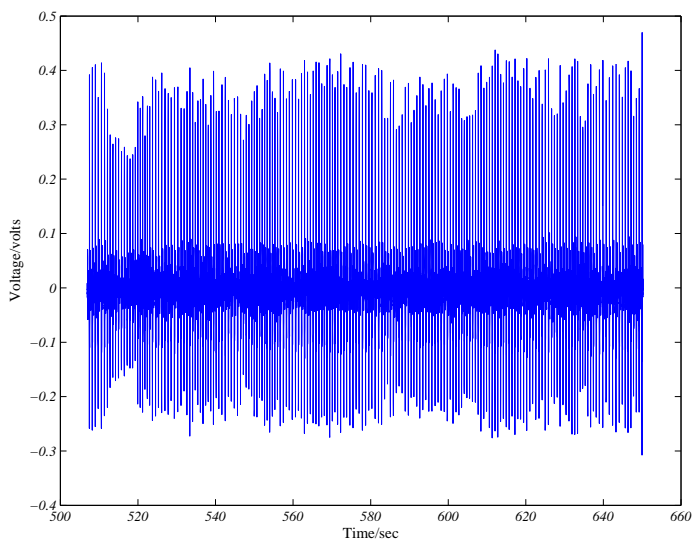

(g) Red-hig light

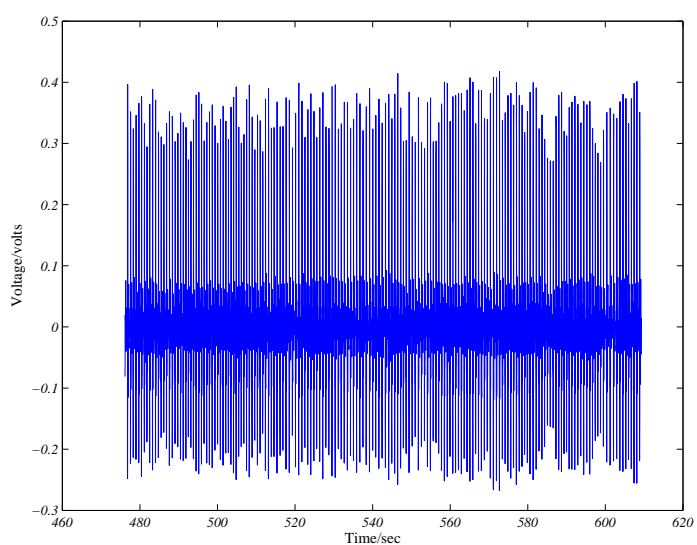

(f) Red-low light

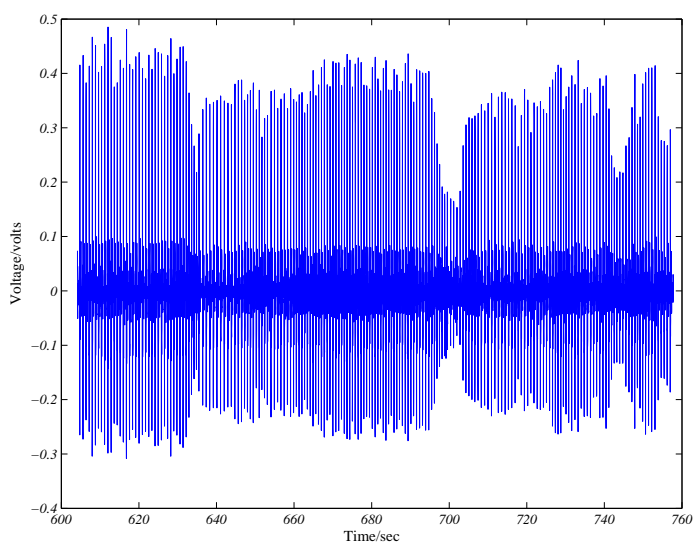

(h) Natural light

Figure A.11: Participant 11 - ECG Recordings for participant 11 for all colour lights 


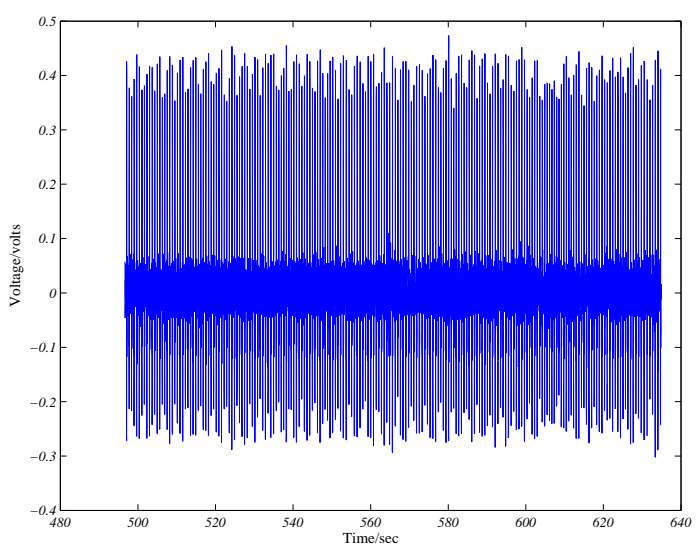

(a) White light

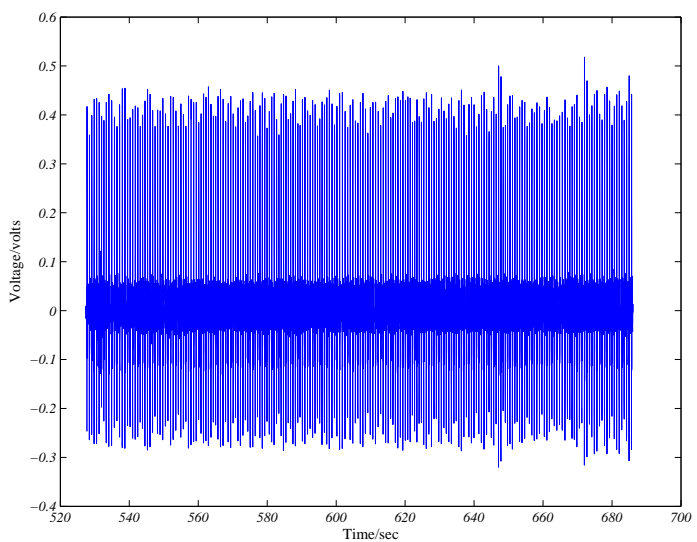

(c) Blue-high light

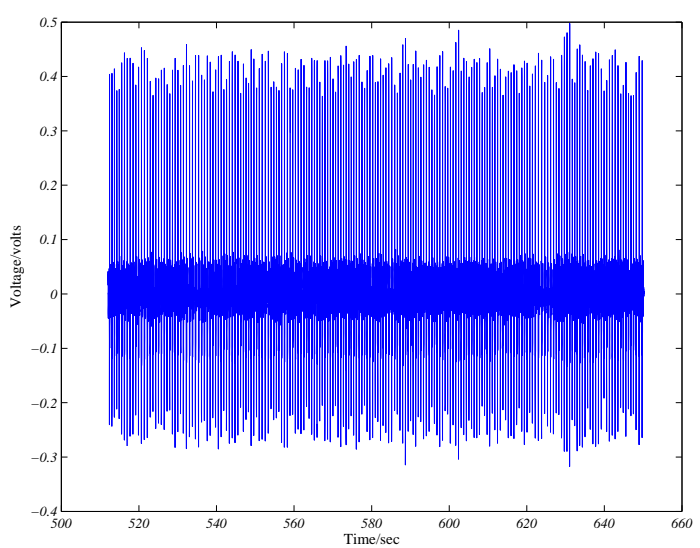

(b) Blue-low light

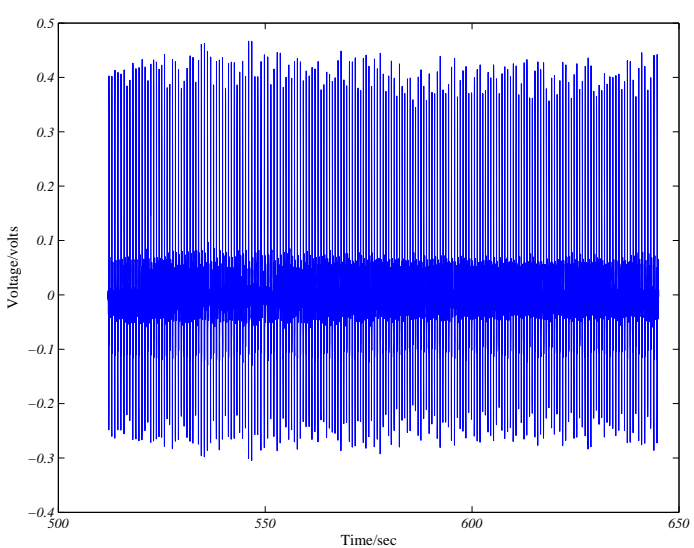

(d) Green-low light

Figure A.12: Participant 12 - ECG Recordings for participant 12 for all colour lights 


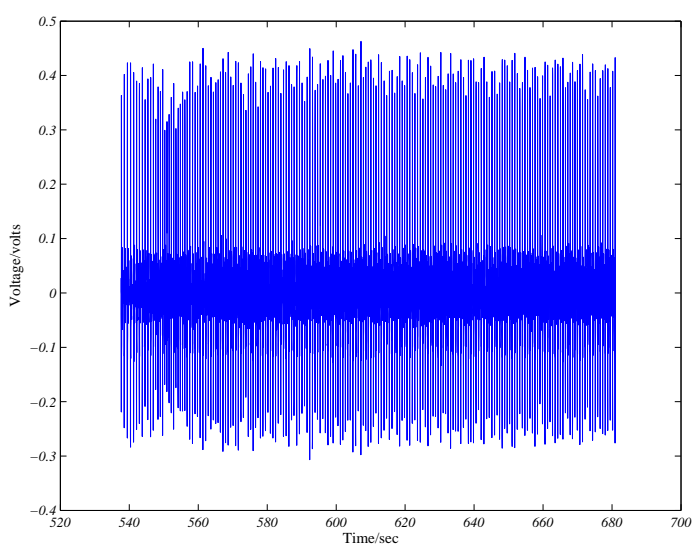

(e) Green-high light

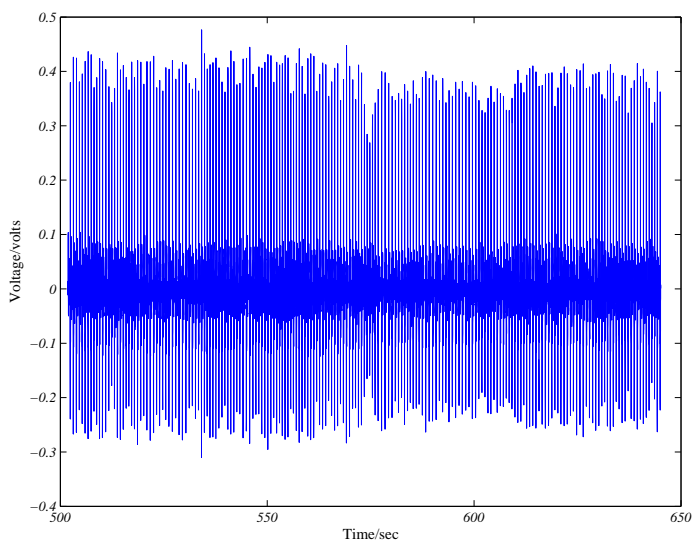

(g) Red-hig light

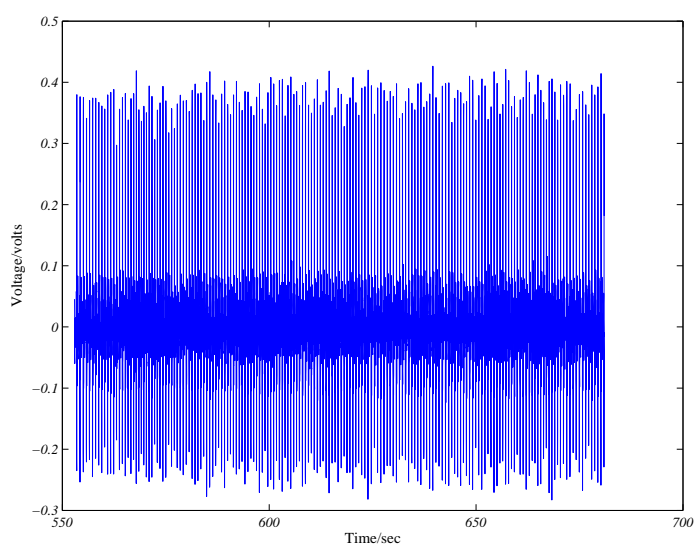

(f) Red-low light

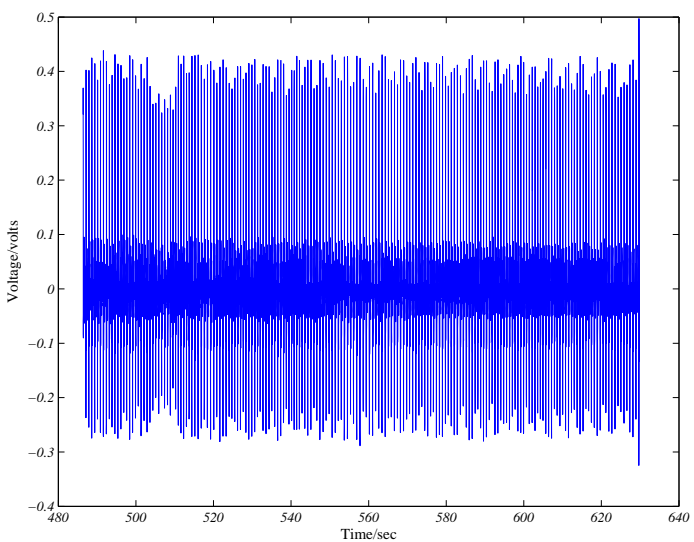

(h) Natural light

Figure A.12: Participant 12 - ECG Recordings for participant 12 for all colour lights 


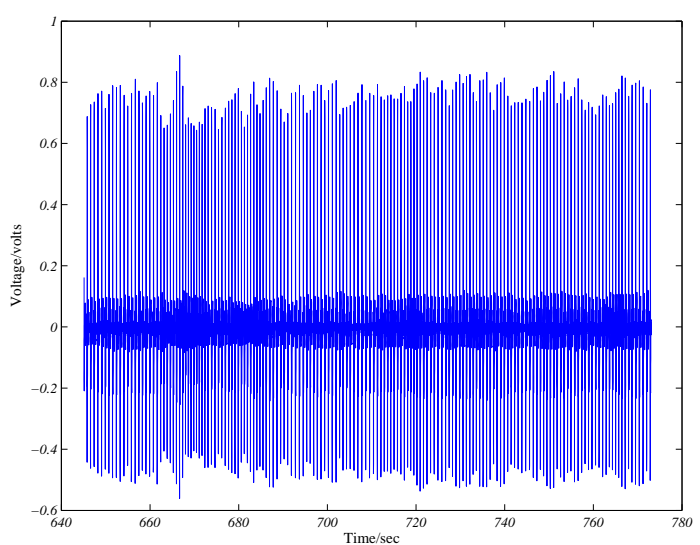

(a) White light

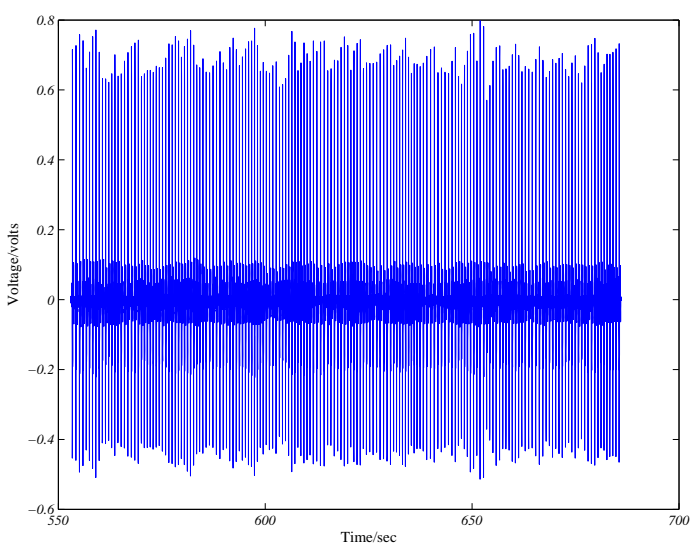

(c) Blue-high light

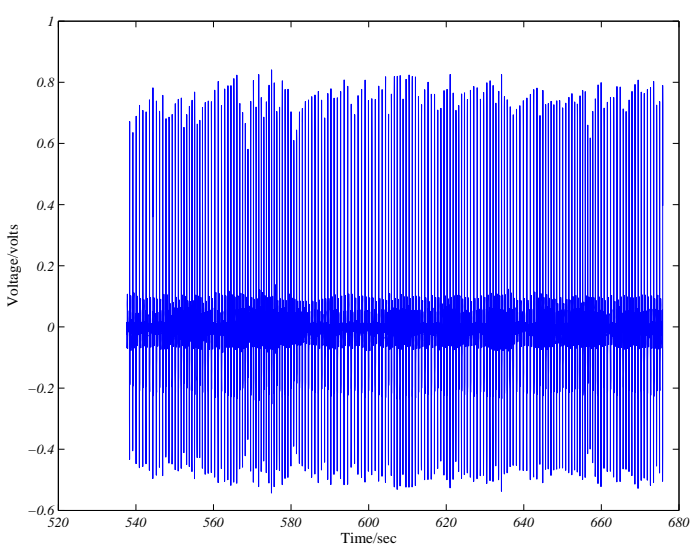

(b) Blue-low light

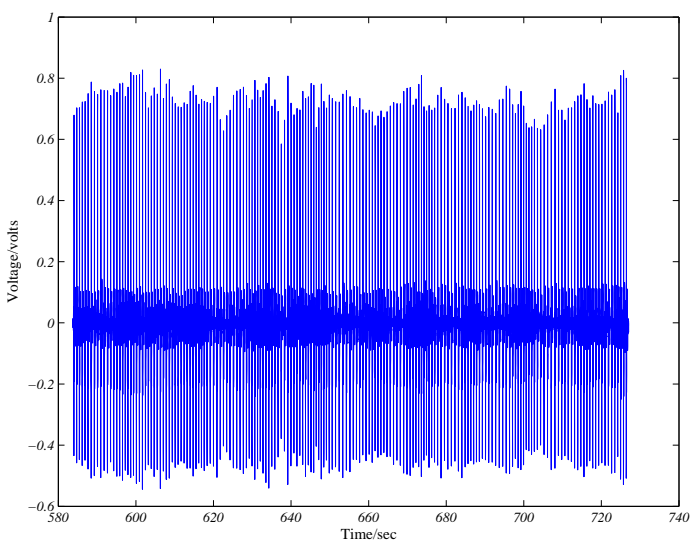

(d) Green-low light

Figure A.13: Participant 13 - ECG Recordings for participant 13 for all colour lights 


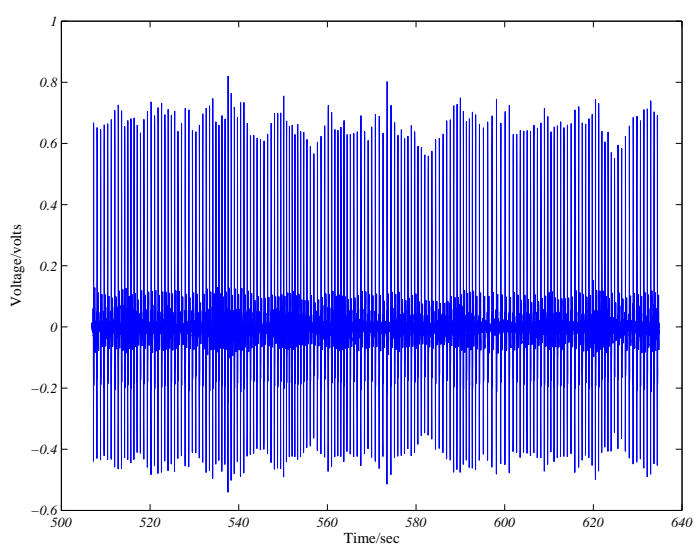

(e) Green-high light

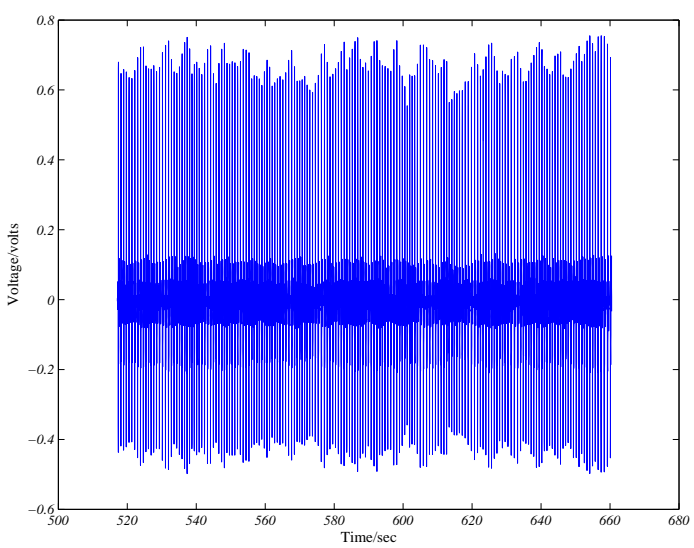

(g) Red-hig light

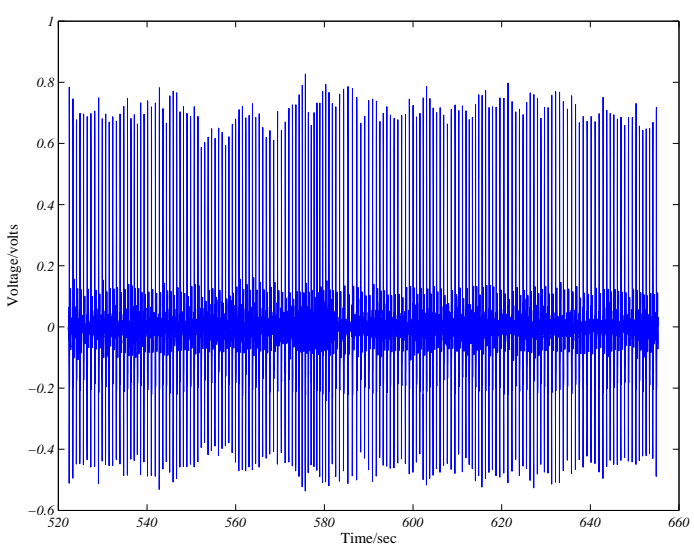

(f) Red-low light

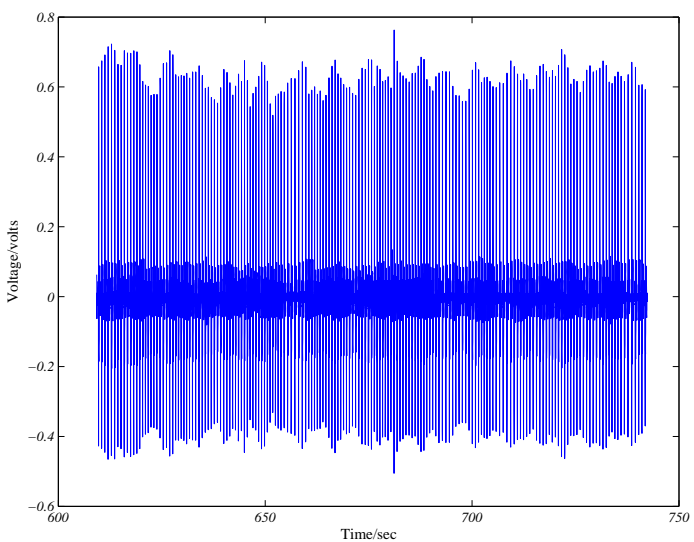

(h) Natural light

Figure A.13: Participant 13 - ECG Recordings for participant 13 for all colour lights 


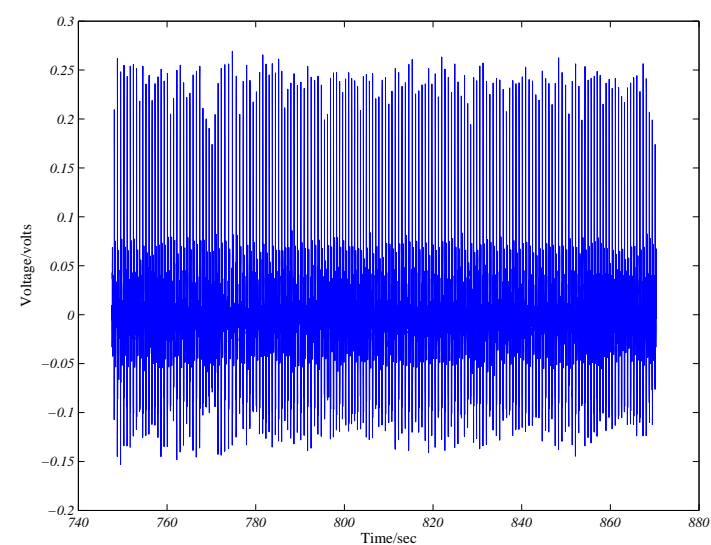

(a) White light

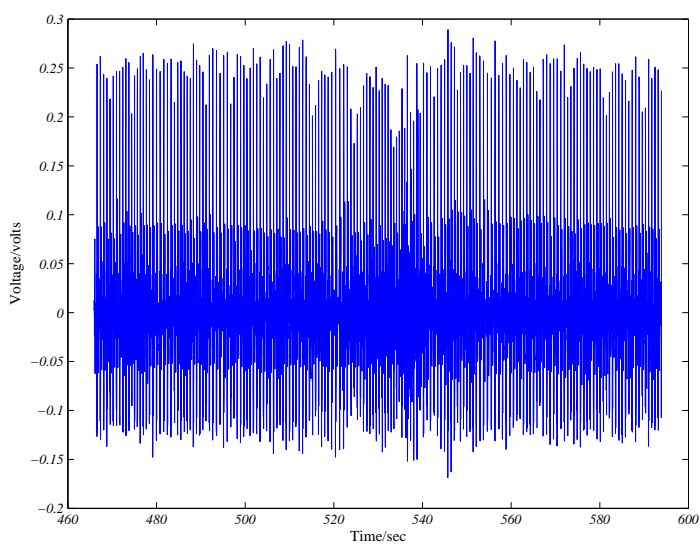

(c) Blue-high light

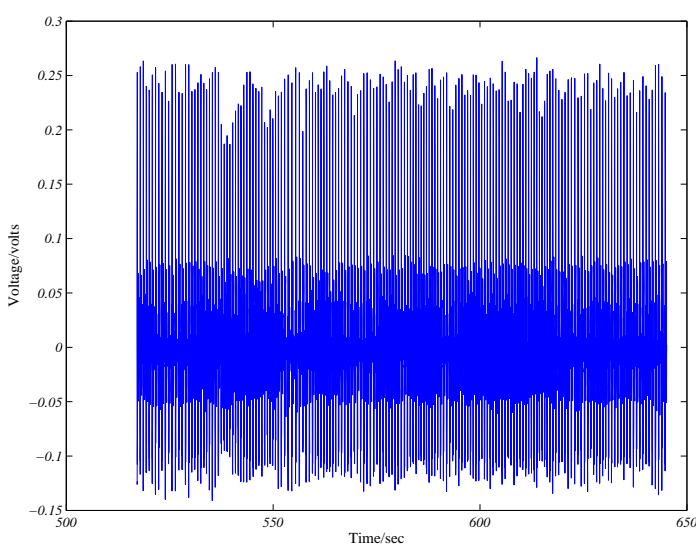

(b) Blue-low light

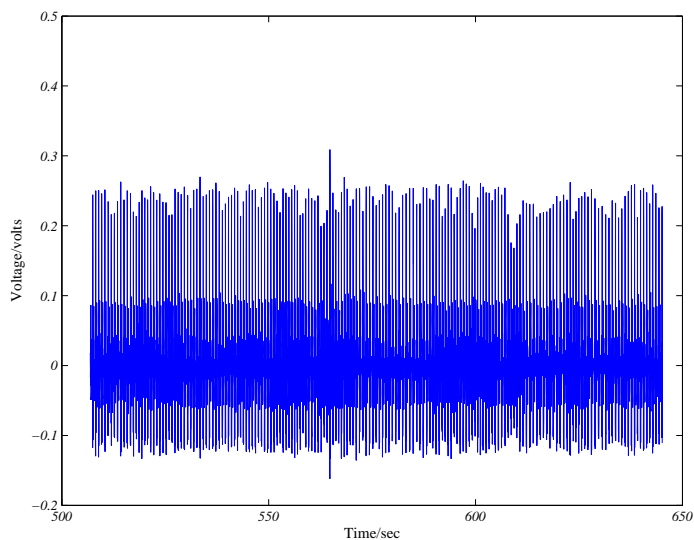

(d) Green-low light

Figure A.14: Participant 14 - ECG Recordings for participant 14 for all colour lights 


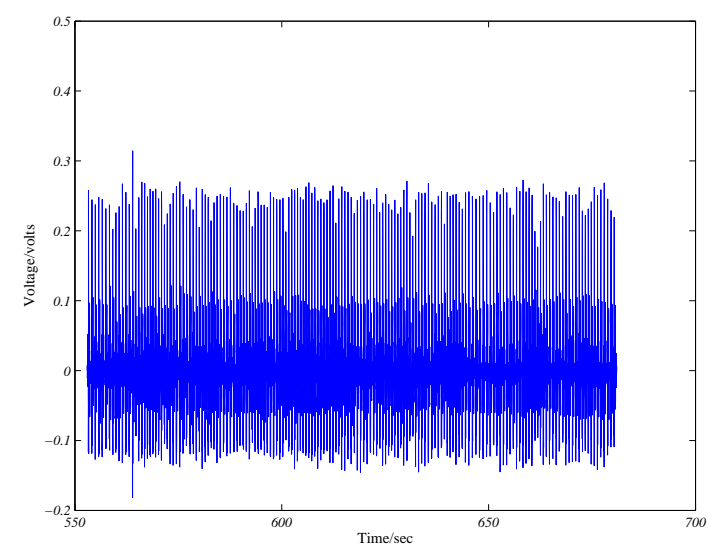

(e) Green-high light

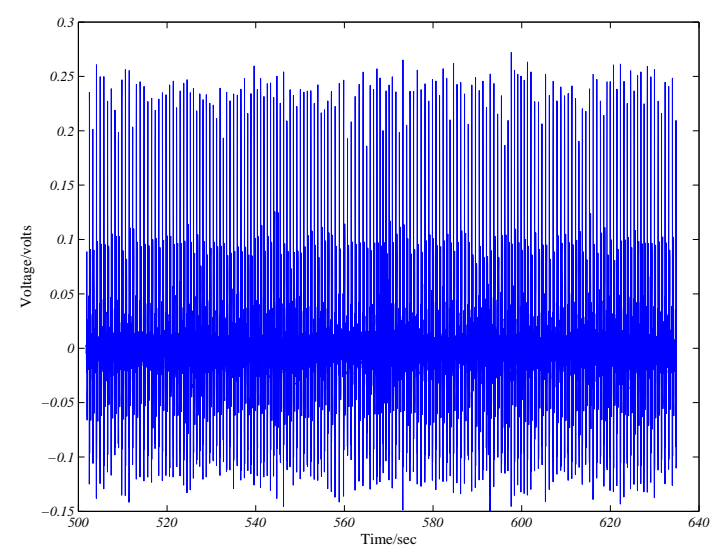

(g) Red-hig light

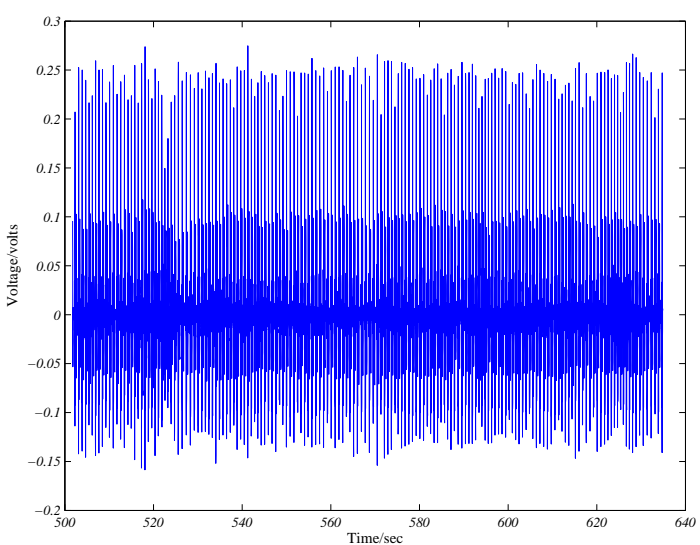

(f) Red-low light

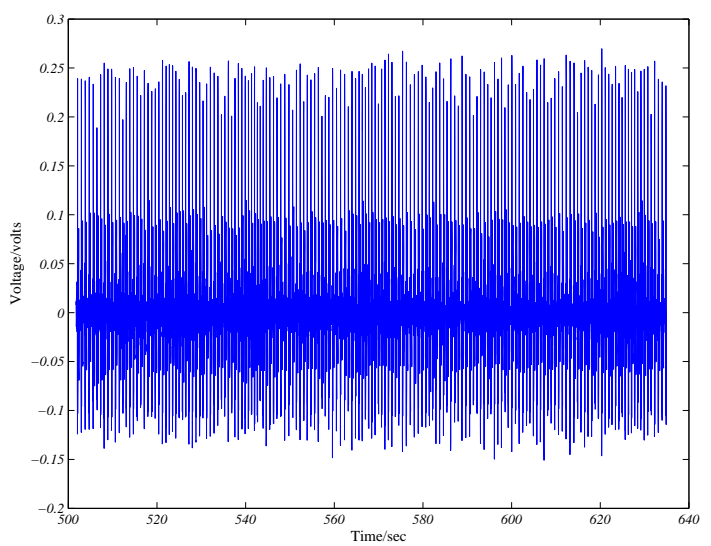

(h) Natural light

Figure A.14: Participant 14 - ECG Recordings for participant 14 for all colour lights 


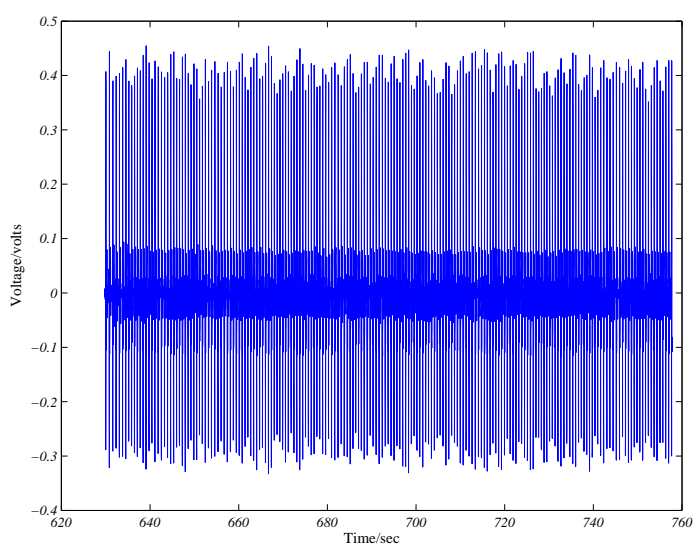

(a) White light

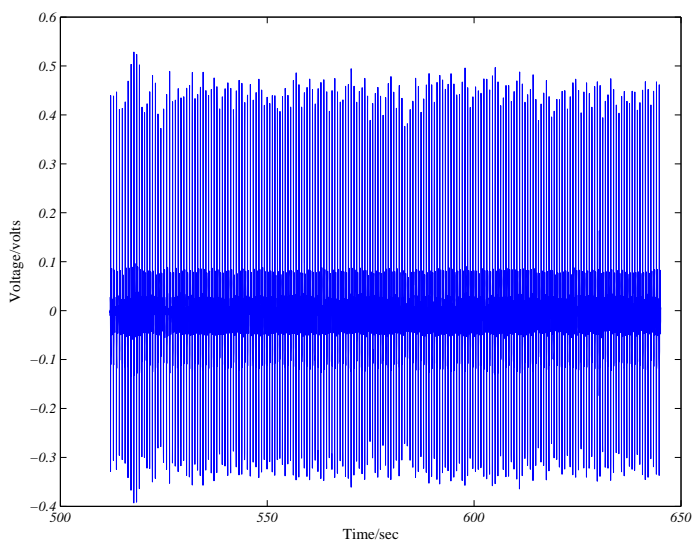

(c) Blue-high light

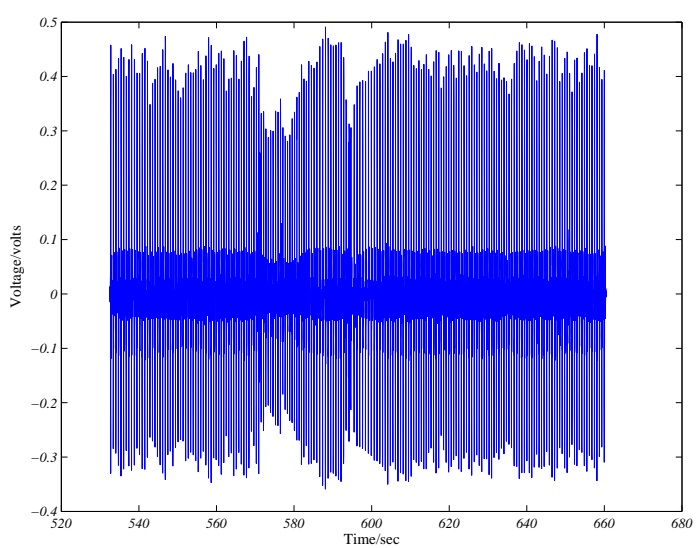

(b) Blue-low light

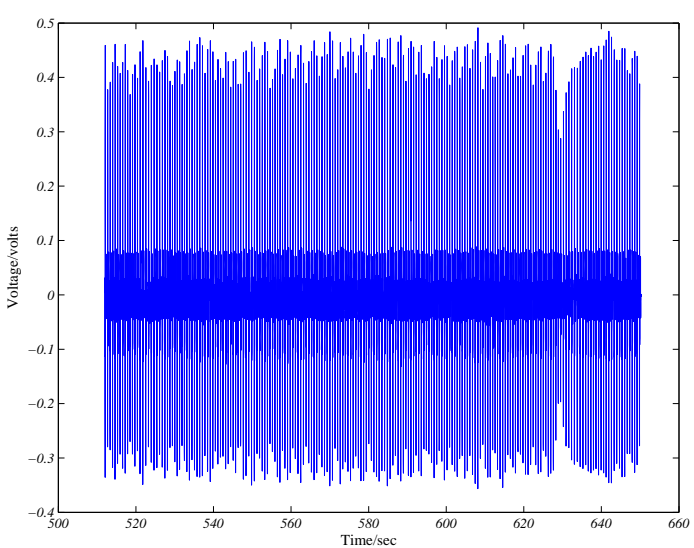

(d) Green-low light

Figure A.15: Participant 15 - ECG Recordings for participant 15 for all colour lights 


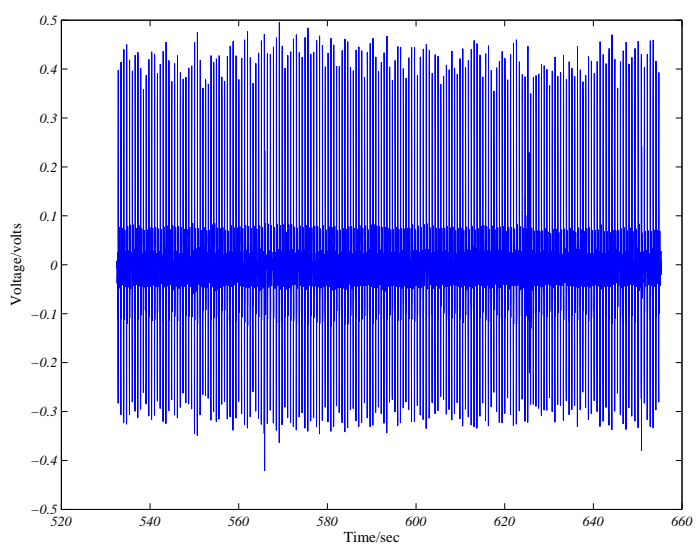

(e) Green-high light

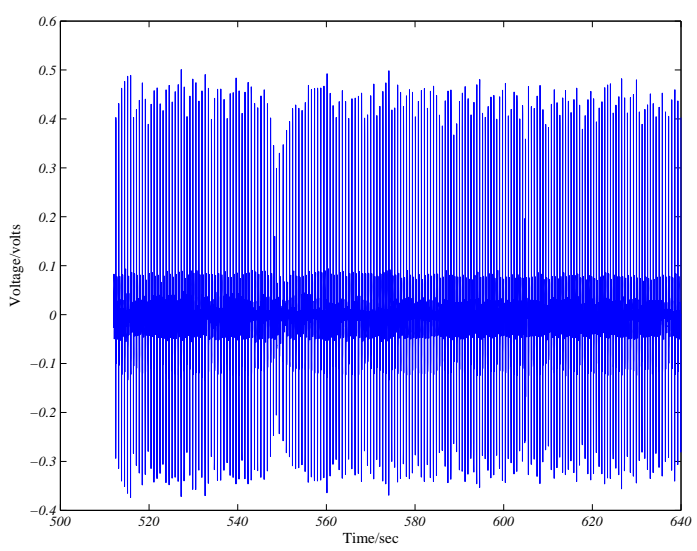

(g) Red-hig light

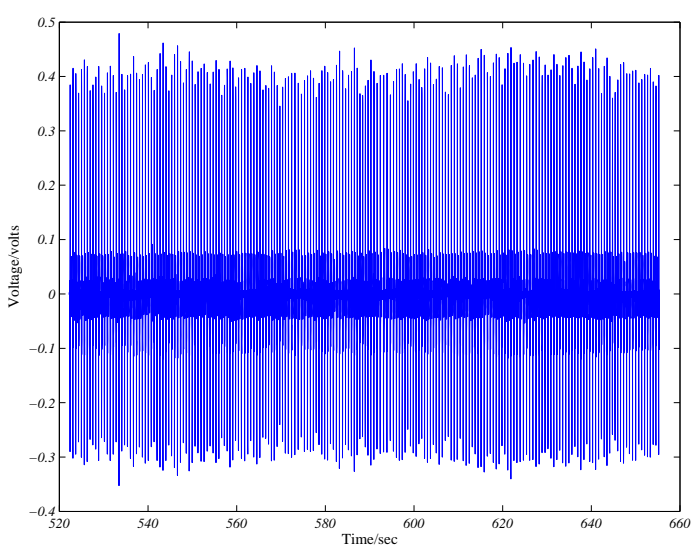

(f) Red-low light

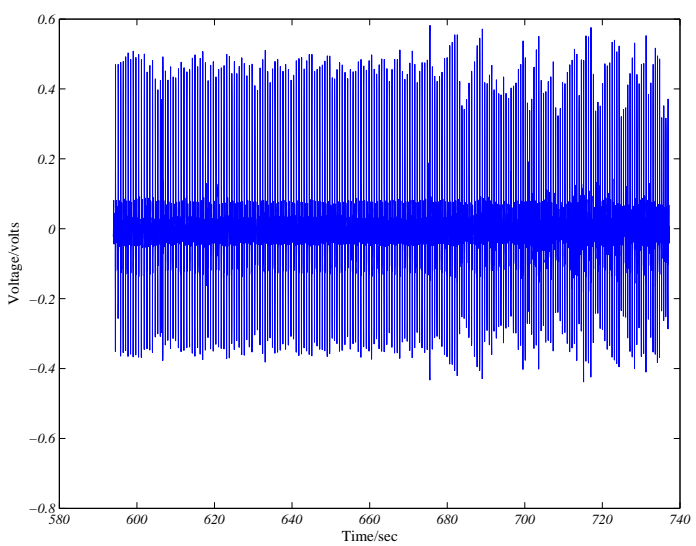

(h) Natural light

Figure A.15: Participant 15 - ECG Recordings for participant 15 for all colour lights 
APPENDIX B

SC Recordings 


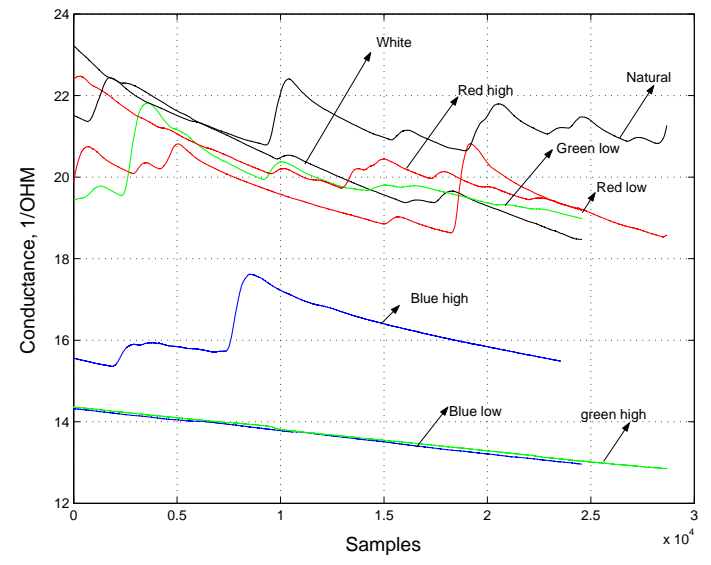

(a) Participant 1

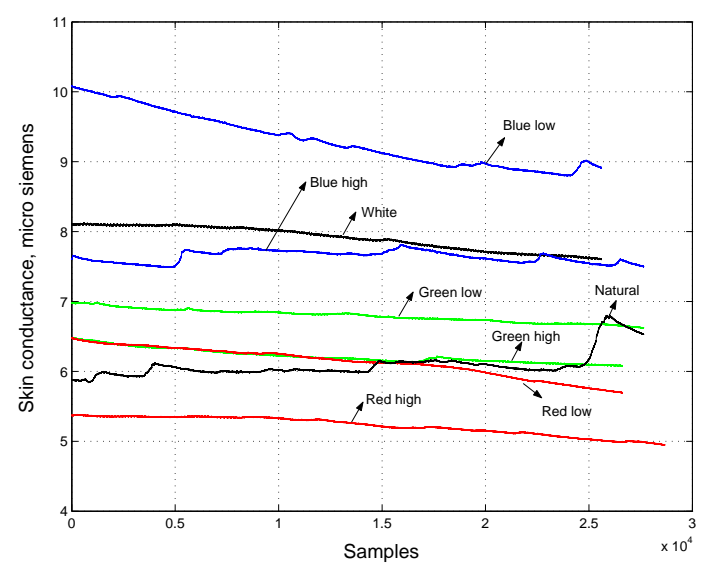

(b) Participant 2

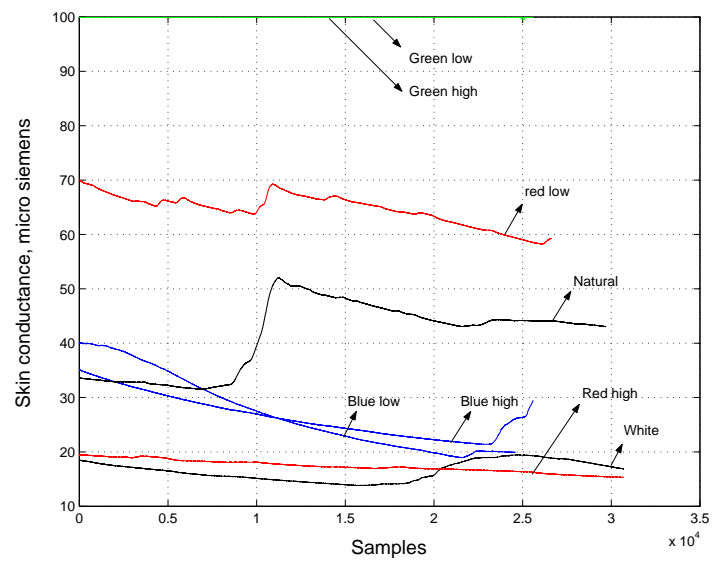

(c) Participant 3

Figure B.1: SC for each participant under all colour lights 


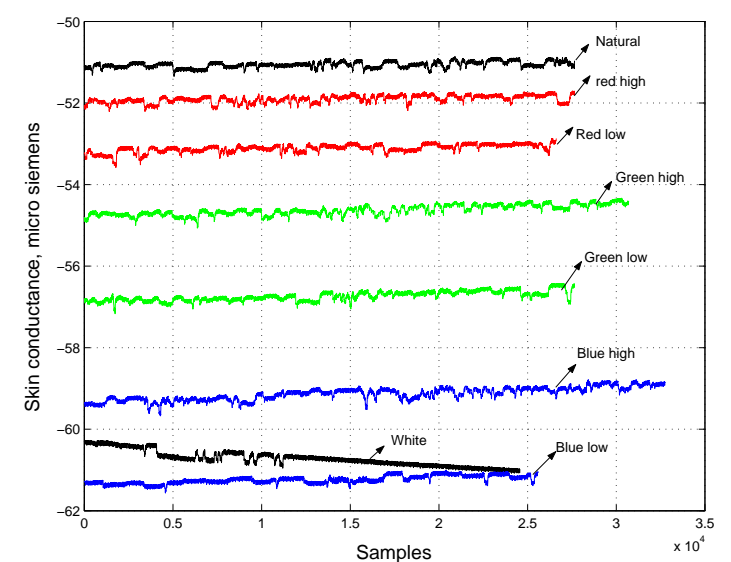

(a) Participant 4

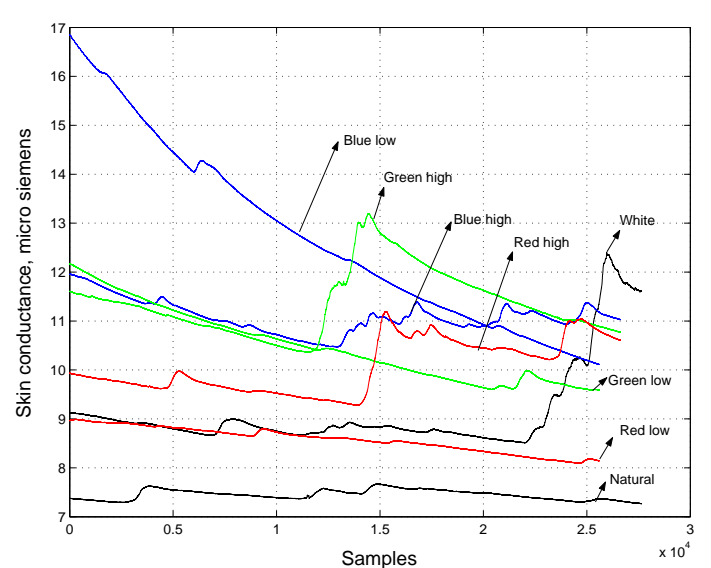

(b) Participant 5

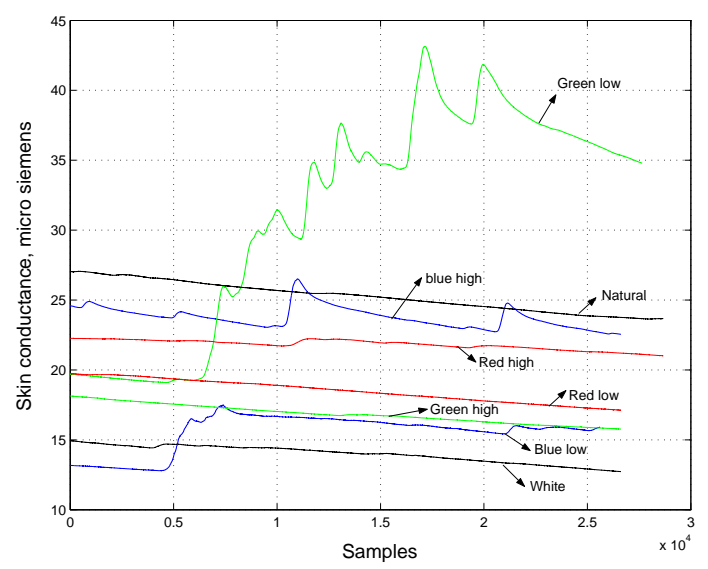

(c) Participant 6

Figure B.2: SC for each participant under all colour lights 


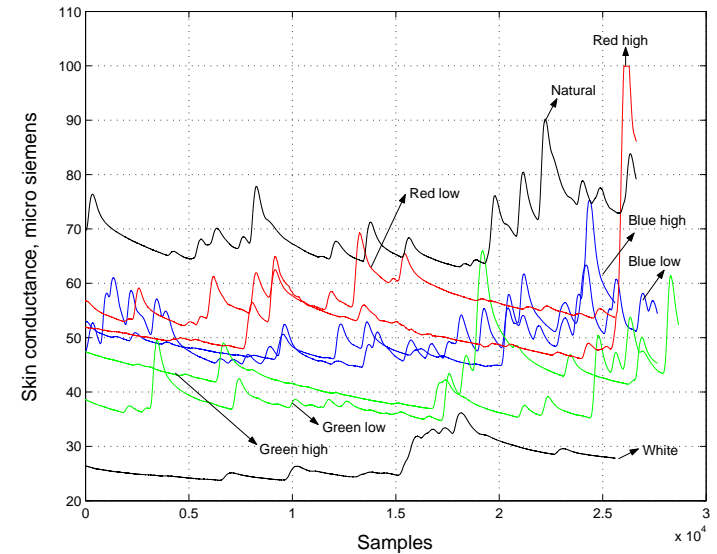

(a) Participant 7

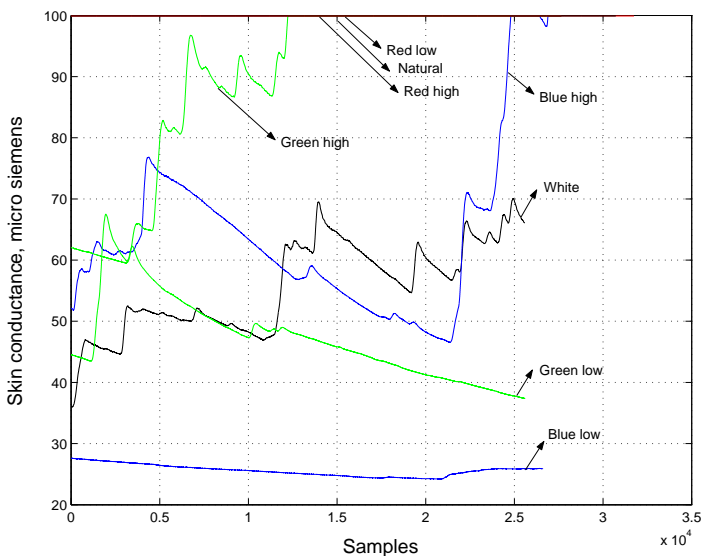

(b) Participant 8

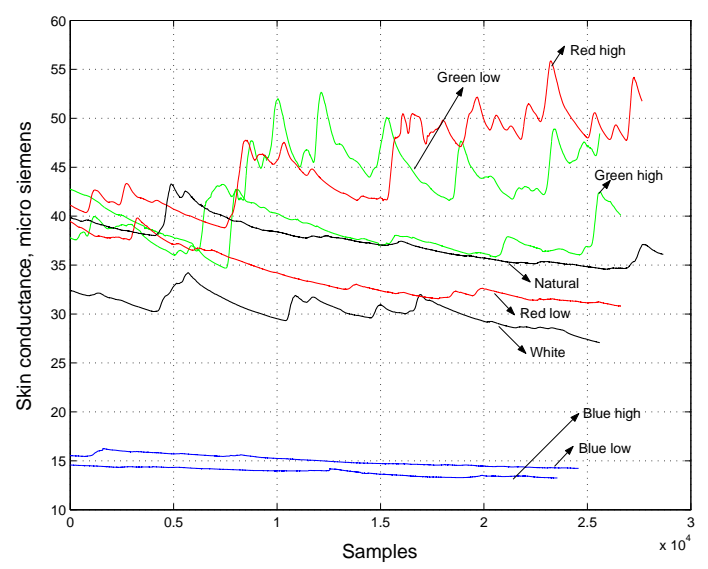

(c) Participant 9

Figure B.3: SC for each participant under all colour lights 


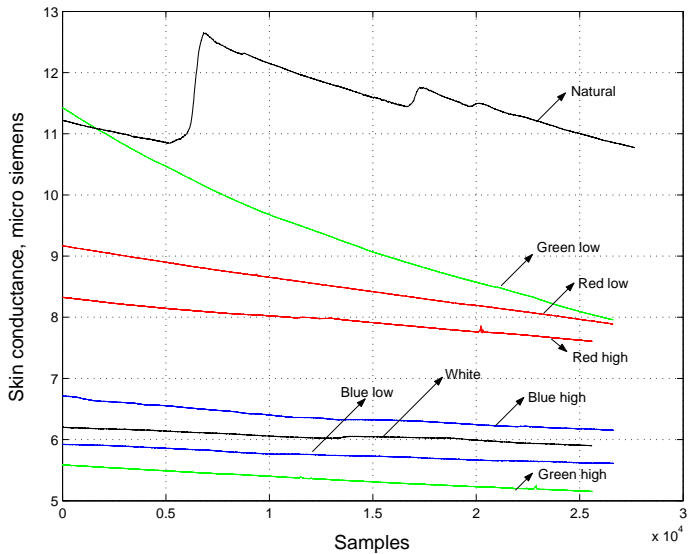

(a) Participant 10

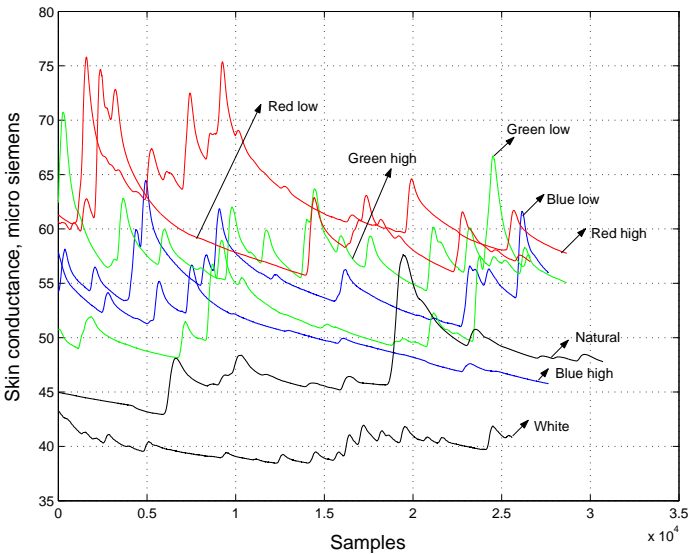

(b) Participant 11

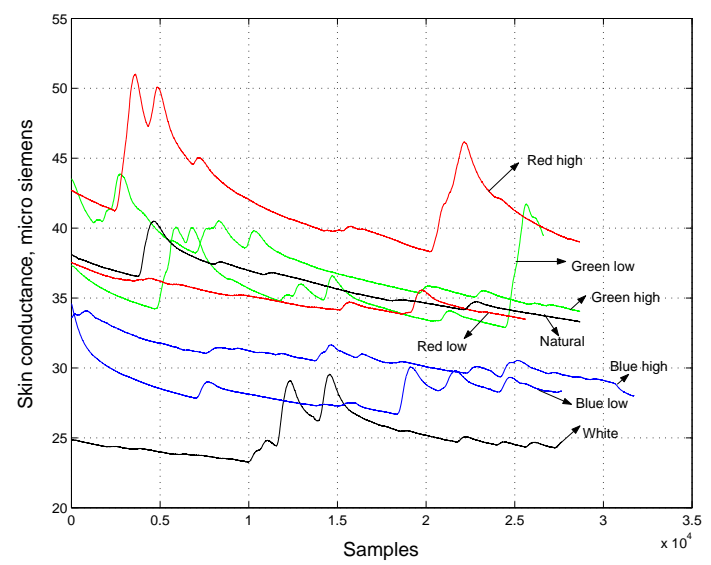

(c) Participant 12

Figure B.4: SC for each participant under all colour lights 


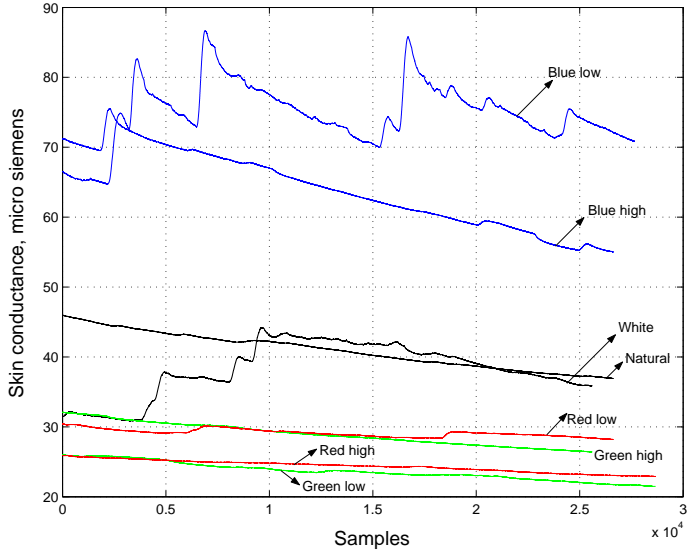

(a) Participant 13

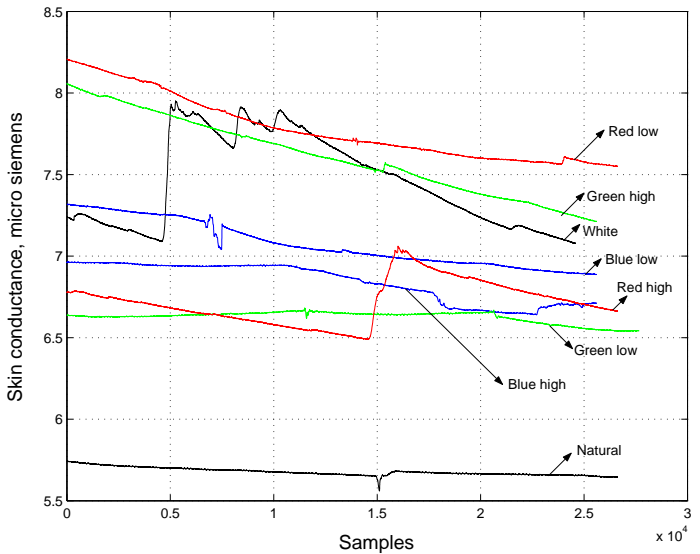

(b) Participant 14

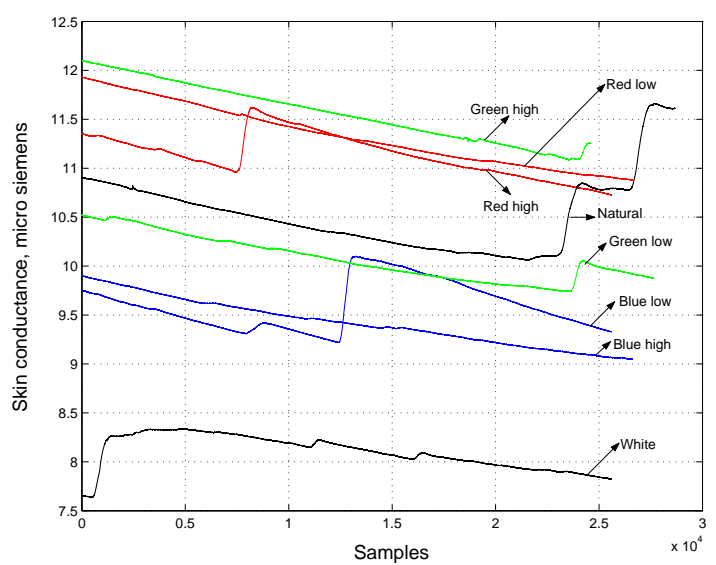

(c) Participant 15

Figure B.5: SC for each participant under all colour lights 
appendix C

HR Charts 


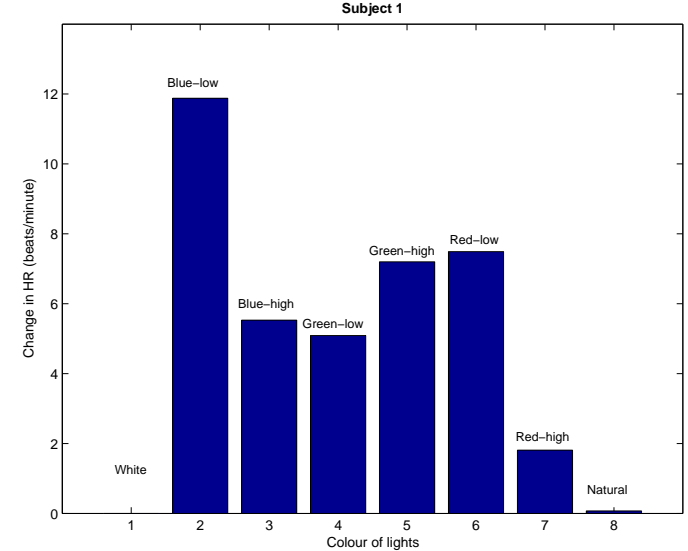

(a) Participant 1

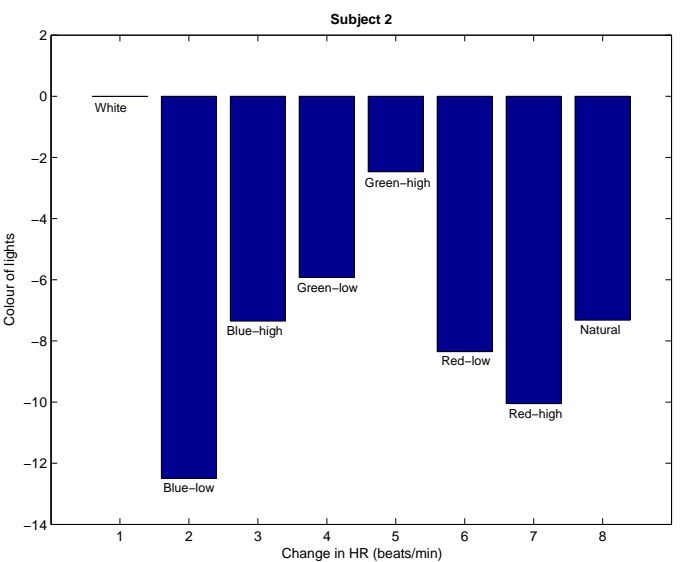

(b) Participant 2

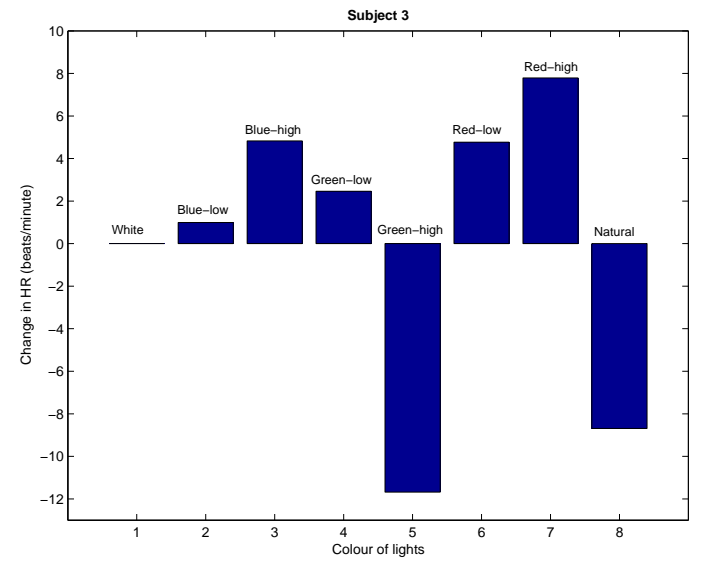

(c) Participant 3

Figure C.1: Change in HR for each participant under all colour lights 


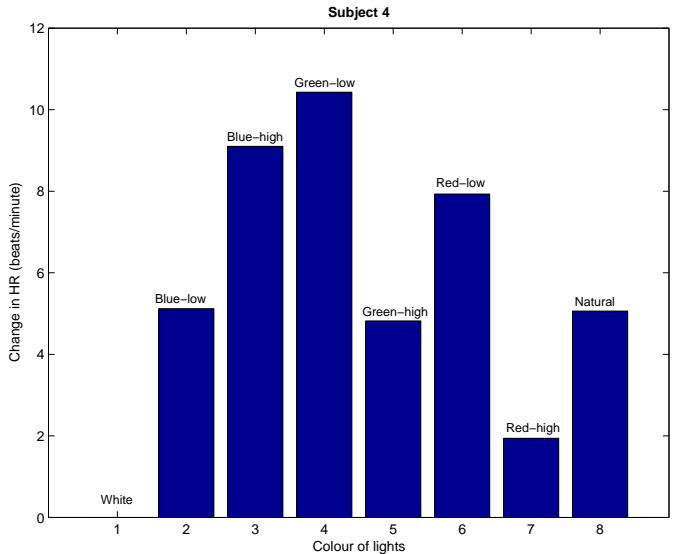

(a) Participant 4

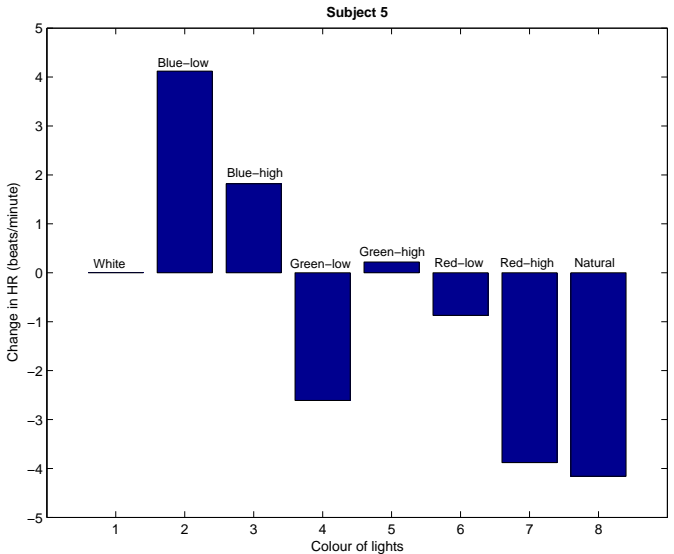

(b) Participant 5

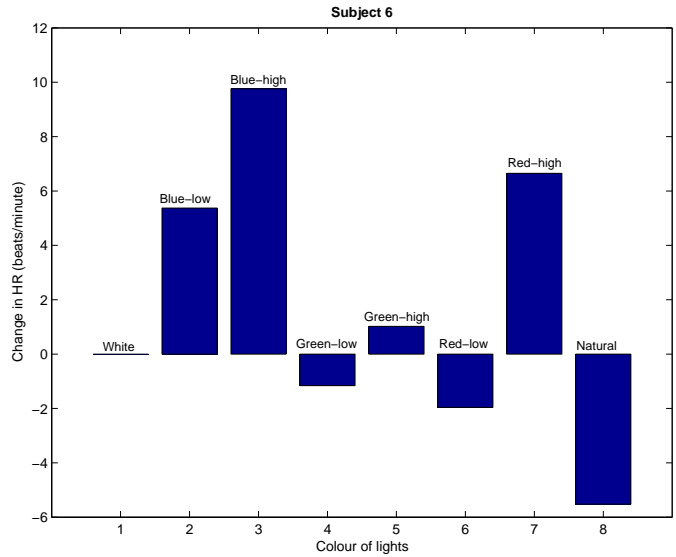

(c) Participant 6

Figure C.2: Change in HR for each participant under all colour lights 


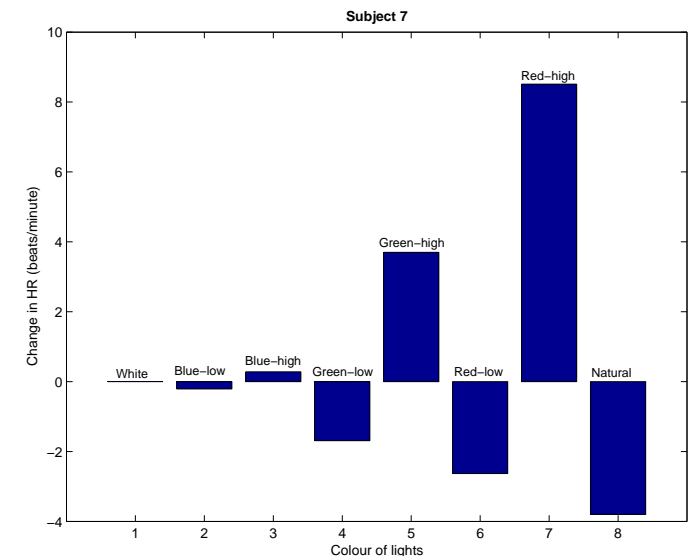

(a) Participant 7

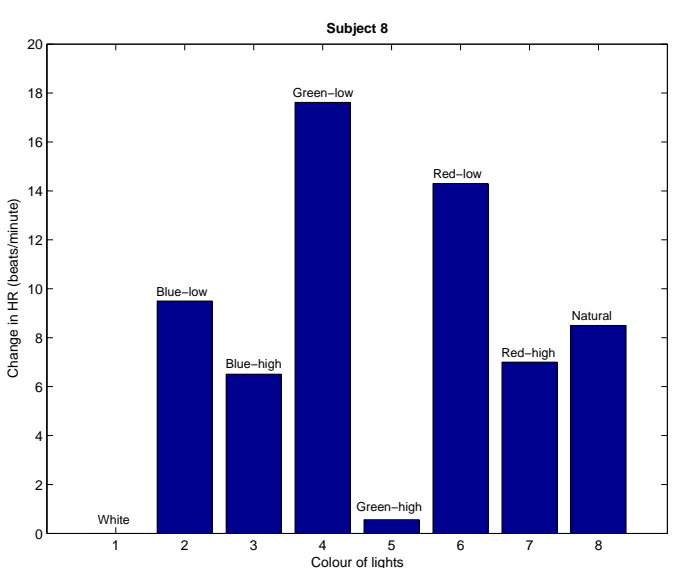

(b) Participant 8

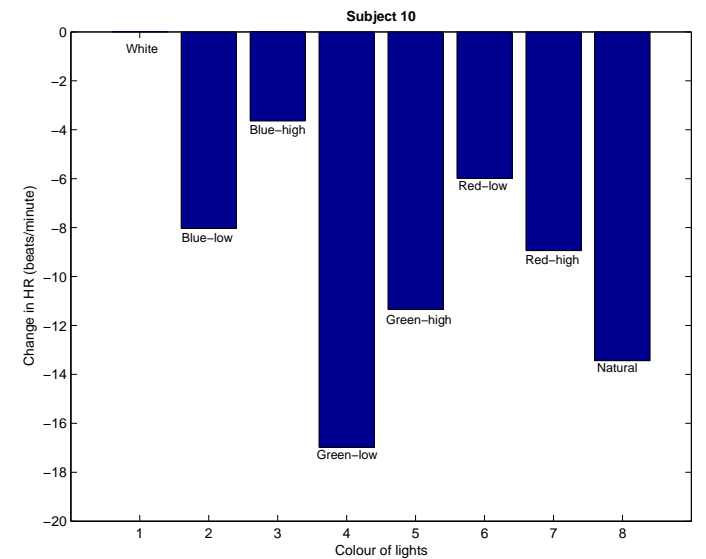

(c) Participant 9

Figure C.3: Change in HR for each participant under all colour lights 


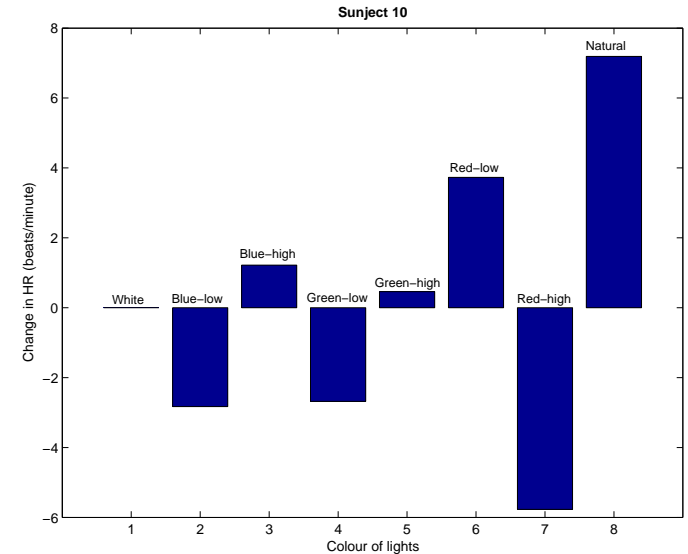

(a) Participant 10

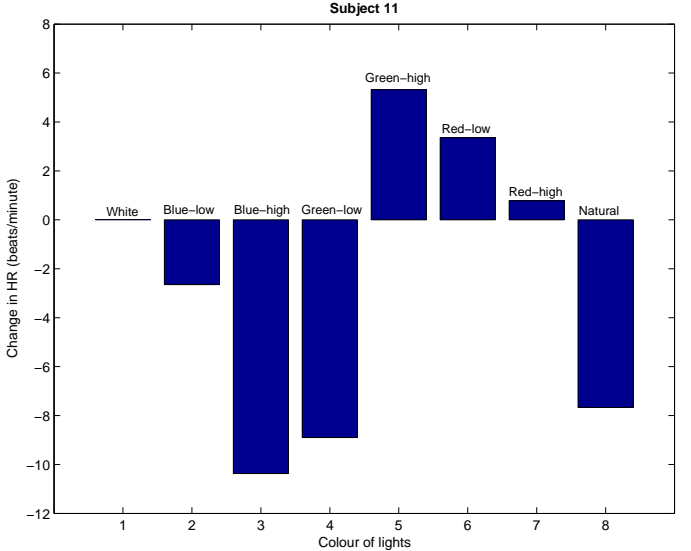

(b) Participant 11

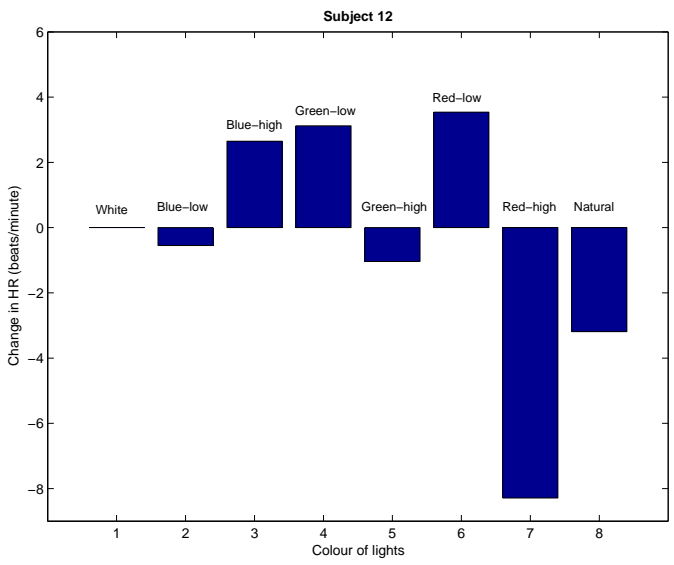

(c) Participant 12

Figure C.4: Change in HR for each participant under all colour lights 


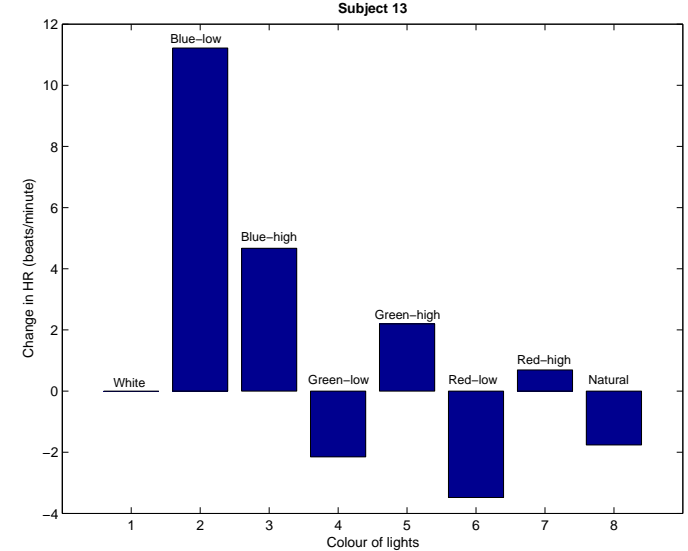

(a) Participant 13

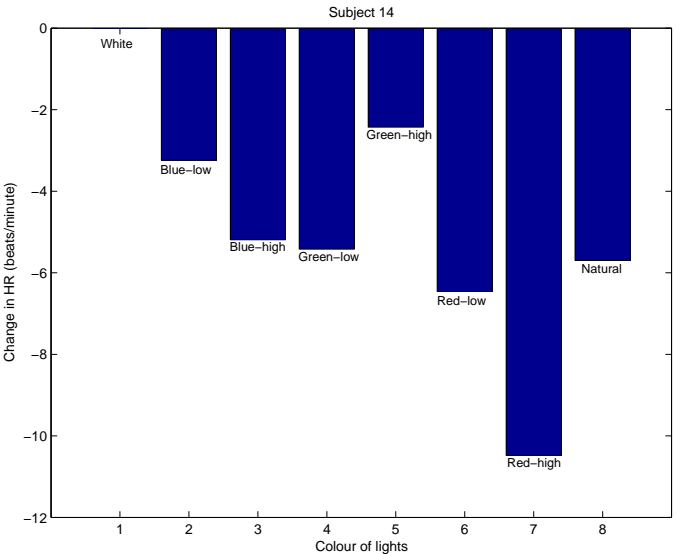

(b) Participant 14

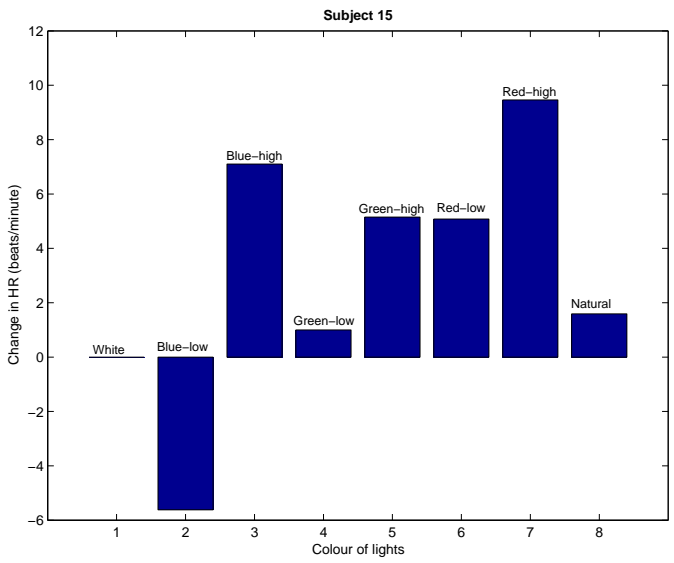

(c) Participant 15

Figure C.5: Change in HR for each participant under all colour lights 
APPENDIX D

$\begin{array}{r}\text { SC Charts } \\ \hline\end{array}$ 


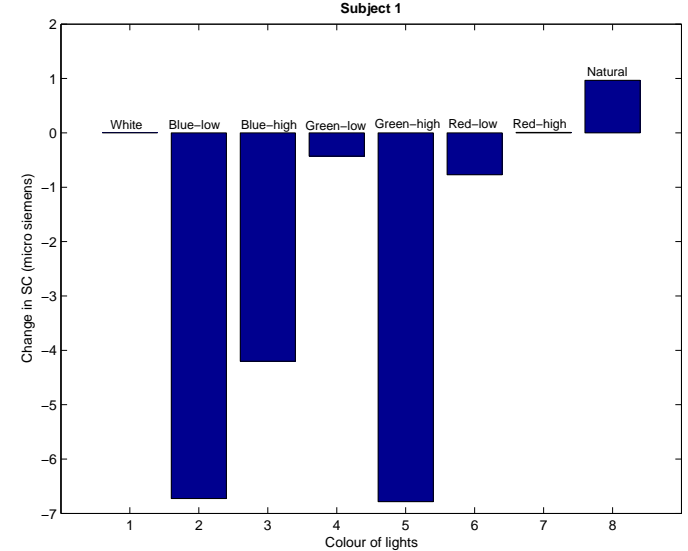

(a) Participant 1

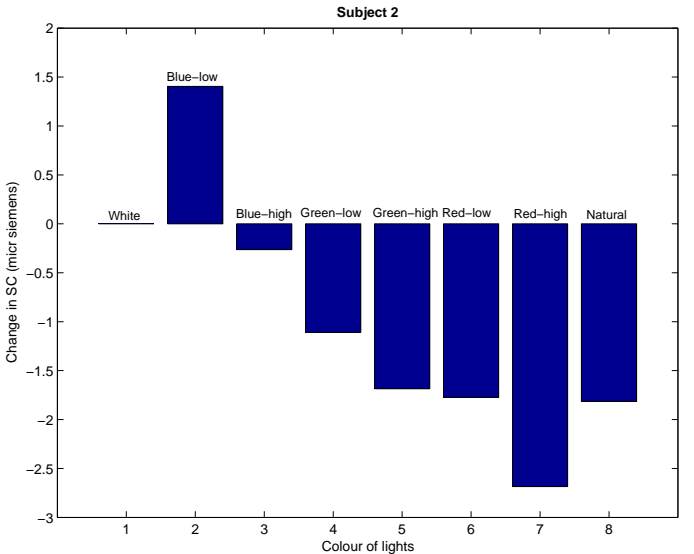

(b) Participant 2

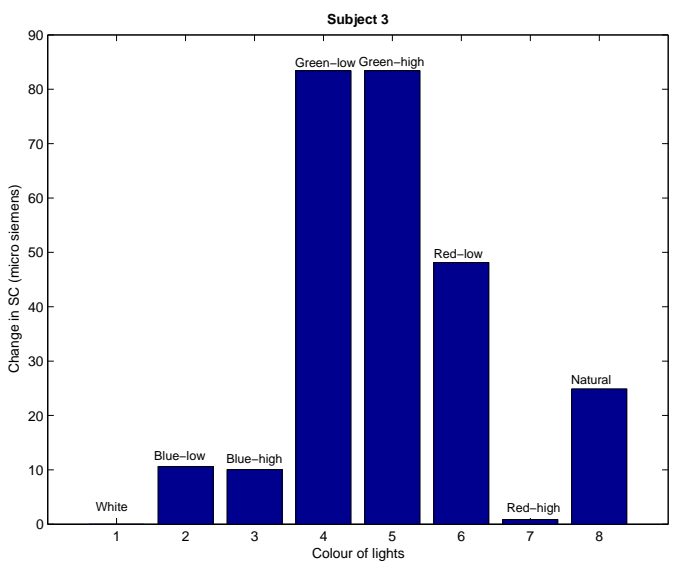

(c) Participant 3

Figure D.1: Change in SC for each participant under all colour lights 


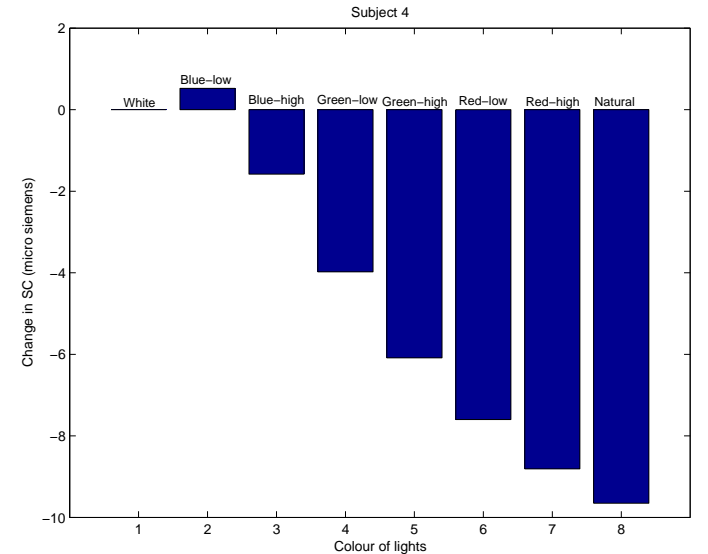

(a) Participant 4

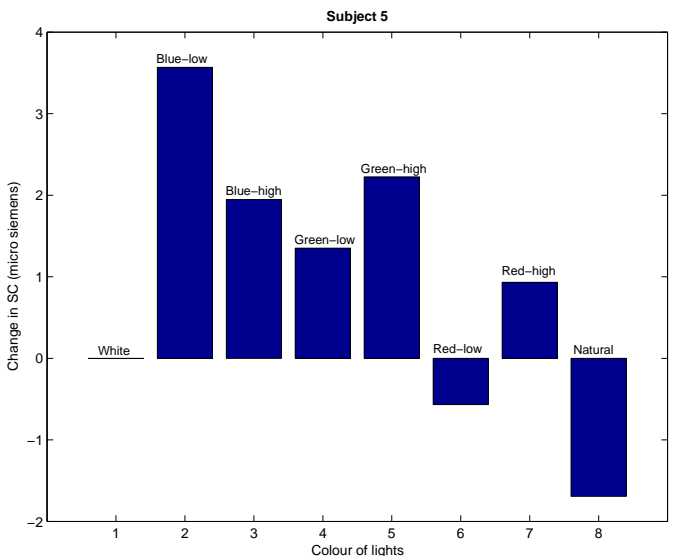

(b) Participant 5

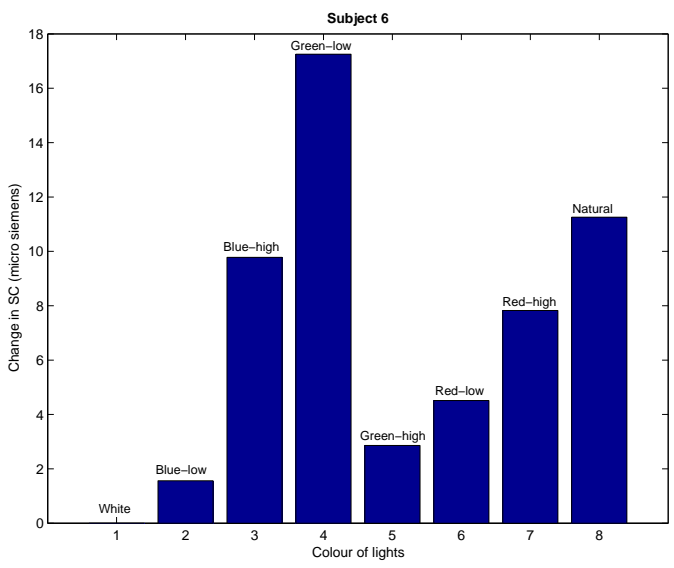

(c) Participant 6

Figure D.2: Change in SC for each participant under all colour lights 


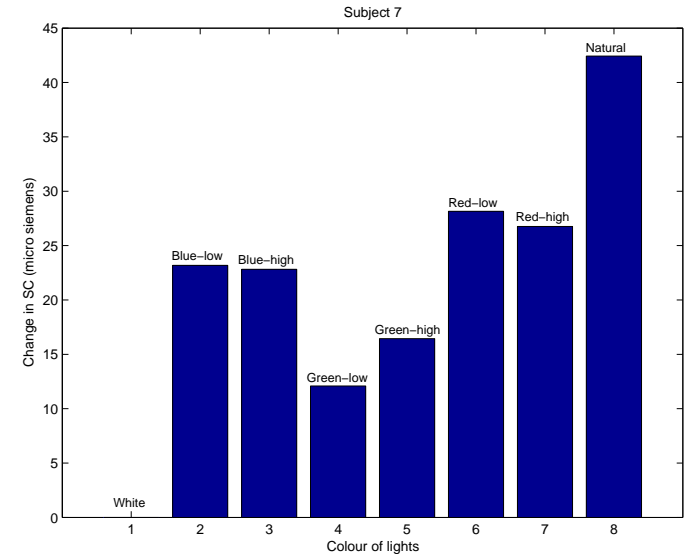

(a) Participant 7

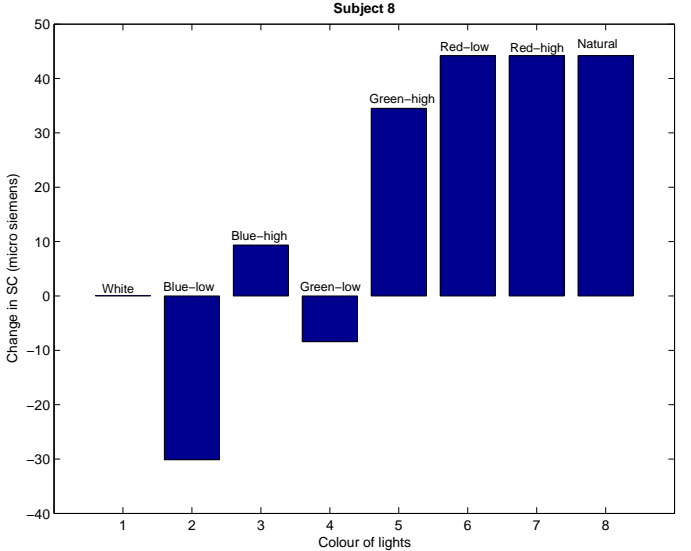

(b) Participant 8

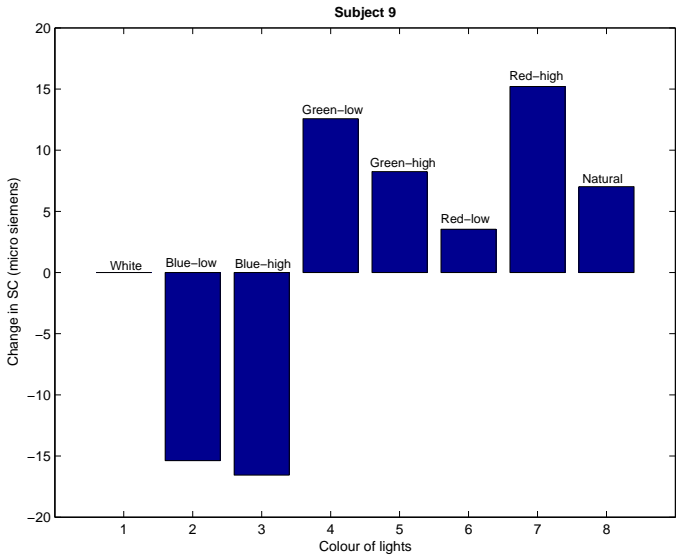

(c) Participant 9

Figure D.3: Change in SC for each participant under all colour lights 


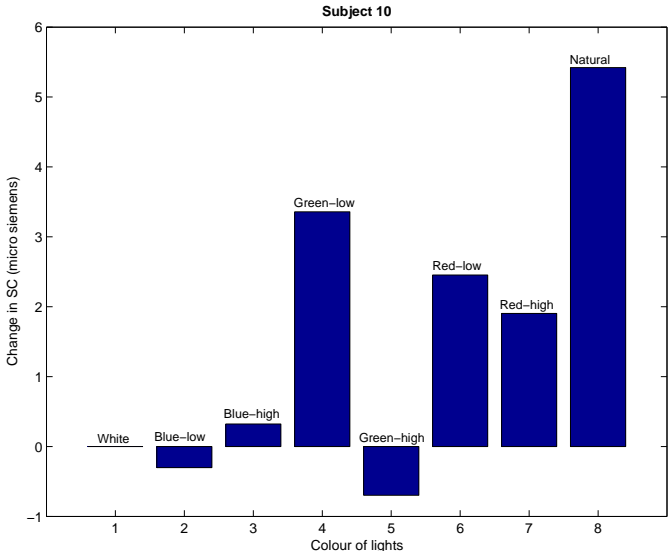

(a) Participant 10

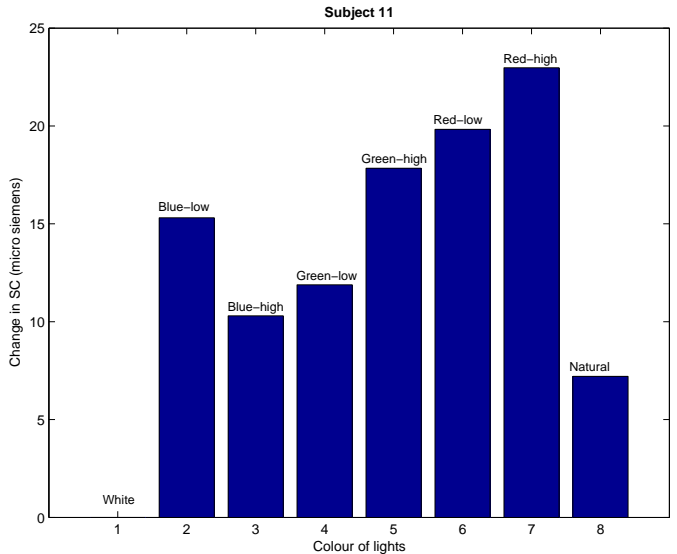

(b) Participant 11

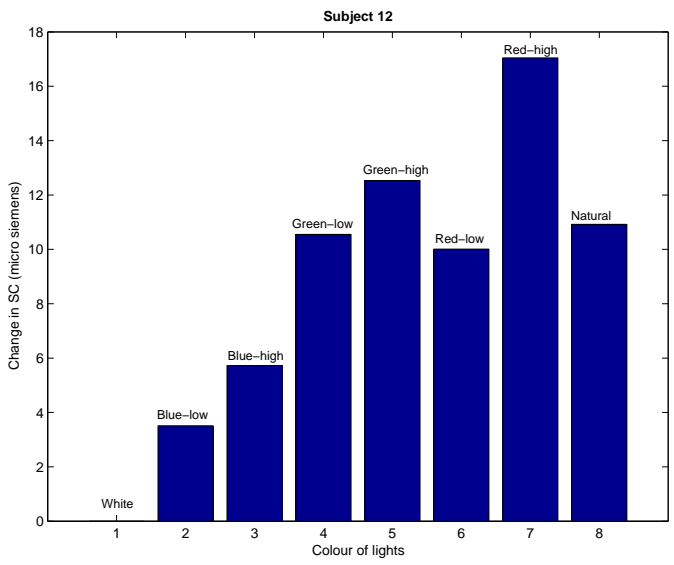

(c) Participant 12

Figure D.4: Change in SC for each participant under all colour lights 


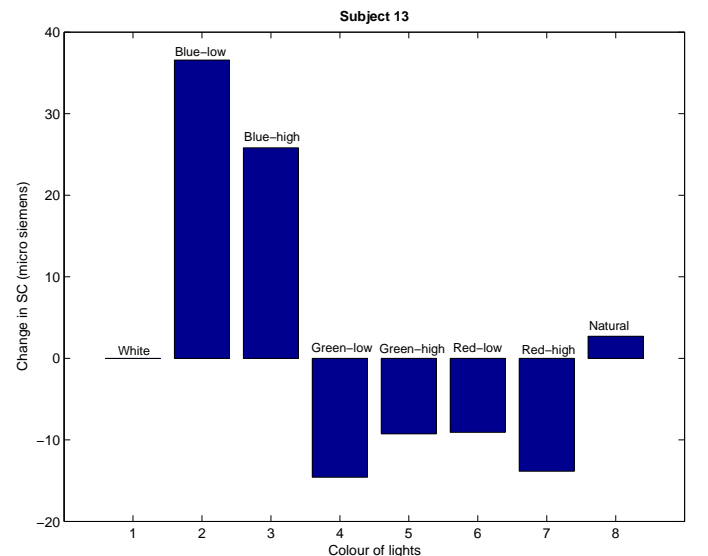

(a) Participant 13

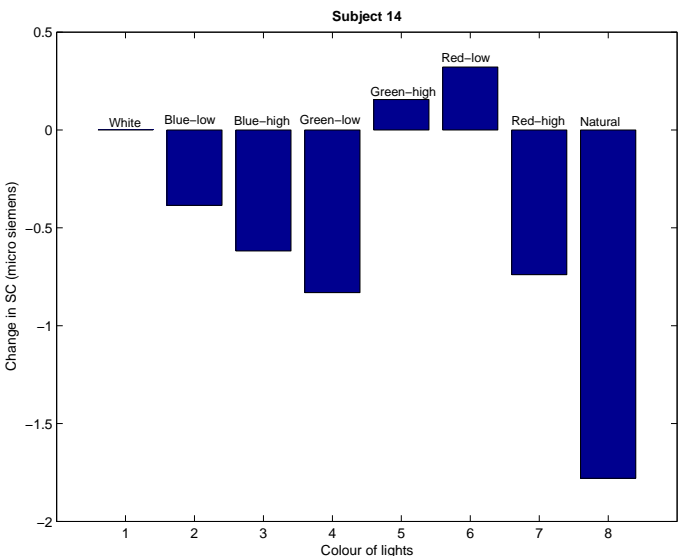

(b) Participant 14

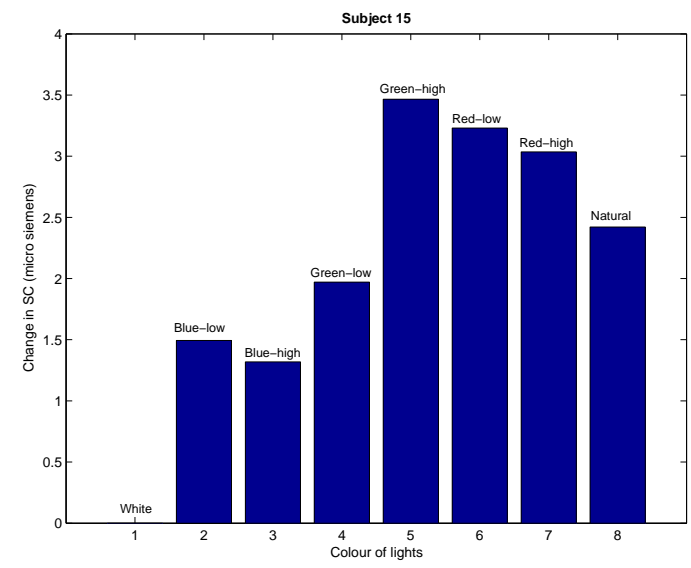

(c) Participant 15

Figure D.5: Change in SC for each participant under all colour lights 
APPENDIX E

Arousal Charts 


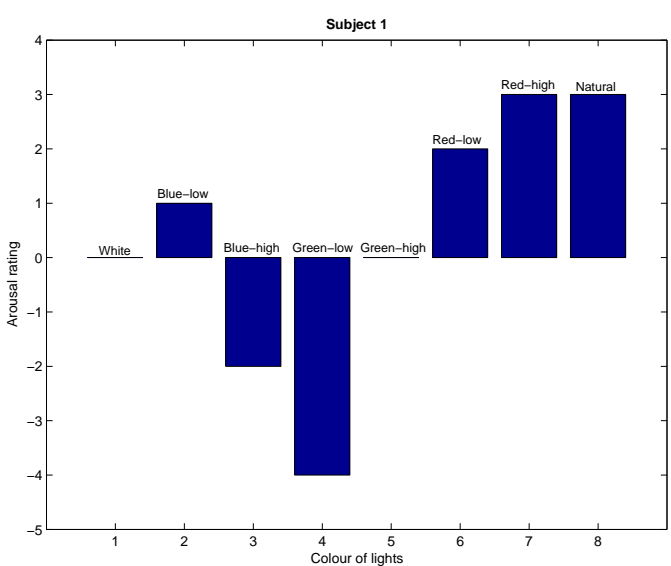

(a) Participant 1

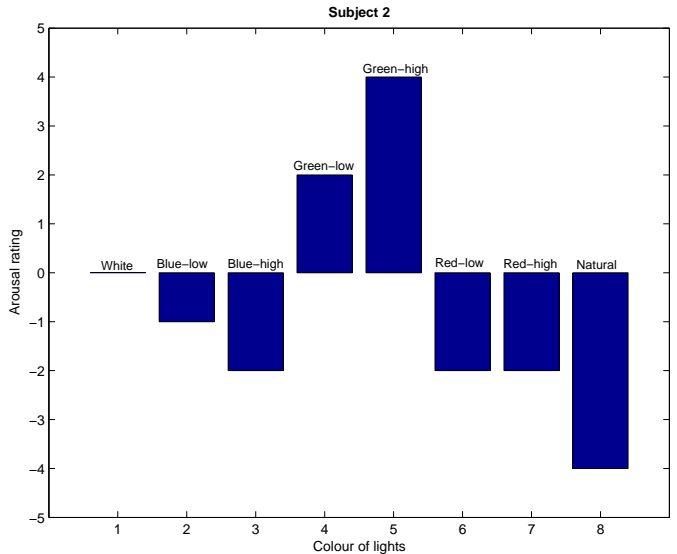

(b) Participant 2

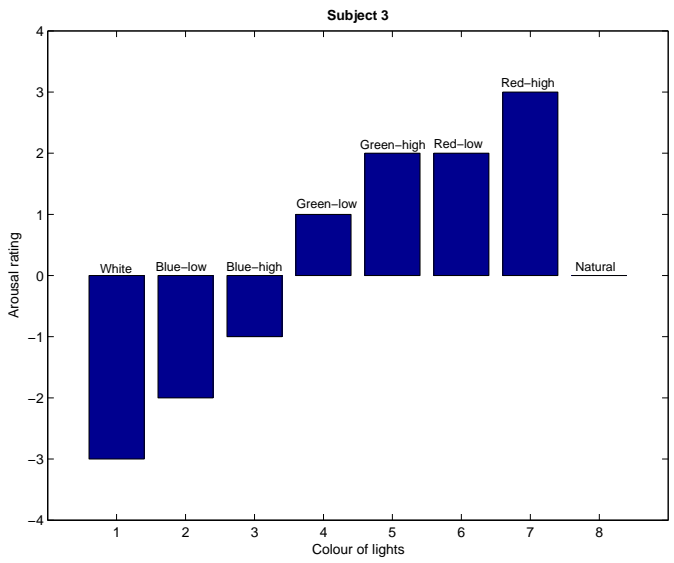

(c) Participant 3

Figure E.1: Arousal of each participant under all colour lights 


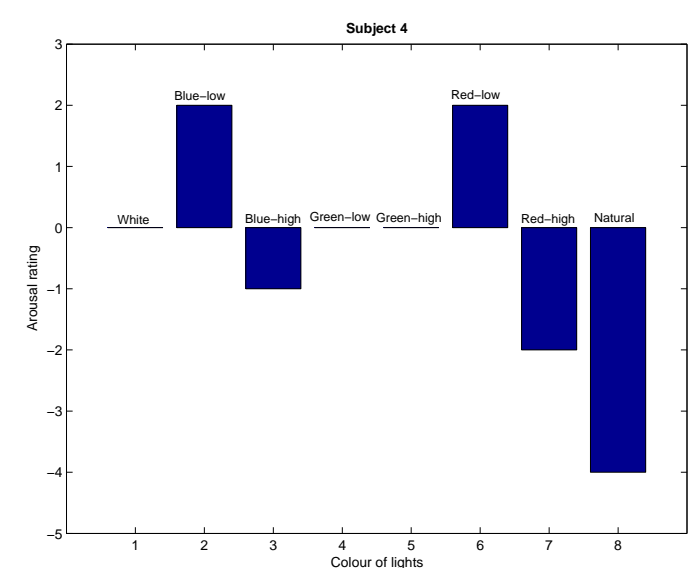

(a) Participant 4

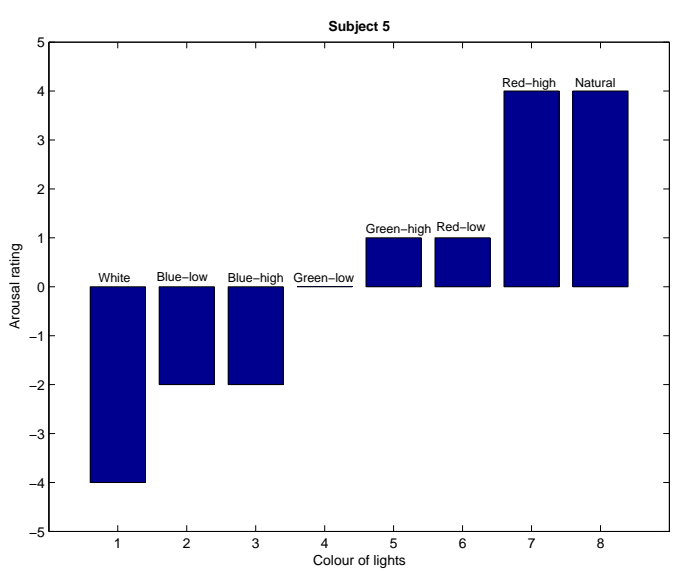

(b) Participant 5

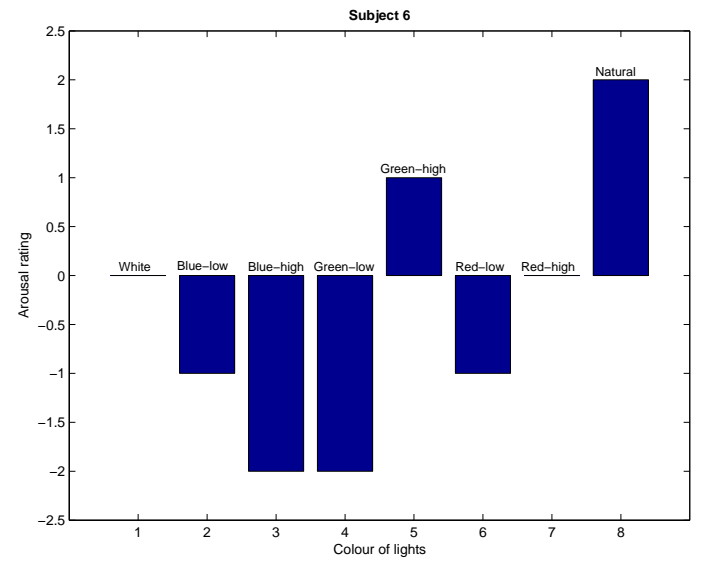

(c) Participant 6

Figure E.2: Arousal of each participant under all colour lights 


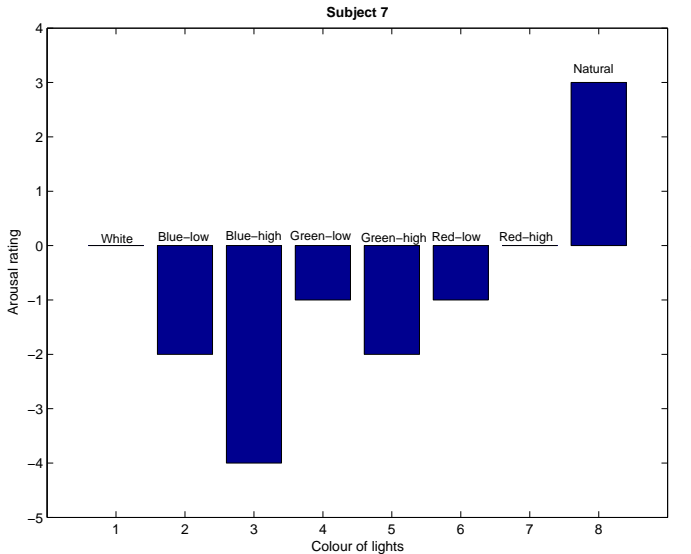

(a) Participant 7

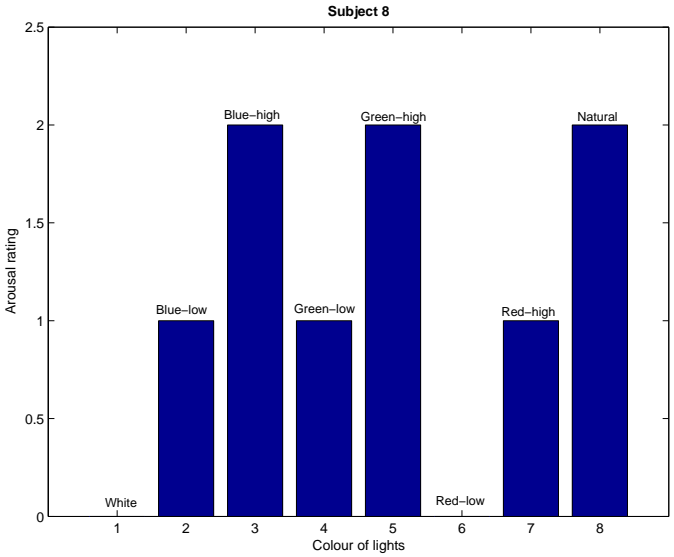

(b) Participant 8

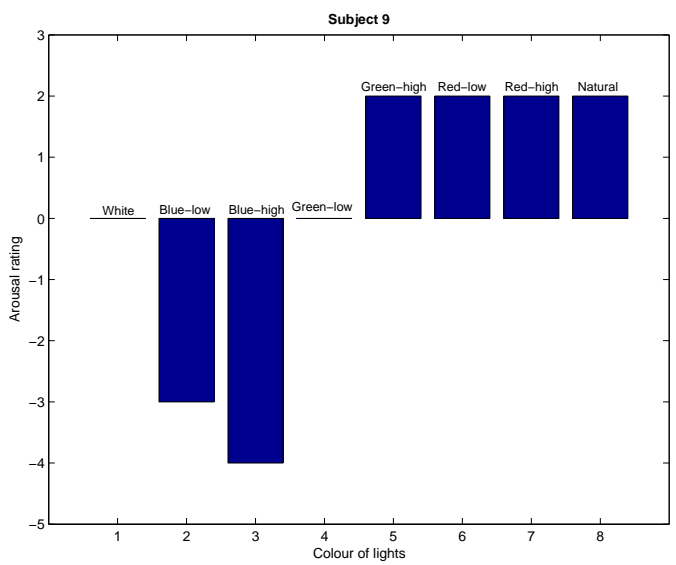

(c) Participant 9

Figure E.3: Arousal of each participant under all colour lights 


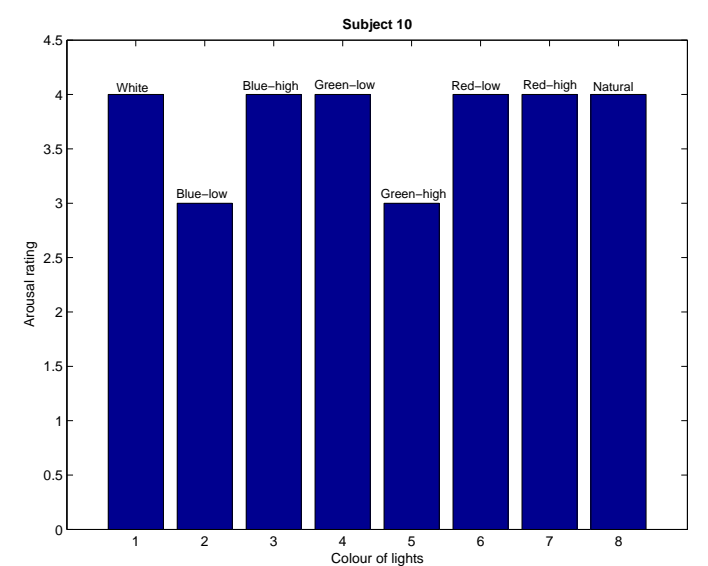

(a) Participant 10

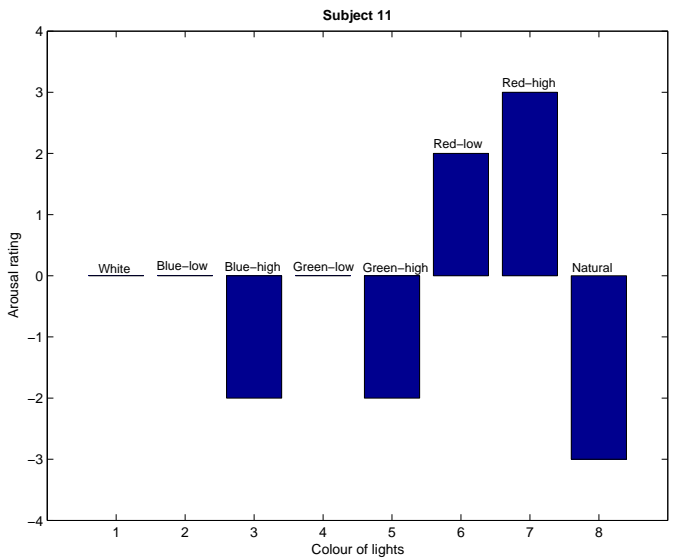

(b) Participant 11

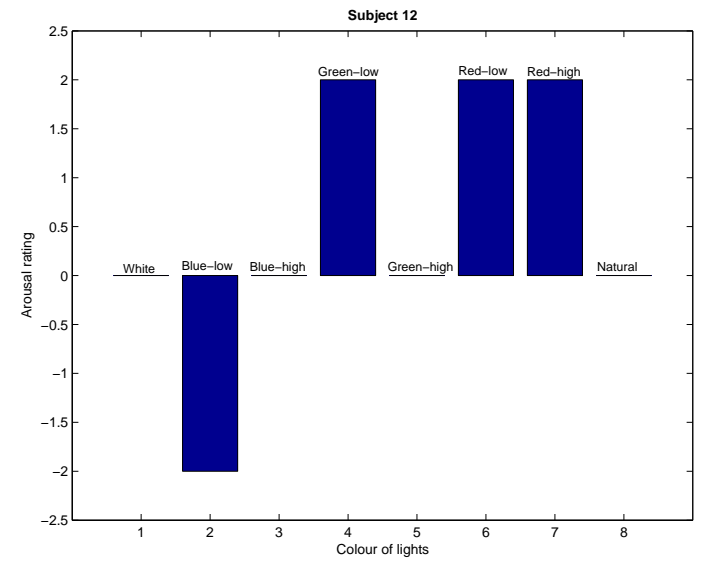

(c) Participant 12

Figure E.4: Arousal of each participant under all colour lights 


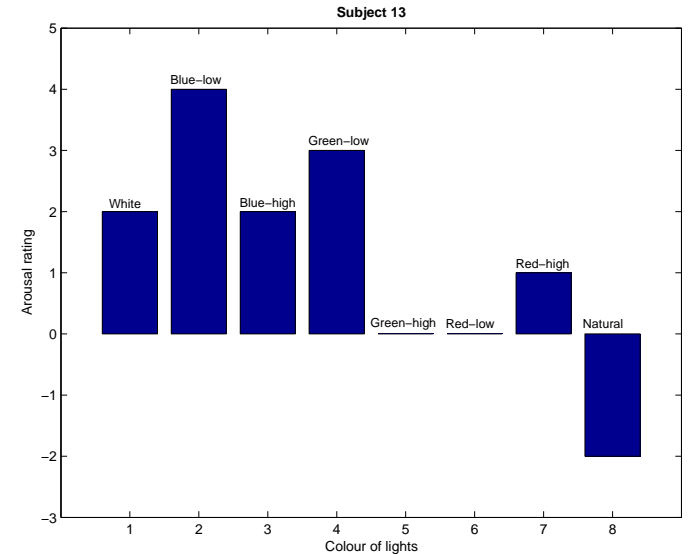

(a) Participant 13

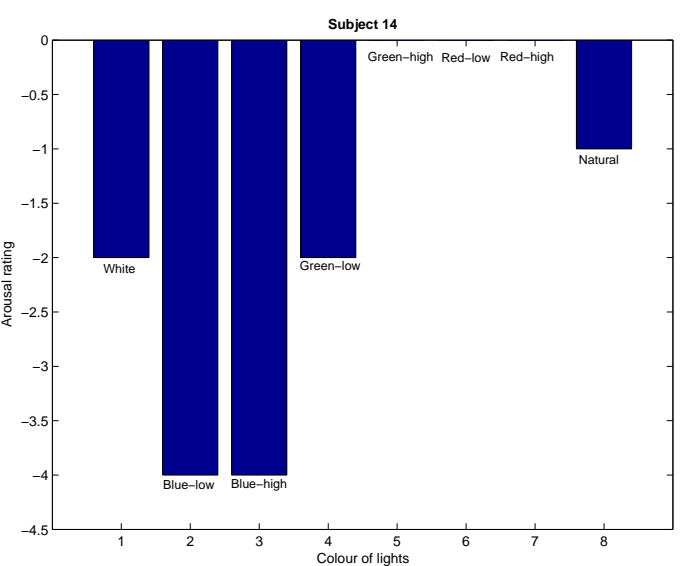

(b) Participant 14

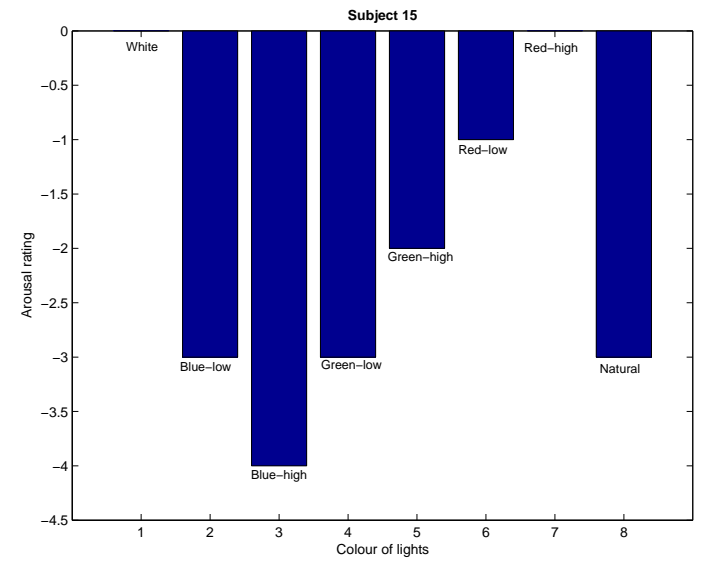

(c) Participant 15

Figure E.5: Arousal of each participant under all colour lights 
APPENDIX F

Valence Charts 


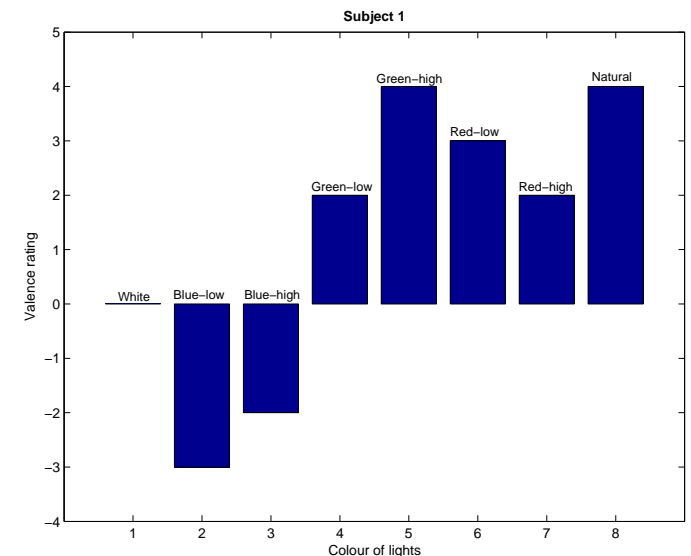

(a) Participant 1

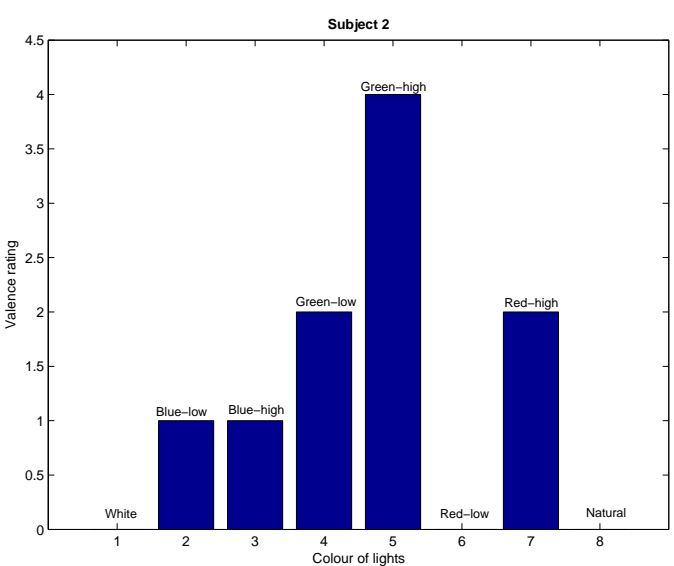

(b) Participant 2

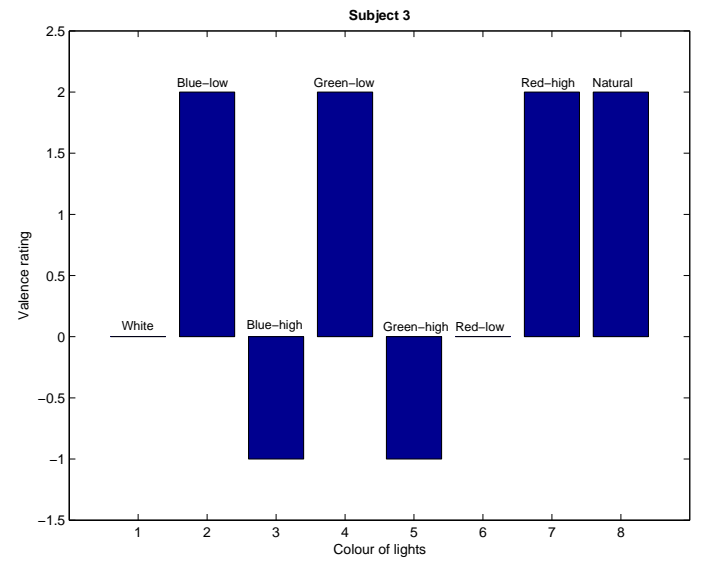

(c) Participant 3

Figure F.1: Valence of each participant under all colour lights 


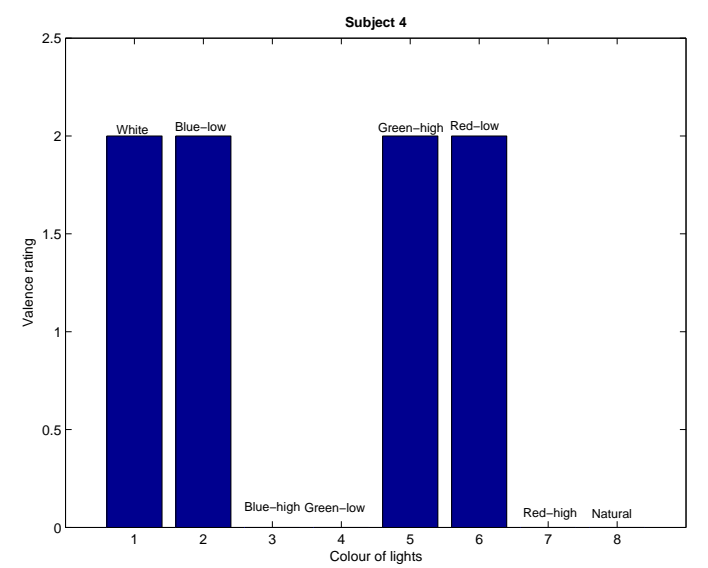

(a) Participant 4

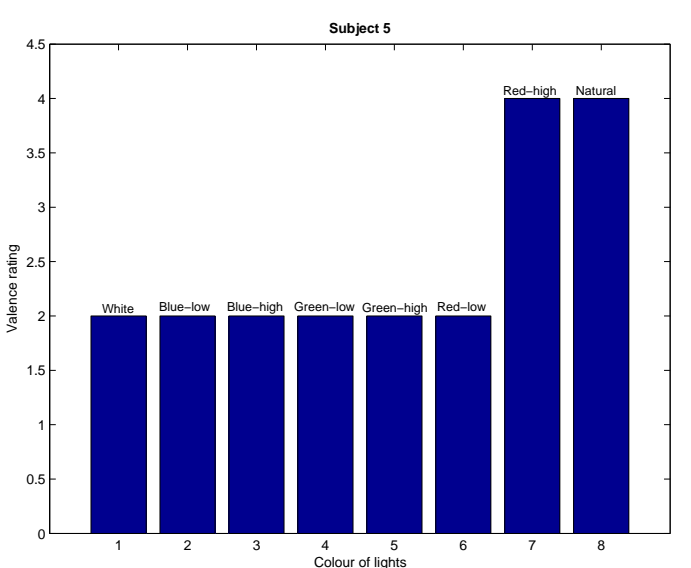

(b) Participant 5

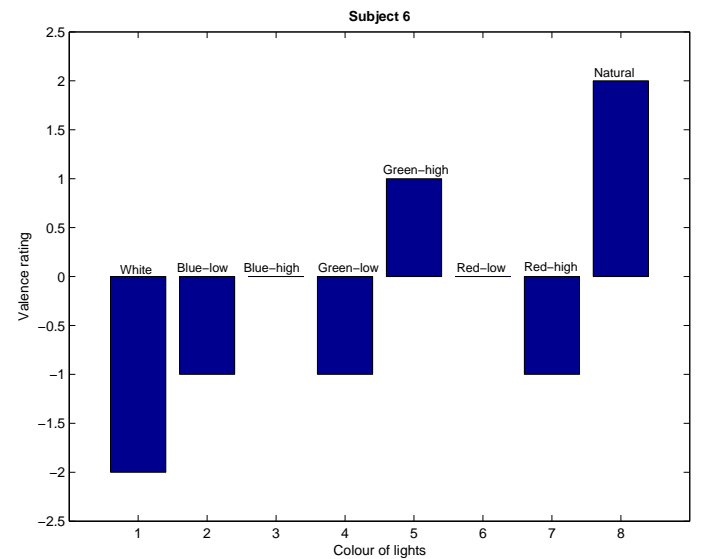

(c) Participant 6

Figure F.2: Valence of each participant under all colour lights 


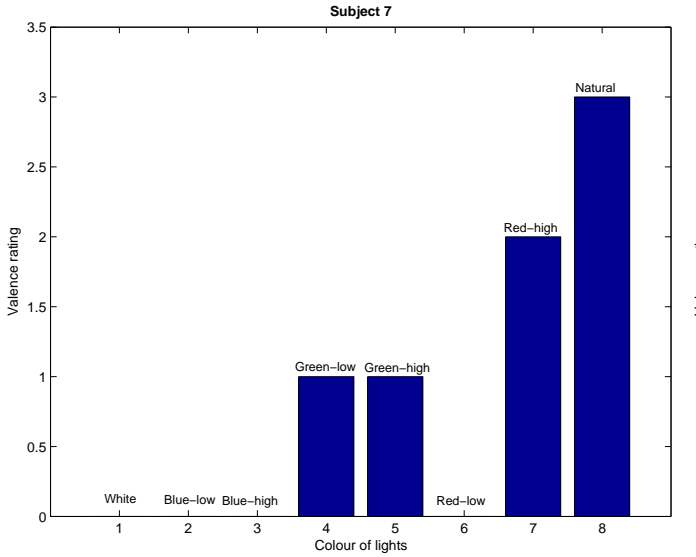

(a) Participant 7

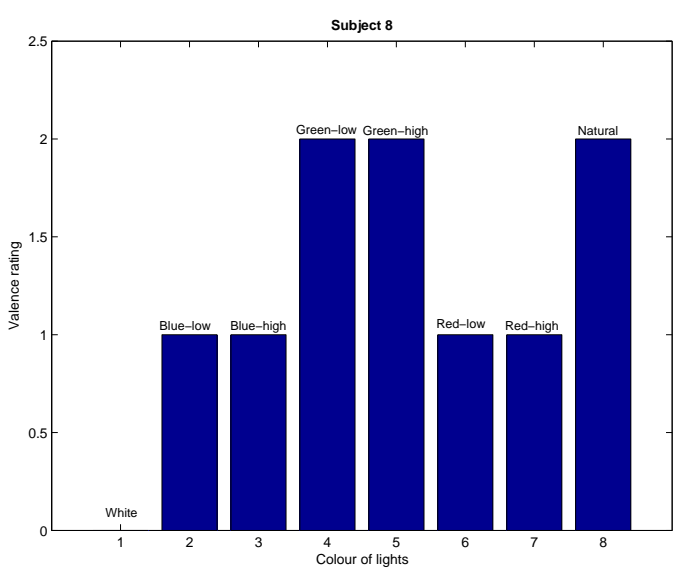

(b) Participant 8

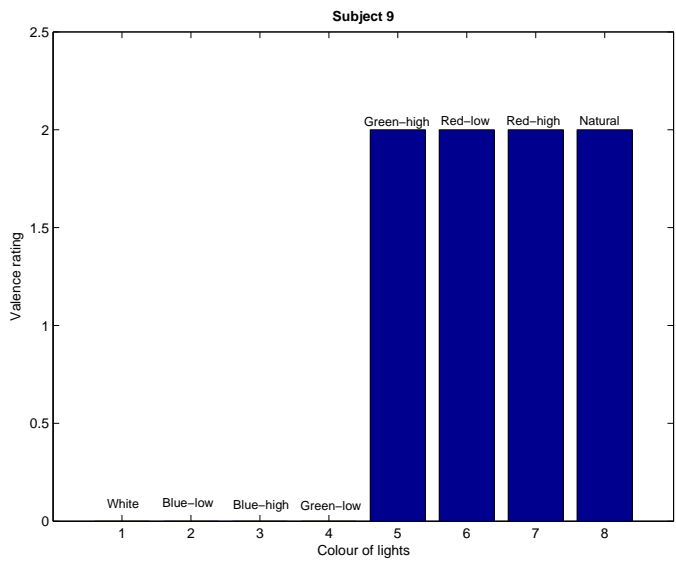

(c) Participant 9

Figure F.3: Valence of each participant under all colour lights 


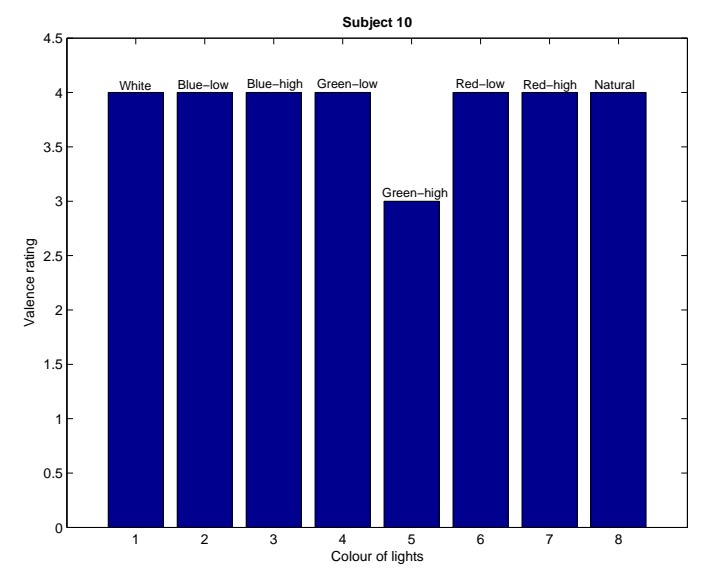

(a) Participant 10

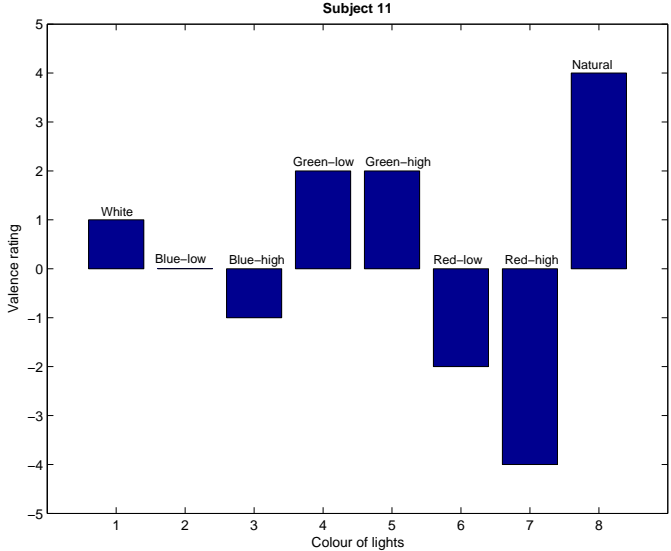

(b) Participant 11

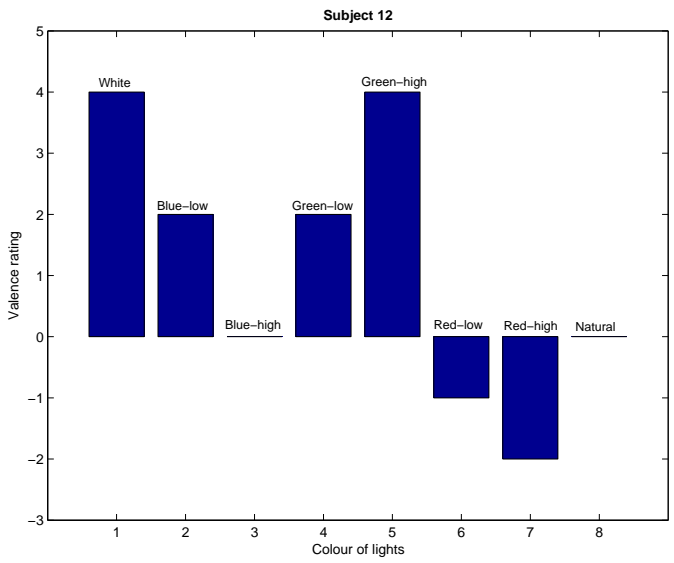

(c) Participant 12

Figure F.4: Valence of each participant under all colour lights 


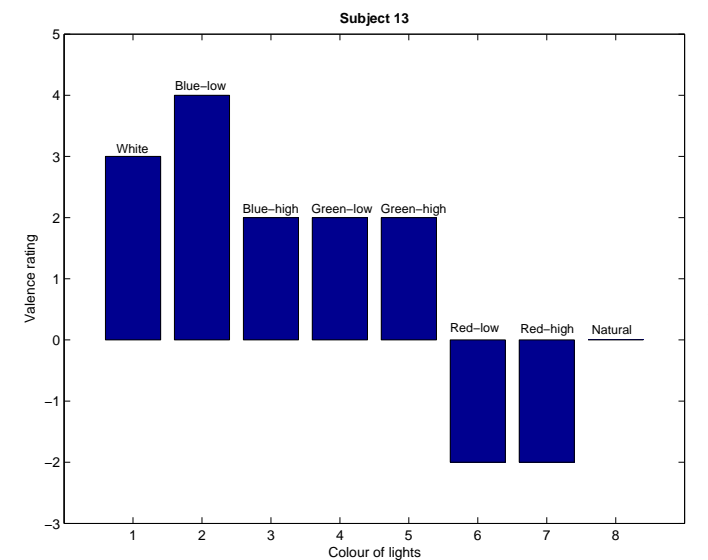

(a) Participant 13

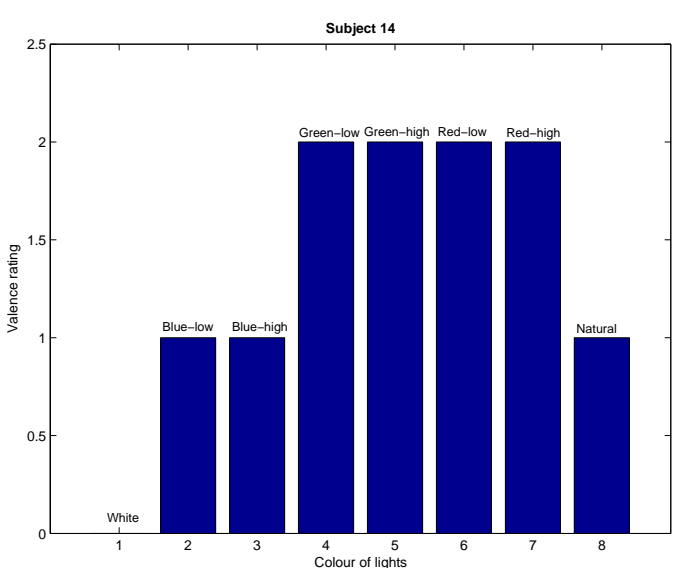

(b) Participant 14

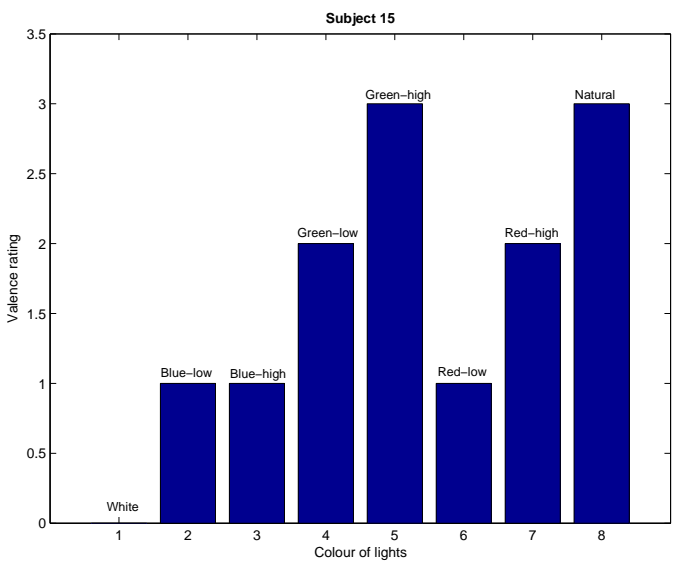

(c) Participant 15

Figure F.5: Valence of each participant under all colour lights 


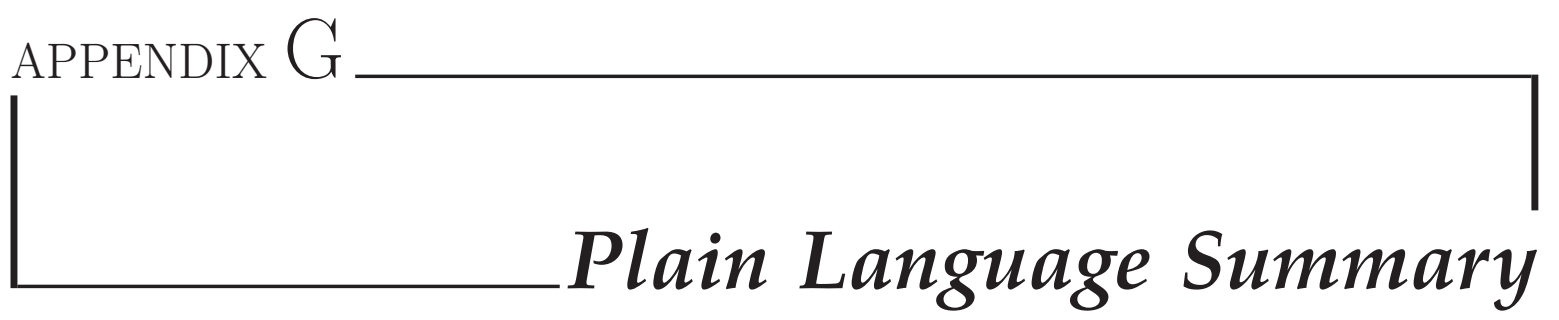

Nadeen Abbas

School of Electrical \& Computer Engineering,

RMIT University

Email: s3060446@student.rmit.edu.au

Dear Sir/Madam,

I would like to invite you to participate in an experiment aimed at investigating the psychological and emotional effects of light intensity and colour on people. These experiments will be conducted by myself, Nadeen Abbas, as part of my masters programme at the School of Electrical \& Computer Engineering, RMIT University. The research will be supervised by DR Dinesh Kant Kumar and DR Neil Mclachlan at RMIT. The outcomes of this study are expected to address the architects' choice of light intensity and colour. This will help architects base their choices of light intensity and colour on scientific facts which will improve the users' performance in the space.

- Research Title: The psychological and physiological effects of light and colour on space users.

- Description of experiments: You will be seated in a room, your heart rate and skin conductance will be recorded as the light intensity and colour of the room changes. For skin 
conductance measurements, a single sensor strap will be placed around your two fingers. For the heart rate measurements, surface-stick electrode pads will be palced on both your wrists. All electrodes and sensors are approved for clinical use and are extremely safe.

The attendence period required will be 120 minutes.

Participation in the research is purely voluntary. You are welcome, and should, ask for clarification at any time of any aspect that concerns you. You may withdraw consent to participation and discontinue participation at any time. The names of participants will not be recorded to ensure the privacy of data of all participants. The final report of this research may be published in a journal and the participants will be referred to as subject 1 , subject 2 etc. in the publication.

You are welcome to gain access to your data by contacting me.

Please find any contact details above for any queries or expressions of interest to participate in this investigation.

Thank you for your consideration.

Nadeen Abbas 


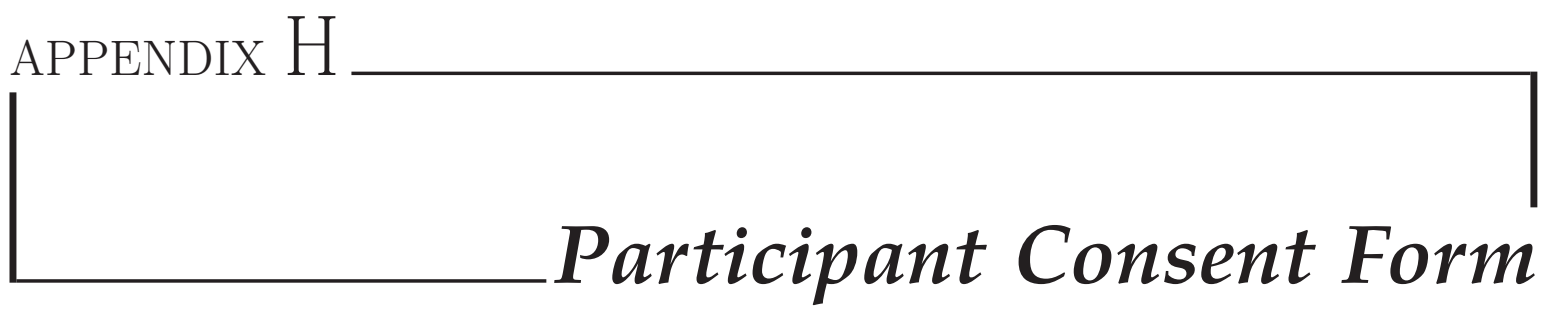

\section{RMIT HUMAN RESEARCH ETHICS COMMITTEE}

Prescribed Consent Form for Persons Participating In Research Projects Involving Tests and / or Medical Procedures.

Faculty of

Department of

Name of the Participant

Project Title

Investigator

\section{Engineering}

Electrical and Computer Engineering

The psychological and physiological effects of light and colour on space users Nadeen Abbas

Phone Number

1. I have received a statement explaining the tests/procedures involved in this project.

2. I consent to participate in the above project, the particulars of which - including details of tests or procedures - have been explained to me.

3. I authorise the investigator or his or her assistant to use with me the tests or procedures referred to in 1 above.

4. I acknowledge that: 
- The possible effects of the tests or procedures have been explained to me to my satisfaction.

- I have been informed that I am free to withdraw from the project at any time and to withdraw any unprocessed data previously supplied (unless follow-up is needed for safety).

- The project is for the purpose of research and/or teaching. It may not be of direct benefit to me.

- The privacy of the information I provide will be safeguarded. However should information of a private nature need to be disclosed for moral, clinical or legal reasons, I will be given an opportunity to negotiate the terms of this disclosure.

- The security of the research data is assured during and after completion of the study. The data collected during the study may be published, and a report of the project outcomes will be provided to Mr Dinesh K. Kumar and published in my thesis. Any information that will identify me will not be used.

\section{Participant's Consent}

Participant Name

Participant Signature

Date

Witness Name

Witness Signature

Date

Participants should be given a photocopy of this consent form after it has been signed.

Any complaints about your participation in this project may be directed to the Secretary, RMIT Human Research Ethics Committee, University Secretariat, RMIT, GPO Box 2476V, Melbourne, 3001. The telephone number is (03) 9925 1745. Details of the complaints procedure are available from the above address. 
[1] B. Detenber, R. Simons, and J. Reiss, "The emotional significance of color in television presentations," Media Psychology, vol. 2, pp. 331-335, 2000.

[2] R. Levenson, P. Ekman, K. Heider, and W. Friesen, "Emotion and autonomic nervous system activity in the minangkabau of west sumatra," Journal of Personality and Social Psychology, vol. 64, pp. 927-988, 1992.

[3] S. Vrana, "The psychology of disgust: differentiating negative emotional contexts with facial emg," Psychology, vol. 30, pp. 927-988, 1993.

[4] S. Vrana, B. Cuthbert, and P. Lang, "Fear imagery and text processing," Psychology, vol. 23, pp. 247-253, May 1986.

[5] C. Smith, "Dimensions of appraisal and physiological response in emotion," Journal of Personality and Social Psychology, vol. 56, 339-353.

[6] R. Lane, P. Chua, and R. Dolan, "Common effects of emotional valence, arousal and attention on neural activation during visual processing of picture," Neuropsychologia, vol. 37, pp. 989997, 1999.

[7] H. Storm, K. Myre, M. Rostrup, O. Stokland, M. Lien, and J. R_der, "Skin conductance correlates with perioperative stress," Acta Anaesthesiologica Scandinavica, vol. 46, pp. 887895, 2002. 
[8] J. Healey, J. Seger, and R. Picard, "Quantifying driver stress: Developing a system for collecting and processing bio-metric signals in natural situations," Proceedings of the Rocky Mountian Bio-Engineering Symposium, 1999.

[9] S. Heo, N.and Sundar, "Emotional responses to web advertising: The effects of animation, position, and product involvement on physiological arousal," Advertising division at the annual conference of the Association for Education in Journalism and Mass Communication (AEJMC), Phoenix Arizona, 2000.

[10] J. Kaufman and J. Christensen, IES Lighting Handbook (Appilication volume). New York: New York, 1987.

[11] I. Flagge, 1994 Annual of Light and Architecture. Berlin: Ernst \& Sohn, 1994.

[12] J. Turner, Lighting: An introduction to Light, Lighting and Light Use. Batsford:Elsevier Science, 1994.

[13] K. Frasca-Bealieu, "Interior design for ambulatory care: How to reduce stress and anxiety of patients and families," Ambulatory care management Gaithersburg, vol. 22, pp. 67-73, 1999.

[14] I. Knez and I. Enmarker, "Effect of office lighting on mood and cognitive performance and a gender effect in work-related judgment," Envinronment and behaviour, vol. 30, pp. 553567, July 1998.

[15] W. Hathaway, "School performance: A case of daylight robbery," Psychology today, vol. 27, p. 8, 1992.

[16] V. Kiernan, “Where there's light there's brass," New Scientist, vol. 144, p. 8, December 1994.

[17] M. Shepard, "Lighting and the human condition," EPRI Journal, vol. 11, no. 9, pp. 16-23, December 1986.

[18] G. H. Taylor, "Office lighting and its effect on good employee performance," The Office, vol. 91, pp. 91-94, May 1980. 
[19] J. A. Veitch and G. R. Newsham, "Quantifying lighting quality based on experimental investigations of end user performance and preference," In Proceedings of Right Light Three, The Third European Conference on Energy-Efficient Lighting, Newcastle-upon-Tyne, England, vol. 1, pp. 119-127, June 1995.

[20] P. Lang, M. Greenwald, M. Bradley, and A. Hamm, "Looking at pictures: affective, facial, visceral, and behavioural reactions," Psychophysiology, vol. 30, pp. 261-273, 1993.

[21] M. Bradley and P. Lang, Measuring Emotion: Behavior, feeling and physiology, R. Lane, L. Nadel, G. Ahern, J. Allen, A. Kaszniak, S. Rapcsak, and G. Schwartz, Eds. New York: New York : Oxford University Press, 2000.

[22] D. K. Tiller, "Towards a deeper understanding of psychological effects of lighting," Journal of the Illuminating Engineering Society, vol. 19, no. 2, pp. 59-65, 1990.

[23] J. A. Veitch and G. R. Newsham, "Determinants of lighting quality i: State of the science," Paper presented at the 1996 Annual Conference of the Illuminating Engineering Society of North America, Cleveland, OH, August 1996.

[24] P. Valdez and A. Mehrabian, "Effects of color on emotion," Journal of Experimental Psychology, vol. 123, no. 4, pp. 394-409, 1994.

[25] F. Birren, Light, Color and Environment. Schiffer Publishing, 1988.

[26] A. Durak, "Effect of artificial lighting on perception of interior spaces," Interior Architecture \& Environmental Design 501 Graduate Studio - Commentary Bibliography Series , www.art.bilkent.edu.tr/iaed/cb/Odemis.html, January 1997.

[27] T. Gohara, H. Mizuta, I. Takeuchi, O. Tsuda, K. Yana, T. Yanai, Y. Yamamoto, and N. Kishi, "Heart rate variability change induced by the mental stress: the effect of accumulated fatigue," in Biomedical Engineering Conference, 1996., Proceedings of the 1996 Fifteenth Southern, 1996, pp. 367-369.

[28] J. Sims, D. Vashishtha, P. Rani, R. Brackin, and N. Sarkar, "Stress detection for implicit humanrobot co-operation," in Proc. 5th Biannual World Automation Congress, vol. 14, 2002, pp. $567-572$. 
[29] H. Steiner, E. Ryst, J. Berkowitz, M. A. Gschwendt, and C. Koopman, "Boys and girls responses to stress: affect and heart rate during a speech task," Journal of Adolescent Health, vol. 30, no. 4, Supplement 1, pp. 14-21, 2002.

[30] W. D. McArdle, F. I. Katch, and V. L. Katch, Essentials of Exercise Physiology. Philadelphia: Lea \& Febiger, 1994.

[31] H. Steenis, W. Martens, and J. Tulen, "Time-frequency parameters of heart-rate variability," IEEE Engineering in Medicine and Biology, vol. 21, no. 4, pp. 46-58, 2002.

[32] M. Tarvainen, P. Karjalainen, A. Koistinen, and M. Valkonen-Korhonen, "Principal component analysis of galvanic skin responses," Proceedings of the 22nd Annual International Conference of the IEEE, Chicago, vol. 4, pp. 3011-3014, 2000.

[33] P. Averty, S. Athenes, C. Collet, and A. Dittmar, "Evaluating a new index of mental workload in real atc situation using psychophysiological measures," in Digital Avionics Systems Conference, 2002. Proceedings. The 21st, vol. 2, 2002, pp. 7A4-1-7A4-13 vol.2.

[34] J. Healey and R. W. Picard, "Startlecam: A cybernetic wearable camera," in $I S W C, 1998, \mathrm{pp}$. $42-49$.

[35] J. Healey, J. Seger, and R. Picard, "Quantifying driver stress: Developing a system for collecting and processing bio-metric signals in natural situations," Biomedical Sciences Instrumentation, vol. 35, pp. 193-198, 1999.

[36] A. Mehrabian and J. Russell, "An approach to environmental psychology," vol. Cambridge, MA: MIT Press.

[37] M. Margaret, M. Bradley, and P. J. Lang, "Measurinf emotion: The self-assessment manikin and the semantic differential," Elsevier, vol. 25, no. 1, pp. 49-59, 1994.

[38] R. Hodes, E. Cook, and P. Lang, "Individual differences in autonomic response: conditioned association or conditioned fear," Psychophysiology, vol. 22, pp. 545-560, 1985.

[39] M. Greenwald, E. Cook, and P. Lang, "Affective judgement and psychophysiological response: dimensional covariation in the evaluation of pictorial stimuli," Journal of Psychophysiology, vol. 3, pp. 51-64, 1989. 
[40] P. Lang, M. Greenwald, M. Bradley, and A. Hamm, "Looking at pictures: evaluative, facial, visceral and behavioral responses," Psychophysiology, 1993.

[41] G. Miller, D. Levin, M. Kozak, E. Cook, A. McLean, and P. Lang, "Individual differences in emotional imagery," Cognition and Emotion, vol. 1, pp. 367-390, 1987.

[42] J. Morris, M. Bradley, C. Waine, and J. Lang, "Assessing affective reactions to advertisments using self-assessment manikin (sam)," Proceedings of the Southern Marketing Association, 1992.

[43] D. McNeil and D. G. Brunetti, "Pain and fear: a bioinformational perspective on responsivity to imagery," Behavior Research Therapy, vol. 30, pp. 513-520, 1992.

[44] F. Scheer, L. Doornen, and R. Buijs, "Light and diurnal cycle affect human heart rate: Possible role for the circadian pacemaker," Journal of Biological Rhythms, vol. 14, no. 3, pp. $202-$ 212, 1999.

[45] R. Leproult, E. Colecchia, M. L'Hermite-Baleriaux, and E. Van Cauter, "Transition from dim to bright light in the morning induces an immediate elevation of cortisol levels," Journal of Clinical Endocrinology 83 Metabolism, vol. 86, no. 1, pp. 151-157, 2000.

[46] J. Niskanen, M. Tarvainen, p. Ranta-aho, and P. Karjalainen, "Software for advanced hrv analysis," Computer Methods and Programs in Biomedicine, vol. 76, no. 1, pp. 73-81, 2002.

[47] I. Knez and C. Kers, "Effect of indoor lighting, gender and age on mood and cognitive performance," Environment and Behavior, vol. 32, no. 16, pp. 817-831, November 2000. 